\title{
Comparing geometric realizations of tricategories
}

\author{
ANTONIO M CEGARRA \\ BENJAMÍN A HEREDIA
}

\begin{abstract}
This paper contains some contributions to the study of classifying spaces for tricategories, with applications to the homotopy theory of monoidal categories, bicategories, braided monoidal categories and monoidal bicategories. Any small tricategory has various associated simplicial or pseudosimplicial objects and we explore the relationship between three of them: the pseudosimplicial bicategory (so-called Grothendieck nerve) of the tricategory, the simplicial bicategory termed its Segal nerve and the simplicial set called its Street geometric nerve. We prove that the geometric realizations of all of these 'nerves of the tricategory' are homotopy equivalent. By using Grothendieck nerves we state the precise form in which the process of taking classifying spaces transports tricategorical coherence to homotopy coherence. Segal nerves allow us to prove that, under natural requirements, the classifying space of a monoidal bicategory is, in a precise way, a loop space. With the use of geometric nerves, we obtain simplicial sets whose simplices have a pleasing geometrical description in terms of the cells of the tricategory and we prove that, via the classifying space construction, bicategorical groups are a convenient algebraic model for connected homotopy 3-types.
\end{abstract}

18D05, 55P15; 18D10, 55P35

\section{Introduction and summary}

The process of taking classifying spaces of categorical structures has shown its relevance as a tool in algebraic topology and algebraic $K$-theory. One of the main reasons for this is that classifying space constructions transport categorical coherence to homotopic coherence. We can easily stress the historical relevance of the construction of classifying spaces by recalling that Quillen [37] defines a higher algebraic $K$-theory by taking homotopy groups of the classifying spaces of certain categories. Joyal and Tierney [30] have shown that Gray groupoids are a suitable framework for studying homotopy 3-types. Monoidal categories were shown by Stasheff [41] to be algebraic models for loop spaces, and work by May [36] and Segal [39] showed that classifying spaces of symmetric monoidal categories provide the most noteworthy examples of spaces with the extra structure required to define an $\Omega$-spectrum, a fact exploited with great success in algebraic $K$-theory. 
This paper contributes to the study of classifying spaces for (small) tricategories, introduced by Gordon, Power and Street in [20]. Since our results find direct applications to monoidal categories, bicategories, braided monoidal categories, or monoidal bicategories, the paper will quite possibly be of special interest to $K$-theorists as well as to researchers interested in homotopy theory of higher categorical structures. This theory has demonstrated relevance as a tool for the treatment of an extensive list of subjects of recognized mathematical interest in several mathematical contexts beyond homotopy theory, such as algebraic geometry, geometric structures on low-dimensional manifolds, string field theory, or topological quantum theory and conformal field theory.

We explore the relationship amongst three different 'nerves' that might reasonably be associated to any tricategory $\mathcal{T}$. These are the pseudosimplicial bicategory called the Grothendieck nerve $\mathrm{N} \mathcal{T}: \Delta^{\mathrm{op}} \rightarrow$ Bicat, the simplicial bicategory termed the Segal nerve $\mathrm{ST}: \Delta^{\mathrm{op}} \rightarrow \mathbf{H o m}$, and the simplicial set called the geometric nerve of the tricategory $\Delta \mathcal{T}: \Delta^{\mathrm{op}} \rightarrow$ Set. Since, as we prove, these three nerve constructions lead to homotopy equivalent spaces, any one of these spaces could therefore be taken as the classifying space $\mathrm{B}_{3} \mathcal{T}$ of the tricategory. Many properties of the classifying space construction for tricategories, $\mathcal{T} \mapsto \mathrm{B}_{3} \mathcal{T}$, may be easier to establish depending on the nerve used for realizations. Here, both for historical reasons and for theoretical interest, it is appropriate to start with the Grothendieck nerve construction to introduce $\mathrm{B}_{3} \mathcal{T}$. Let us briefly recall that it was Grothendieck who first associated a simplicial set $\mathrm{NC}$ to a small category $C$, calling it its nerve. Its $p$-simplices are composable $p$-tuples $x_{0} \rightarrow \cdots \rightarrow x_{p}$ of morphisms in $C$. A geometric realization of the nerve is the classifying space of the category, $\mathrm{BC}=|\mathrm{NC}|$. A first result in this paper shows how the Grothendieck nerve construction for categories rises to tricategories. Thus, we prove the following.

Theorem 3.1 Any tricategory $\mathcal{T}$ defines a pseudosimplicial bicategory, that is, a trihomomorphism $\mathrm{N} \mathcal{T}=(\mathrm{N} \mathcal{T}, \chi, \omega): \Delta^{\mathrm{op}} \rightarrow$ Bicat, whose bicategory of $p$-simplices

$$
\mathrm{N}_{p} \mathcal{T}=\bigsqcup_{\left(x_{0}, \ldots, x_{p}\right) \in \mathrm{Ob} \mathcal{T}^{p+1}} \mathcal{T}\left(x_{p-1}, x_{p}\right) \times \mathcal{T}\left(x_{p-2}, x_{p-1}\right) \times \cdots \times \mathcal{T}\left(x_{0}, x_{1}\right)
$$

consists of $p$-tuples of horizontally composable cells.

Then, heavily dependent on the results by Carrasco, Cegarra and Garzón in [12], where an analysis of classifying spaces is performed for lax diagrams of bicategories following the way Segal [39] and Thomason [47] analyzed lax diagrams of categories, we introduce the classifying space $\mathrm{B}_{3} \mathcal{T}$, of a tricategory $\mathcal{T}$, to be the classifying space of its Grothendieck nerve NT . Briefly, say that the so-called Grothendieck construction [12, Section 3.1] on the pseudosimplicial bicategory $\mathrm{N} \mathcal{T}$ produces a 
bicategory $\int_{\Delta} \mathrm{N} \mathcal{T}$. Again, the Grothendieck nerve construction on this bicategory $\int_{\Delta} \mathrm{N} \mathcal{T}$ now gives rise to a normal pseudosimplicial category $\mathrm{N}\left(\int_{\Delta} \mathrm{N} \mathcal{T}\right): \Delta^{\mathrm{op}} \rightarrow$ Cat, on which one more Grothendieck construction leads to a category $\int_{\Delta} \mathrm{N}\left(\int_{\Delta} \mathrm{N} \mathcal{T}\right)$, whose classifying space is, by definition, the classifying space of the tricategory, that is

$$
\mathrm{B}_{3} \mathcal{T}=\left|\mathrm{N}\left(\int_{\Delta} \mathrm{N}\left(\int_{\Delta} \mathrm{N} \mathcal{T}\right)\right)\right|
$$

or, in other words, $\mathrm{B}_{3} \mathcal{T}=\mathrm{B}_{2} \int_{\Delta} \mathrm{N} \mathcal{T}=\mathrm{B} \int_{\Delta} \mathrm{N}\left(\int_{\Delta} \mathrm{N} \mathcal{T}\right)$, where $\mathrm{B}_{2} \mathcal{B}$ denotes the classifying space of any bicategory $\mathcal{B}$ as defined by Carrasco, Cegarra and Garzón [11, Definition 3.1]. The behavior of this classifying space construction, $\mathcal{T} \mapsto \mathrm{B}_{3} \mathcal{T}$, can be summarized as follows (see Propositions 3.2 and 5.6 and 5.7):

- Any trihomomorphism $F: \mathcal{T} \rightarrow \mathcal{T}^{\prime}$ induces a continuous map $\mathrm{B}_{3} F: \mathrm{B}_{3} \mathcal{T} \rightarrow \mathrm{B}_{3} \mathcal{T}^{\prime}$.

- For any composable trihomomorphisms $F: \mathcal{T} \rightarrow \mathcal{T}^{\prime}$ and $F^{\prime}: \mathcal{T}^{\prime} \rightarrow \mathcal{T}^{\prime \prime}$, there is a homotopy $\mathrm{B}_{3} F^{\prime} \mathrm{B}_{3} F \simeq \mathrm{B}_{3}\left(F^{\prime} F\right): \mathrm{B}_{3} \mathcal{T} \rightarrow \mathrm{B}_{3} \mathcal{T}^{\prime \prime}$ and for any tricategory $\mathcal{T}$, there is a homotopy $\mathrm{B}_{3} 1_{\mathcal{T}} \simeq 1_{\mathrm{B}_{3} \mathcal{T}}$.

- If $F, G: \mathcal{T} \rightarrow \mathcal{T}^{\prime}$ are two trihomomorphisms, then any tritransformation $F \Rightarrow G$ canonically defines a homotopy $\mathrm{B}_{3} F \simeq \mathrm{B}_{3} G: \mathrm{B}_{3} \mathcal{T} \rightarrow \mathrm{B}_{3} \mathcal{T}^{\prime}$ between the induced maps on classifying spaces.

- Any triequivalence of tricategories $\mathcal{T} \rightarrow \mathcal{T}^{\prime}$ induces a homotopy equivalence on classifying spaces $\mathrm{B}_{3} \mathcal{T} \simeq \mathrm{B}_{3} \mathcal{T}^{\prime}$.

For instance, for every tricategory $\mathcal{T}$ there is a Gray-category $\mathrm{G}(\mathcal{T})$ with a triequivalence $\mathcal{T} \rightarrow \mathrm{G}(\mathcal{T})$, thanks to the coherence theorem for tricategories by Gordon, Power and Street [20, Theorem 8.1]. Then we have:

There is an induced homotopy equivalence $\mathrm{B}_{3} \mathcal{T} \simeq \mathrm{B}_{3} \mathrm{G}(\mathcal{T})$.

To deal with the delooping properties of certain classifying spaces we introduce, for any tricategory $\mathcal{T}$, its Segal nerve $\mathrm{S} \mathcal{T}$. This is a simplicial bicategory whose bicategory of $p$-simplices, $\mathrm{S}_{p} \mathcal{T}$, is the bicategory of unitary trihomomorphisms of the ordinal category $[p]$ into $\mathcal{T}$. Each $S \mathcal{T}$ is a special simplicial bicategory, in the sense that the Segal projection homomorphisms on it are biequivalences of bicategories, and thus it is a weak 3-category from the standpoint of Tamsamani [46] and Simpson [40]. When $\mathcal{T}$ is a reduced tricategory (ie with only one object), then the simplicial space $\mathrm{B}_{2} \mathrm{~S} \mathcal{T}: \Delta^{\mathrm{op}} \rightarrow$ Top, obtained by replacing the bicategories $\mathrm{S}_{p} \mathcal{T}$ by their classifying spaces $\mathrm{B}_{2} \mathrm{~S}_{p} \mathcal{T}$, is a special simplicial space. Therefore, according to Segal [39, Proposition 1.5], under favorable circumstances, $\Omega\left|\mathrm{B}_{2} \mathrm{~S} \mathcal{T}\right|$ is homotopy equivalent to $\mathrm{B}_{2} \mathrm{~S}_{1} \mathcal{T}$. In our development here, the relevant result is the following.

Theorem 4.14 For any tricategory $\mathcal{T}$, there is a homotopy equivalence $\mathrm{B}_{3} \mathcal{T} \simeq\left|\mathrm{B}_{2} \mathrm{~S} \mathcal{T}\right|$. 
Any monoidal bicategory $(\mathcal{B}, \otimes)$ gives rise to a one-object tricategory $\Sigma(\mathcal{B}, \otimes)$, its suspension tricategory following Street's terminology [44, Section 9, Example 2] or delooping in the terminology of Kapranov and Voevodsky [31] or Berger [5]. Defining the classifying space of a monoidal bicategory $(\mathcal{B}, \otimes)$ to be the classifying space of its suspension tricategory, that is, $\mathrm{B}_{3}(\mathcal{B}, \otimes)=\mathrm{B}_{3} \Sigma(\mathcal{B}, \otimes)$, we prove the following extension to bicategories of the aforementioned fact by Stasheff on monoidal categories.

Theorem 4.17 Let $(\mathcal{B}, \otimes)$ be a monoidal bicategory such that, for any object $x \in \mathcal{B}$, the homomorphism $x \otimes-: \mathcal{B} \rightarrow \mathcal{B}$ induces a homotopy autoequivalence on the classifying space $\mathrm{B}_{2} \mathcal{B}$ of $\mathcal{B}$. Then there is a homotopy equivalence

$$
\mathrm{B}_{2} \mathcal{B} \simeq \Omega \mathrm{B}_{3}(\mathcal{B}, \otimes)
$$

between the classifying space of the underlying bicategory and the loop space of the classifying space of the monoidal bicategory.

If $(C, \otimes, c)$ is any braided monoidal category, then, thanks to the braiding, the suspension of the underlying monoidal category $\Sigma(C, \otimes)$, which is actually a bicategory, has the structure of a monoidal bicategory. Hence the double suspension tricategory $\Sigma^{2}(C, \otimes, c)$ is defined. According to Jardine [28, Section 3], the classifying space of the monoidal category $\mathrm{B}_{2}(C, \otimes)$ is the classifying space of its suspension bicategory and, following Carrasco, Cegarra and Garzón [12, Definition 6.1], the classifying space of the braided monoidal category $\mathrm{B}_{3}(C, \otimes, c)$ is the classifying space of its double suspension tricategory. Hence from the above result we get the following.

Corollary 4.20 (i) For any braided monoidal category $(C, \otimes, c)$ there is a homotopy equivalence

$$
\mathrm{B}_{2}(C, \otimes) \simeq \Omega \mathrm{B}_{3}(C, \otimes, c) .
$$

(ii) Let $(C, \otimes, c)$ be a braided monoidal category such that, for any object $x \in C$, the functor $x \otimes-: C \rightarrow C$ induces a homotopy autoequivalence on the classifying space of $C$. Then there is a homotopy equivalence

$$
\mathrm{B} C \simeq \Omega^{2} \mathrm{~B}_{3}(C, \otimes, c) .
$$

Thus, under natural hypotheses, the double suspension tricategory $\Sigma^{2}(C, \otimes, c)$ is a categorical model for the double delooping space of the classifying space of the underlying category $C$, a fact already proved in [12, Theorem 6.10] (cf Berger [5, Proposition 2.11] and Baltenau, Fiedorowicz, Schwänzl and Vogt [3, Theorem 2.2]). 
The process followed for defining the classifying space of a tricategory $\mathcal{T}$ by means of its Grothendieck nerve $\mathrm{N} \mathcal{T}$ is quite indirect and the $\mathrm{CW}$-complex $\mathrm{B}_{3} \mathcal{T}$ thus obtained has little apparent intuitive connection with the cells of the original tricategory. However, when $\mathcal{T}$ is a (strict) 3-category, then the space $|\operatorname{diag} \mathrm{NNN} \mathcal{T}|$, the geometric realization of the simplicial set diagonal of the 3-simplicial set 3-fold nerve of $\mathcal{T}$, has usually been taken as the 'correct' classifying space of the 3-category. In Example 4.11, we state that for a 3 -category $\mathcal{T}$, there is a homotopy equivalence

$$
\mathrm{B}_{3} \mathcal{T} \simeq|\operatorname{diag} \mathrm{NNN} \mathcal{T}| .
$$

The construction of the simplicial set diag NNN $\mathcal{T}$ for 3-categories does not work in the nonstrict case since the compositions in arbitrary tricategories are not associative and not unitary, which is crucial for the 3-simplicial structure of the triple nerve NNN $\mathcal{T}$, but only up to coherent equivalences or isomorphisms. There is, however, another convincing way of associating a simplicial set to a 3-category $\mathcal{T}$ through its geometric nerve $\Delta \mathcal{T}$, thanks to Street [43]. He extends each ordinal $[p]=\{0<1<\cdots<p\}$ to a $p$-category $\mathcal{O}_{p}$, the $p^{\text {th }}$-oriental, such that the $p$-simplices of $\Delta \mathcal{T}$ are just the 3 -functors $\mathcal{O}_{p} \rightarrow \mathcal{T}$. Thus, $\Delta \mathcal{T}$ is a simplicial set whose 0 -simplices are the objects ( 0 -cells) $F 0$ of $\mathcal{T}$, whose 1 -simplices are the 1 -cells $F_{0,1}: F 0 \rightarrow F 1$, whose 2-simplices

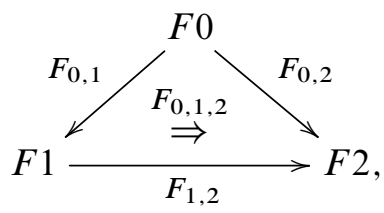

consist of two composable 1 -cells and a $2-$ cell $F_{0,1,2}: F_{1,2} \otimes F_{0,1} \Rightarrow F_{0,2}$ and so on. In fact, the geometric nerve construction $\Delta \mathcal{T}$ even works for arbitrary tricategories $\mathcal{T}$, as Duskin [16] and Street [45] pointed out, and we discuss here in detail. The geometric nerve $\Delta \mathcal{T}$ is defined to be the simplicial set whose $p$-simplices are unitary lax functors of the ordinal category $[p]$ in the tricategory $\mathcal{T}$. This is a simplicial set which completely encodes all the structure of the tricategory and, furthermore, the cells of its geometric realization $|\Delta \mathcal{T}|$ have a pleasing geometrical description in terms of the cells of $\mathcal{T}$. As a main result in the paper, we state and prove the following.

Theorem 5.4 For any tricategory $\mathcal{T}$, there is a homotopy equivalence $\mathrm{B}_{3} \mathcal{T} \simeq|\Delta \mathcal{T}|$.

If $(\mathcal{B}, \otimes)$ is any monoidal bicategory, then its geometric nerve, $\Delta(\mathcal{B}, \otimes)$, is defined to be the geometric nerve of its suspension tricategory $\Sigma(\mathcal{B}, \otimes)$. Then, in Example 5.11, we obtain that there is a homotopy equivalence

$$
\mathrm{B}_{3}(\mathcal{B}, \otimes) \simeq|\Delta(\mathcal{B}, \otimes)| .
$$


For instance, since the geometric nerve of a braided monoidal category $(C, \otimes, c)$ is the geometric nerve of its double suspension tricategory, ie $\Delta(C, \otimes, c)=\Delta \Sigma^{2}(C, \otimes, c)$, the existence of a homotopy equivalence

$$
\mathrm{B}_{3}(C, \otimes, c) \simeq|\Delta(C, \otimes, c)|
$$

follows, a fact proved by Carrasco, Cegarra and Garzón [12, Theorem 6.9].

The geometric nerve $\Delta(\mathcal{B}, \otimes)$ of any given monoidal bicategory $(\mathcal{B}, \otimes)$ is a $\operatorname{Kan}$ complex if and only if $(\mathcal{B}, \otimes)$ is a bicategorical group, that is, a monoidal bicategory whose 2-cells are isomorphisms, whose 1-cells are equivalences and each object $x$ has a quasi-inverse with respect to the tensor product. In other words, a bicategorical group is a monoidal bicategory whose suspension tricategory $\Sigma(\mathcal{B}, \otimes)$ is a trigroupoid (or Azumaya tricategory in the terminology of Gordon, Power and Street [20]). The geometric nerve of any bicategorical group $(\mathcal{B}, \otimes)$ is then a Kan complex, whose classifying space $\mathrm{B}_{3}(\mathcal{B}, \otimes)$ is a path-connected homotopy 3-type. In fact, every connected homotopy 3-type can be realized in this way from a bicategorical group, as suggested by the unpublished but widely publicized result of Joyal and Tierney [30] that Gray-groups (called semistrict 3-groups by Baez and Neuchl [2]) model connected homotopy 3-types (see also Berger [5], Lack [33], or Leroy [35]). Recall that by the coherence theorem for tricategories, every bicategorical group is monoidal biequivalent to a Gray-group. In Section 5.1, we outline in some detail the proof of the following statement.

Proposition 5.12 For any path-connected pointed $C W$-complex $X$, there is a bicategorical group $(\mathcal{B}(X), \otimes)$ with a homotopy equivalence $\mathrm{B}_{3}(\mathcal{B}(X), \otimes) \simeq X$ if and only if $\pi_{i} X=0$ for $i \geq 4$.

The bicategorical group $(\mathcal{B}(X), \otimes)$ we build, associated to any space $X$ as above, might be recognized as a skeleton of Gurski's monoidal fundamental bigroupoid of the loop space of $X,\left(\Pi_{2}(\Omega X), \otimes\right)$ [23, Theorem 1.4]. In the particular case when in addition $\pi_{3} X=0$, the resulting bicategorical group $(\mathcal{B}(X), \otimes)$ has all its 2-cells identities, so it is actually a categorical group in the sense of Joyal and Street [29, Definition 3.1]. In the particular case where $\pi_{1} X=0$, the bicategorical group $(\mathcal{B}(X), \otimes)$ has only one object, so that it is the suspension of a braided categorical group; see Cheng and Gurski [15, Section 2]. Hence, our proof implicitly covers two relevant particular cases, already well known from Joyal and Tierney [30]; see also Carrasco and Cegarra [9, Theorems 2.6 and 2.10]. One case states that categorical groups are a convenient algebraic model for connected homotopy 2-types. The other case states that braided categorical groups are algebraic models for connected, simply connected homotopy 3-types. 


\subsection{The organization of the paper}

The paper is organized in five sections. Section 2 is quite technical but crucial to our discussions. It is dedicated to establishing some results concerning the notion of a lax functor from a category into a tricategory, which is at the heart of several constructions of nerves for tricategories used in the paper. In Section 3, we mainly include the construction of the Grothendieck nerve $\mathrm{N} \mathcal{T}: \Delta^{\mathrm{op}} \rightarrow$ Bicat for any tricategory $\mathcal{T}$, and the study of the basic properties of the Grothendieck nerve construction $\mathcal{T} \mapsto \mathrm{N} \mathcal{T}$ with respect to trihomomorphisms of tricategories. Section 4 contains the definition of the classifying space $\mathrm{B}_{3} \mathcal{T}$ of a tricategory $\mathcal{T}$. The main facts concerning the classifying space construction $\mathcal{T} \mapsto \mathrm{B}_{3} \mathcal{T}$ are established here. In this section we also study the relationship between $\mathrm{B}_{3} \mathcal{T}$ and the space realization of the Segal nerve of a tricategory, $\mathrm{ST}: \Delta^{\mathrm{op}} \rightarrow \mathbf{H o m}$, which, for instance, we apply to show how the classifying space of any monoidal bicategory realizes a delooping space. Section 5 is mainly dedicated to describing the geometric nerve $\Delta \mathcal{T}: \Delta$ op $\rightarrow$ Set of a tricategory $\mathcal{T}$ and to proving the existence of homotopy equivalences $\mathrm{B}_{3} \mathcal{T} \simeq|\Delta \mathcal{T}|$. Also, by means of the geometric nerve construction for monoidal bicategories, we show there that bicategorical groups are a convenient algebraic model for connected homotopy 3-types. And finally, the Appendix collects the various coherence conditions used throughout the paper and the proofs of lemmas in the preparatory Section 2.

\subsection{Notation}

We refer to Benabou [4] and Street [44] for background on bicategories. For any bicategory $\mathcal{B}$, the composition in each hom category $\mathcal{B}(x, y)$, that is, the vertical composition of 2-cells, is denoted by $v \cdot u$, while the symbol $\circ$ is used to denote the horizontal composition functors $\circ: \mathcal{B}(y, z) \times \mathcal{B}(x, y) \rightarrow \mathcal{B}(x, z)$. The identity of an object is written as $1_{x}: x \rightarrow x$ and we shall use the letters $\boldsymbol{a}, \boldsymbol{r}$ and $\boldsymbol{l}$ to denote the associativity, right unit and left unit constraints of the bicategory, respectively.

In this paper we use the notion of tricategory $\mathcal{T}=(\mathcal{T}, \boldsymbol{a}, \boldsymbol{l}, \boldsymbol{r}, \pi, \mu, \lambda, \rho)$ as it was introduced by Gordon, Power and Street in [20] but with a minor alteration: we require that the homomorphisms of bicategories picking out units are normalized, and then written simply as $1_{t} \in \mathcal{T}(t, t)$. This restriction is not substantive (see Gurski [25, Theorem 7.24]) but it does slightly reduce the amount of coherence data we have to deal with. For any object $t$ of the tricategory $\mathcal{T}$, the arrow $\boldsymbol{r} 1_{t}: 1_{t} \rightarrow 1_{t} \otimes 1_{t}$ is an equivalence in the hom bicategory $\mathcal{T}(t, t)$, with the arrow $l 1_{t}: 1_{t} \otimes 1_{t} \rightarrow 1_{t}$ an adjoint quasi-inverse; see [25, Lemma 7.7]. Hereafter, we suppose the adjoint quasi-inverse of $\boldsymbol{r}, \boldsymbol{r}^{\bullet} \dashv \boldsymbol{r}$, has been chosen such that $\boldsymbol{r}^{\bullet} 1_{t}=\boldsymbol{l} 1_{t}$, with the isomorphism $\boldsymbol{r}^{\bullet} 1_{t} \cong \boldsymbol{l} 1_{t}$ being an identity. We will extensively use the coherence results from [25, Corollaries 10.6, 10.15], particulary the following facts easily deduced from them. 
Fact 1.1 Any two pasting diagrams in a tricategory $\mathcal{T}$ with the same source and target constructed out of constraint $2-$ and 3 -cells of $\mathcal{T}$ are equal.

Fact 1.2 Given a trihomomorphism $H: \mathcal{T} \rightarrow \mathcal{T}^{\prime}$, any two pasting diagrams in $\mathcal{T}^{\prime}$ with the same source and target constructed out of constraint 2- and 3-cells of $\mathcal{T}, \mathcal{T}^{\prime}$ and $H$ are equal.

The category of bicategories with homomorphisms (or pseudofunctors) between them will be denoted by Hom and Bicat will denote the tricategory of small bicategories, homomorphisms, pseudotransformations and modifications. Thus we follow the notation by Gordon, Power and Street [20, Notation 4.9 and Section 5] and Gurski [25, Section 5.1]. If $F, F^{\prime}: \mathcal{B} \rightarrow \mathcal{C}$ are lax functors between bicategories, then we follow the convention of [20] in what is meant by a lax transformation $\alpha: F \Rightarrow F^{\prime}$. Hence, $\alpha$ consists of morphisms $\alpha x: F x \rightarrow F^{\prime} x$ and of 2-cells $\alpha_{a}: \alpha y \circ F a \Rightarrow F^{\prime} a \circ \alpha x$, subject to the usual axioms. In the structure of Bicat we use, the composition of pseudotransformations is taken to be

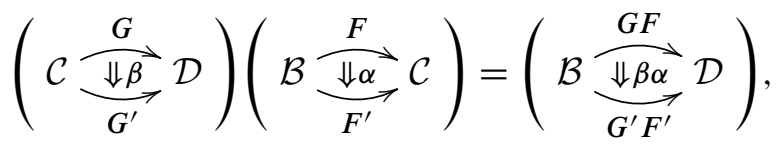

where $\beta \alpha=\beta F^{\prime} \circ G \alpha: G F \Rightarrow G^{\prime} F \Rightarrow G^{\prime} F^{\prime}$. Note the existence of the useful invertible modification

$$
\begin{gathered}
G F_{G \alpha} \stackrel{\beta F}{\Longrightarrow} G^{\prime} F \\
\Downarrow \stackrel{\beta F^{\prime}}{\Longrightarrow} \|_{G^{\prime} \alpha}^{\prime} F^{\prime}
\end{gathered}
$$

whose component at an object $x$ of $\mathcal{B}$ is $\beta_{\alpha x}$, the component of $\beta$ at the morphism $\alpha x$. The following fact will be also very useful.

Fact 1.3 Let $\alpha: F \Rightarrow F^{\prime}: \mathcal{B} \rightarrow \mathcal{C}$ be a lax transformation between homomorphisms of bicategories. Then for any $2-$ cell in $\mathcal{B}$

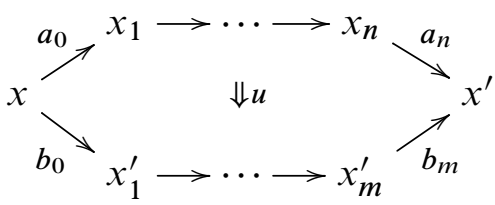


the following equality holds:

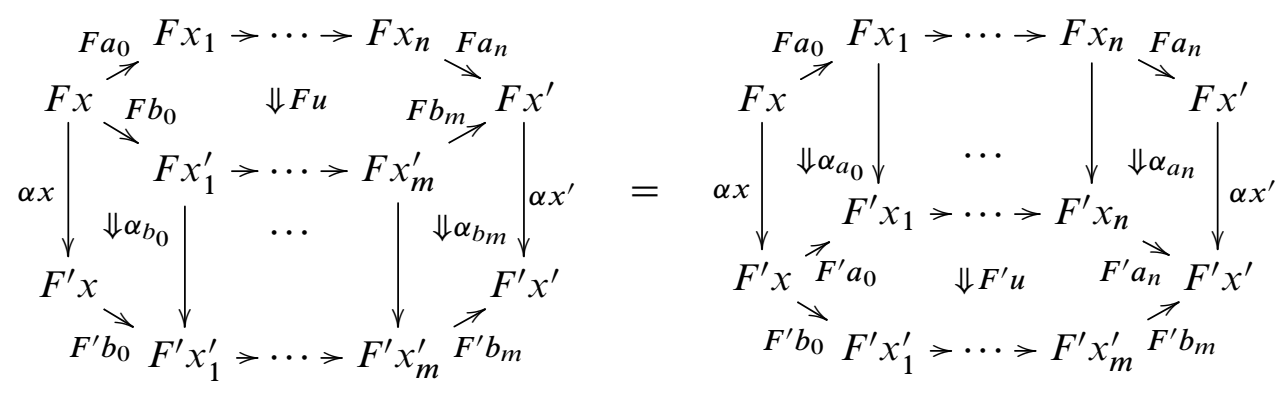

For general background on simplicial sets, we refer to the book by Goerss and Jardine [19]. The simplicial category is denoted by $\Delta$ and its objects, that is, the ordered sets $[n]=\{0,1, \ldots, n\}$, are usually considered as categories with only one morphism $(i, j): i \rightarrow j$ when $0 \leq i \leq j \leq n$. Then a nondecreasing map $[n] \rightarrow[m]$ is the same as a functor, so that we see $\Delta$, the simplicial category of finite ordinal numbers, as a full subcategory of Cat, the category (actually the 2-category) of small categories. Throughout the paper, Segal's geometric realization [38] of a simplicial (compactly generated topological) space $X: \Delta^{\mathrm{op}} \rightarrow$ Top is denoted by $|X|$. By regarding a set as a discrete space, the (Milnor) geometric realization of a simplicial set $X: \Delta^{\text {op }} \rightarrow$ Set is $|X|$. Following Quillen [37], the classifying space of a category $C$ is denoted by $\mathrm{B} C$.

\section{Lax functors from categories into tricategories}

As we will show in the paper, the classifying space of any tricategory can be realized up to homotopy by a simplicial set $\Delta \mathcal{T}$, whose $p$-simplices $\Delta[p] \rightarrow \Delta \mathcal{T}$ are lax functors $[p] \rightarrow \mathcal{T}$, where $[p]$ is regarded as tricategory in which the 2-cells and 3-cells are all identities, satisfying various requirements of normality. To be more precise, we recall the following.

A lax functor $F: I \rightarrow \mathcal{T}$, from a category $I$ to a tricategory $\mathcal{T}$, is the following data:

- For each object $i$ in $I$, an object $F i \in \mathrm{Ob} \mathcal{T}$

- For each arrow $a: i \rightarrow j$ in $I$, a $1-$ cell $F a: F i \rightarrow F j$

- For each pair of composable arrows

$$
i \stackrel{b}{\longrightarrow} j \stackrel{a}{\longrightarrow} k
$$

in $I$, a 2-cell $F_{a, b}: F a \otimes F b \Rightarrow F(a b)$ 
- For each object $i \in \mathrm{Ob} I$, a 2-cell $F_{i}: 1_{F i} \Rightarrow F 1_{i}$

- For any three composable arrows

$$
i \stackrel{c}{\longrightarrow} j \stackrel{b}{\longrightarrow} k \stackrel{a}{\longrightarrow} l
$$

in $I$, a $3-$ cell

$$
\begin{array}{cc}
(F a \otimes F b) \otimes F c \underset{a}{\stackrel{F_{a, b, c}}{\Rightarrow}} & F a \otimes(F b \otimes F c) \\
F_{a, b} \otimes 1 \Downarrow \underset{F_{a b, c}}{\rightleftharpoons} F(a b c) \underset{F_{a, b c}}{\leftrightarrows} F a \otimes F(b c) & \| 1 \otimes F_{b, c}
\end{array}
$$

- For any arrow $i \stackrel{a}{\longrightarrow} j$ in the index category $I$, two 3-cells

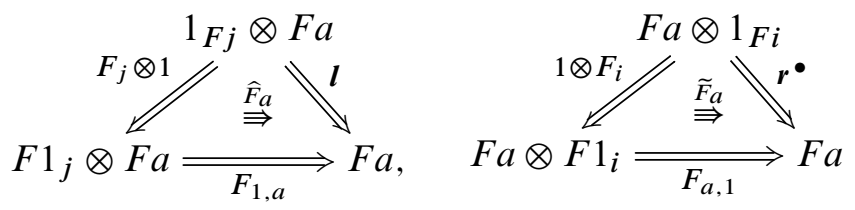

This data is required to satisfy the coherence conditions (CR1), (CR2) and (CR3) as stated in the Appendix.

Notice that we use a weaker notion of lax functor from that by Garner and Gurski in [18], where it is required for the structure 3-cells to be invertible. Furthermore, here the hom functors $I(i, j) \rightarrow \mathcal{T}(F i, F j)$ are normal.

The set of lax functors from a small category $I$ to a small tricategory $\mathcal{T}$ is denoted by

$$
\operatorname{Lax}(I, \mathcal{T}) \text {. }
$$

A lax functor $F: I \rightarrow \mathcal{T}$ is termed unitary or normal whenever the following conditions hold: for each object $i$ of $I, F 1_{i}=1_{F i}$ and $F_{i}=1_{1_{F i}}$; for each arrow $a: i \rightarrow j$ of $I$, $F_{a, 1_{i}}=r^{\bullet}: F a \otimes 1 \Rightarrow F a, F_{1_{j}, a}=l: 1 \otimes F a \Rightarrow F a$, and the 3-cells $F_{1, b, c}, F_{a, 1, c}$, $F_{a, b, 1}, \widehat{F}_{a}$ and $\widetilde{F}_{a}$ are the unique coherence isomorphisms. Furthermore, a lax functor $F: I \rightarrow \mathcal{T}$ whose structure 2-cells $F_{a, b}$ are all equivalences (in the corresponding hom bicategories of $\mathcal{T}$ where they lie) and whose structure 3-cells $F_{a, b, c}, \widehat{F}_{a}$ and $\widetilde{F}_{a}$, are all invertible is called homomorphic (or a trihomomorphism). The subsets of $\operatorname{Lax}(I, \mathcal{T})$ whose elements are the unitary, homomorphic and unitary homomorphic lax functors, are denoted respectively by

$$
\operatorname{Lax}_{\mathrm{u}}(I, \mathcal{T}), \quad \operatorname{Lax}_{\mathrm{h}}(I, \mathcal{T}), \quad \operatorname{Lax}_{\mathrm{uh}}(I, \mathcal{T})
$$


Example 2.1 Let $A$ be an abelian group and let $\Sigma^{2} A$ denote the tricategory (actually a 3-groupoid) having only one $i$-cell for $0 \leq i \leq 2$ and whose 3-cells are the elements of $A$, with all the compositions given by addition in $A$. Then, for any small category $I$ (eg a group $G$ or a monoid $M$ ), a unitary lax functor $F: I \rightarrow \Sigma^{2} A$ is the same as a function $F: \mathrm{N}_{3} I \rightarrow A$ satisfying the equation

$$
F(b, c, d)+F(a, b c, d)+F(a, b, c)=F(a b, c, d)+F(a, b, c d),
$$

such that $F(a, b, c)=0$ whenever any of the arrows $a, b$, or $c$ is an identity. Thus $\operatorname{Lax}_{\mathrm{u}}\left(I, \Sigma^{2} A\right)=Z^{3}(I, A)$, the set of normalized 3-cocycles of (the nerve $\mathrm{N} I$ of) the category $I$ with coefficients in the abelian group $A$.

\subsection{The bicategories $\operatorname{Lax}(I, \mathcal{T}), \operatorname{Lax}_{\mathrm{u}}(I, \mathcal{T}), \mathbb{L a x}_{\mathrm{h}}(I, \mathcal{T}), \operatorname{Lax}_{\mathrm{uh}}(I, \mathcal{T})$}

For any category $I$ and any tricategory $\mathcal{T}$, the set $\operatorname{Lax}(I, \mathcal{T})$ of lax functors from $I$ into $\mathcal{T}$ is the set of objects of a bicategory whose 1-cells are a kind of degenerated lax transformations between lax functors that agree on objects, called oplax icons by Garner and Gurski in [18]. When $\mathcal{T}=\mathcal{B}$ is a bicategory, that is, when the 3 -cells are all identities, these transformations have been considered by Bullejos and Cegarra in [7] under the name of relative to objects lax transformations, while they were termed icons by Lack in [32]. This bicategory, denoted by $\operatorname{Lax}(I, \mathcal{T})$, is as follows.

The cells of $\operatorname{Lax}(I, \mathcal{T})$ As we said above, lax functors $F: I \rightarrow \mathcal{T}$ are the 0 -cells of this bicategory. For any two lax functors $F, G: I \rightarrow \mathcal{T}$, a $1-$ cell $\Phi: F \Rightarrow G$ may exists only if $F$ and $G$ agree on objects, and is then given by specifying:

- For every arrow $a: i \rightarrow j$ in $I$, a 2-cell $\Phi a: F a \Rightarrow G a$ of $\mathcal{T}$

- For each pair of composable arrows

$$
i \stackrel{b}{\longrightarrow} j \stackrel{a}{\longrightarrow} k
$$

in $I$, a $3-$ cell

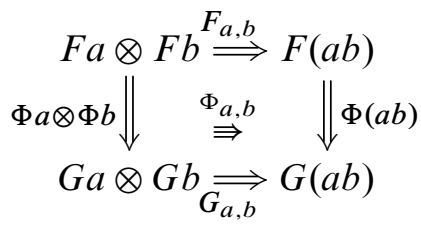

- For each object $i$ of the category $I$, a 3-cell

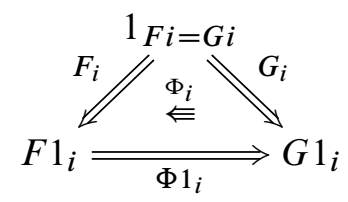


This data is subject to the axioms (CR4) and (CR5) as stated in the Appendix.

A 2-cell $M: \Phi \Rightarrow \Psi$, for two 1-cells $\Phi, \Psi: F \Rightarrow G$ in $\mathbb{L a x}(I, \mathcal{T})$, is an icon modification in the sense of [18], so it consists of a family of 3-cells in $\mathcal{T}, M a: \Phi a \Rightarrow \Psi a$, one for each arrow $a: i \rightarrow j$ in $I$, subject to the coherence condition (CR6).

Compositions in $\operatorname{Lax}(I, \mathcal{T})$ The vertical composition of a 2-cell $M: \Phi \Rightarrow \Psi$ with a 2-cell $N: \Psi \Rightarrow \Gamma$, for $\Phi, \Psi, \Gamma: F \Rightarrow G$, yields the 2 -cell $N \cdot M: \Phi \Rightarrow \Gamma$ which is defined using pointwise vertical composition in the hom bicategories of $\mathcal{T}$; that is, for each $a: i \rightarrow j$ in $I,(N \cdot M) a=(N a) \cdot(M a): \Phi a \Rightarrow \Gamma a: F a \Rightarrow G a$. The horizontal composition of 1-cells $\Phi: F \Rightarrow G$ and $\Psi: G \Rightarrow H$ for lax functors $F, G, H: I \rightarrow \mathcal{T}$ is $\Psi \circ \Phi: F \Rightarrow H$, where $(\Psi \circ \Phi) a=\Psi a \circ \Phi a: F a \Rightarrow H a$ for each arrow $a: i \rightarrow j$ in $I$. Its component

$$
(\Psi \circ \Phi)_{a, b}: H_{a, b} \circ((\Psi \circ \Phi) a \otimes(\Psi \circ \Phi) b) \Rightarrow(\Psi(a b) \circ \Phi(a b)) \circ F_{a, b},
$$

attached at a pair of composable arrows $i \stackrel{b}{\longrightarrow} j \stackrel{a}{\longrightarrow} k$ of the category $I$, is given by pasting the diagram

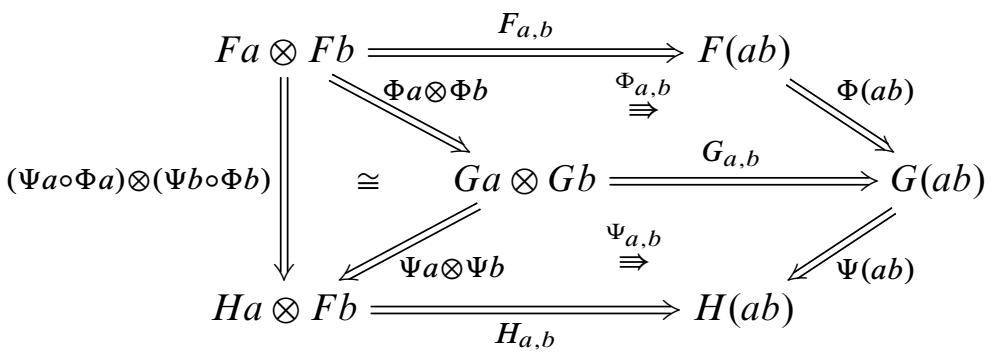

in the bicategory $\mathcal{T}(F i, F k)$, and its component $(\Psi \circ \Phi)_{i}: H_{i} \Rightarrow(\Psi \circ \Phi) 1_{i} \circ F_{i}$, attached at any object $i$ of $I$, is given by pasting the diagram

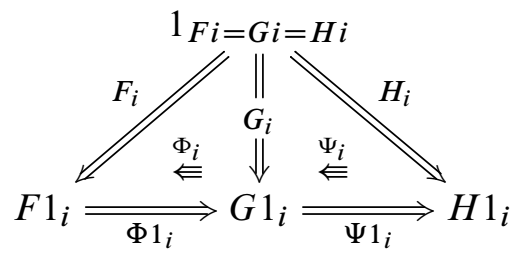

in $\mathcal{T}(F i, F i)$. The horizontal composition of 2 -cells $M: \Phi \Rightarrow \Psi: F \Rightarrow G$ and $N: \Gamma \Rightarrow \Theta: G \Rightarrow H$ in $\operatorname{Lax}(I, \mathcal{T})$ is $N \circ M: \Gamma \circ \Phi \Rightarrow \Theta \circ \Psi$, which, at each $a: i \rightarrow j$ in $I$, is given by the formula $(N \circ M) a=N a \circ M a$. 
Identities in $\operatorname{Lax}(I, \mathcal{T})$ The identity 1 -cell of a lax functor $F: I \rightarrow \mathcal{T}$ is $1_{F}: F \Rightarrow F$, where $\left(1_{F}\right) a=1_{F a}$ is the identity of $F a$ in the bicategory $\mathcal{T}(F i, F i)$ for each $a: i \rightarrow j$ in $I$. Its structure 3-cell $\left(1_{F}\right)_{a, b}: F_{a, b} \circ\left(1_{F a} \otimes 1_{F a}\right) \Rightarrow 1_{F(a b)} \otimes F_{a, b}$, attached at each pair of composable arrows $i \stackrel{b}{\longrightarrow} j \stackrel{a}{\longrightarrow} k$, is the canonical one obtained from the identity constraints of the bicategory $\mathcal{T}(F k, F i)$ by pasting the diagram

$$
\begin{aligned}
& F a \otimes F b \stackrel{F_{a, b}}{\Longrightarrow} F(a b) \\
& 1 \otimes 1 \Downarrow \cong)_{1} \cong \underset{\|}{\|} \\
& F a \otimes F b \underset{\overline{F_{a, b}}}{\longrightarrow} F(a b),
\end{aligned}
$$

and its component, attached at any object $i$ of $I$, is obtained from the left unit constraints of the bicategory $\mathcal{T}(F i, F i)$ at $F_{i}: 1_{F_{i}} \Rightarrow F 1_{i}$, that is, $\left(1_{F}\right)_{i}=\boldsymbol{l}^{-1}: F_{i} \cong 1_{F 1_{i}} \circ F_{i}$. The identity $2-$ cell $1_{\Phi}$ of a $1-$ cell $\Phi: F \Rightarrow G$ is defined at any arrow $a: i \rightarrow j$ of $I$ by the simple formula $\left(1_{\Phi}\right) a=1_{\Phi a}: \Phi a \Rightarrow \Phi a$.

The structure constraints in $\operatorname{Lax}(I, \mathcal{T})$ For any three composable 1-cells

$$
F \stackrel{\Phi}{\Longrightarrow} G \stackrel{\Psi}{\Longrightarrow} H \stackrel{\Theta}{\Longrightarrow} K
$$

in $\operatorname{Lax}(I, T)$, the component of the structure associativity isomorphism $(\Theta \circ \Psi) \circ \Phi \cong$ $\Theta \circ(\Psi \circ \Phi)$, at any arrow $i \stackrel{a}{\longrightarrow} j$ of the category $I$, is provided by the associativity constraint $(\Theta a \circ \Psi a) \circ \Phi a \cong \Theta a \circ(\Psi a \circ \Phi a)$ of the hom bicategory $\mathcal{T}(F i, F j)$. And similarly, the components of structure left and right identity isomorphisms $1_{G} \circ \Phi \cong \Phi$ and $\Phi \circ 1_{F} \cong \Phi$, at any arrow $a: i \rightarrow j$ as above, are provided by the identity constraints $1_{G a} \circ \Phi a \cong \Phi a$, and $\Phi a \circ 1_{F a} \cong \Phi a$, of the bicategory $\mathcal{T}(F i, F j)$, respectively.

The bicategory $\operatorname{Lax}(I, \mathcal{T})$, contains three sub-bicategories that are of interest in our development: The bicategory of unitary lax functors, denoted by

$$
\operatorname{Lax}_{\mathrm{u}}(I, \mathcal{T})
$$

is the bicategory whose 1-cells are those $\Phi: F \Rightarrow G$ in $\operatorname{Lax}(I, \mathcal{T})$ that are unitary in the sense that $\Phi 1_{i}=1_{1_{F i}}$, the 3 -cells $\Phi_{1, a}, \Phi_{a, 1}$ in (3) and $\Phi_{i}$ in (4) are those given by the constraints of the tricategory, and it is full on 2-cells between such normalized $1-$ cells.

The bicategory of homomorphic lax functors (ie of trihomomorphisms), denoted by

$$
\operatorname{Lax}_{\mathrm{h}}(I, \mathcal{T})
$$


is the bicategory whose 1 -cells are those $\Phi: F \Rightarrow G$ in $\operatorname{Lax}(I, \mathcal{T})$ such that the structure 3-cells $\Phi_{a, b}$ and $\Phi_{i}$ are all invertible, and it is full on 2-cells $M: \Phi \Rightarrow \Psi$ between such $1-$ cells.

The bicategory of unitary homomorphic lax functors, denoted by $\operatorname{Lax}_{\mathrm{uh}}(I, \mathcal{T})$, is defined to be the intersection of the above two, that is,

$$
\operatorname{Lax}_{\mathrm{uh}}(I, \mathcal{T})=\operatorname{Lax}_{\mathrm{u}}(I, \mathcal{T}) \cap \mathbb{L} \operatorname{ax}_{\mathrm{h}}(I, \mathcal{T})
$$

Example 2.2 Let $\Sigma^{2} A$ be the strict tricategory defined by an abelian group $A$ as in Example 2.1 and let $I$ be any category. Then the bicategory $\operatorname{Lax}_{\mathrm{u}}\left(I, \Sigma^{2} A\right)$ is actually a 2-groupoid whose objects are normalized 3-cocycles of $I$ with coefficients in $A$. If $F, G: \mathrm{N}_{3} I \rightarrow A$ are two such 3 -cocycles, then a $1-$ cell $\Phi: F \Rightarrow G$ is a normalized 2-cochain $\Phi: \mathrm{N}_{2} I \rightarrow A$ satisfying

$$
G(a, b, c)+\Phi(b, c)+\Phi(a, b c)=F(a, b, c)+\Phi(a b, c)+\Phi(a, b),
$$

that is, $G=F+\partial \Phi$. And for any two 1-cells $\Phi, \Psi: F \Rightarrow G$ as above, a 2-cell $M: \Phi \Rightarrow \Psi$ consists of a normalized $1-$ cochain $M: \mathrm{N}_{1} I \rightarrow A$ such that $\Psi=\Phi+\partial M$, that is, such that $\Psi(a, b)+M(a)+M(b)=M(a b)+\Phi(a, b)$.

\subsection{Functorial properties of $\operatorname{Lax}(I,-)$}

For any given tricategory $\mathcal{T}$, any functor $\alpha: I \rightarrow J$ induces a strict functor

$$
\alpha^{*}: \mathbb{L a x}(J, \mathcal{T}) \rightarrow \mathbb{L} \operatorname{ax}(I, \mathcal{T})
$$

given on cells in the following way: for any $F: J \rightarrow \mathcal{T}, \alpha^{*} F: I \rightarrow \mathcal{T}$ is the lax functor acting both on objects and arrows as the composite $F \alpha$, and whose structure cells are simply given by the rules

$$
\begin{gathered}
\left(\alpha^{*} F\right)_{a, b}=F_{\alpha a, \alpha b}, \quad\left(\alpha^{*} F\right)_{i}=F_{\alpha i}, \\
\left(\alpha^{*} F\right)_{a, b, c}=F_{\alpha a, \alpha b, \alpha c}, \quad\left(\widehat{\alpha^{*} F}\right)_{a}=\widehat{F}_{\alpha a}, \quad\left(\widetilde{\alpha^{*} F}\right)_{a}=\widetilde{F}_{\alpha a} .
\end{gathered}
$$

Notice that $\alpha^{*} F$ is slightly different from the composite lax functor $F \alpha$, which will give structure 2-cells $(F \alpha)_{a, b}=1_{F(\alpha a \alpha b)} \circ F_{\alpha a, \alpha b}$.

For $\Phi: F \Rightarrow G$ a 1 -cell of $\operatorname{Lax}(J, \mathcal{T}), \alpha^{*} \Phi: \alpha^{*} F \Rightarrow \alpha^{*} G$ is the $1-$ cell of $\operatorname{Lax}(I, \mathcal{T})$ with

$$
\left(\alpha^{*} \Phi\right) a=\Phi(\alpha a), \quad\left(\alpha^{*} \Phi\right)_{a, b}=\Phi_{\alpha a, \alpha b}, \quad\left(\alpha^{*} \Phi\right)_{i}=\Phi_{\alpha i} .
$$

Similarly, for any 2-cell $M: \Phi \Rightarrow \Psi$ in $\operatorname{Lax}(J, \mathcal{T}), \alpha^{*} M: \alpha^{*} \Phi \Rightarrow \alpha^{*} \Psi$ is the 2-cell of $\operatorname{Lax}(I, \mathcal{T})$ with $\left(\alpha^{*} M\right) a=M(\alpha a)$. 
Using the definition above, the construction $I \mapsto \mathbb{L a x}(I, \mathcal{T})$ is functorial on the category $I$. For a trihomomorphism of tricategories $H=(H, \chi, \iota, \omega, \gamma, \delta): \mathcal{T} \rightarrow \mathcal{T}^{\prime}$, as defined by Gordon, Power and Street in [20, Definition 3.1], we have the following result.

Lemma 2.3 Let $I$ be any given small category.

(i) Every trihomomorphism $H: \mathcal{T} \rightarrow \mathcal{T}^{\prime}$ gives rise to a homomorphism

$$
H_{*}: \operatorname{Lax}(I, \mathcal{T}) \rightarrow \mathbb{L a x}\left(I, \mathcal{T}^{\prime}\right)
$$

that is natural on $I$, that is, for any functor $\alpha: I \rightarrow J$,

$$
H_{*} \alpha^{*}=\alpha^{*} H_{*}: \mathbb{L a x}(J, \mathcal{T}) \rightarrow \operatorname{Lax}\left(I, \mathcal{T}^{\prime}\right) .
$$

(ii) If $H: \mathcal{T} \rightarrow \mathcal{T}^{\prime}$ and $H^{\prime}: \mathcal{T}^{\prime} \rightarrow \mathcal{T}^{\prime \prime}$ are any two composable trihomomorphisms, then there is a pseudoequivalence $m: H_{*}^{\prime} H_{*} \Rightarrow\left(H^{\prime} H\right)_{*}$ such that, for any functor $\alpha: I \rightarrow J$, the equality $m \alpha^{*}=\alpha^{*} m$ holds.

(iii) For any tricategory $\mathcal{T}$, there is a pseudoequivalence $m:\left(1_{\mathcal{T}}\right)_{*} \Rightarrow 1$ such that, for any functor $\alpha: I \rightarrow J$, the equality $m \alpha^{*}=\alpha^{*} m$ holds.

Proof This is given in Section A.1.

\subsection{Lax functors from free categories}

Let us now replace the category $I$ above by a (directed) graph $\mathcal{G}$. For any tricategory $\mathcal{T}$ there is a bicategory

$$
\operatorname{Lax}(\mathcal{G}, \mathcal{T}),
$$

where a 0 -cell $f: \mathcal{G} \rightarrow \mathcal{T}$ consists of a pair of maps that assign an object $f i$ to each vertex $i \in \mathcal{G}$ and a 1-cell $f a: f i \rightarrow f j$ to each edge $a: i \rightarrow j$ in $\mathcal{G}$, respectively. A $1-$ cell $\phi: f \Rightarrow g$ may exist only if $f$ and $g$ agree on vertices, that is, $f i=g i$ for all $i \in \mathcal{G}$; and then it consists of a map that assigns to each edge $a: i \rightarrow j$ in the graph a 2-cell $\phi a: f a \Rightarrow g a$ of $\mathcal{T}$. And a 2-cell $m: \phi \Rightarrow \psi$, for $\phi, \psi: f \Rightarrow g$ two 1 -cells as above, consists of a family of 3-cells in $\mathcal{T}, m a: \phi a \equiv \psi a$, one for each arrow $a: i \rightarrow j$ in $I$. Compositions in $\operatorname{Lax}(\mathcal{G}, \mathcal{T})$ are defined in the natural way by the same rules as those stated above for the bicategory of lax functors from a category into a tricategory.

Suppose now that $I(\mathcal{G})$ is the free category generated by the graph $\mathcal{G}$. Then, restriction to the basic graph gives a strict functor

$$
R: \mathbb{L a x}(I(\mathcal{G}), \mathcal{T}) \rightarrow \operatorname{Lax}(\mathcal{G}, \mathcal{T}),
$$

and we shall state the following auxiliary statement to be used later. 
Lemma 2.4 Let $I=I(\mathcal{G})$ be the free category generated by a graph $\mathcal{G}$. Then for any tricategory $\mathcal{T}$, there is a homomorphism

$$
L: \mathbb{L a x}(\mathcal{G}, \mathcal{T}) \rightarrow \mathbb{L a x}(I, \mathcal{T})
$$

and a lax transformation $\mathrm{v}: L R \Rightarrow 1_{\mathbb{L a x}(I, \mathcal{T})}$ such that the following facts hold.

(i) $R L=1_{\mathbb{L a x}(\mathcal{G}, \mathcal{T})}, \mathrm{v} L=1_{L}, R \mathrm{v}=1_{R}$

(ii) The image of $L$ is contained into the sub-bicategory $\operatorname{Lax}_{\mathrm{uh}}(I, \mathcal{T}) \subseteq \mathbb{L a x}(I, \mathcal{T})$.

(iii) The restricted homomorphisms of $L$ and $R$ establish biadjoint biequivalences

$$
\begin{aligned}
& \operatorname{Lax}(\mathcal{G}, \mathcal{T}) \underset{R}{\stackrel{L}{\underset{\sim}{\sim}}} \operatorname{Lax}_{\mathrm{h}}(I, \mathcal{T}), \\
& \operatorname{Lax}(\mathcal{G}, \mathcal{T}) \underset{R}{\stackrel{L}{\sim}} \operatorname{Lax}_{\mathrm{uh}}(I, \mathcal{T})
\end{aligned}
$$

whose respective unit is the identity $1: 1 \Rightarrow R L$, whose counit is given by the corresponding restriction of $\mathrm{v}: L R \Rightarrow 1$, and whose triangulators are the canonical modifications $1 \cong 1 \circ 1=\mathrm{v} L \circ L 1$ and $R \mathrm{v} \circ 1 R=1 \circ 1 \cong 1$, respectively.

Proof This is given in Section A.2.

\section{The Grothendieck nerve of a tricategory}

Let us briefly recall that it was Grothendieck who first associated a simplicial set

$$
\mathrm{NC}: \Delta^{\mathrm{op}} \rightarrow \text { Set }
$$

to a small category $C$, calling it its nerve. The set of $p$-simplices

$$
\mathrm{N}_{p} C=\bigsqcup_{\left(c_{0}, \ldots, c_{p}\right)} C\left(c_{p-1}, c_{p}\right) \times C\left(c_{p-2}, c_{p-1}\right) \times \cdots \times C\left(c_{0}, c_{1}\right)
$$

consists of length- $p$ sequences of composable morphisms in $C$. A geometric realization of its nerve is the classifying space of the category, $\mathrm{B} C$. The main result here shows how the Grothendieck nerve construction for categories rises to tricategories.

When a tricategory $\mathcal{T}$ is strict, that is, a 3-category, then the nerve construction (9) actually gives a simplicial 2-category (see Example 4.11). For an arbitrary tricategory, the device is more complicated since the compositions of cells in a tricategory is in general not associative and not unitary (which is crucial for the simplicial structure in the construction of $\mathrm{N} \mathcal{T}$ as above), but it is only so up to coherent isomorphisms. This 
'defect' has the effect of forcing one to deal with the classifying space of a nerve of $\mathcal{T}$ in which the simplicial identities are replaced by coherent isomorphisms, that is, a pseudosimplicial bicategory as stated in the theorem below. Pseudosimplicial bicategories and the tricategory they form (whose 1-cells are pseudosimplicial homomorphisms, 2-cells pseudosimplicial transformations and 3-cells pseudosimplicial modifications) were studied by Carrasco, Cegarra and Garzón [12].

Theorem 3.1 Any tricategory $\mathcal{T}$ defines a normal pseudosimplicial bicategory, that is, a unitary trihomomorphism from the simplicial category $\Delta^{\mathrm{op}}$ into the tricategory of bicategories,

$$
\mathrm{N} \mathcal{T}=(\mathrm{N} \mathcal{T}, \chi, \omega): \Delta^{\mathrm{op}} \rightarrow \text { Bicat },
$$

called the nerve of the tricategory, whose bicategory of $p$-simplices for $p \geq 1$ is

$$
\mathrm{N}_{p} \mathcal{T}=\bigsqcup_{\left(t_{0}, \ldots, t_{p}\right) \in \mathrm{Ob} \mathcal{T}^{p+1}} \mathcal{T}\left(t_{p-1}, t_{p}\right) \times \mathcal{T}\left(t_{p-2}, t_{p-1}\right) \times \cdots \times \mathcal{T}\left(t_{0}, t_{1}\right),
$$

and $\mathrm{N}_{0} \mathcal{T}=\mathrm{Ob} \mathcal{T}$ as a discrete bicategory. The face and degeneracy homomorphisms are defined on 0 -cells, 1 -cells and 2-cells of $\mathrm{N}_{p} \mathcal{T}$ by the ordinary formulas

$$
\begin{aligned}
& d_{i}\left(x_{p}, \ldots, x_{1}\right)= \begin{cases}\left(x_{p}, \ldots, x_{2}\right) & \text { if } i=0, \\
\left(x_{p}, \ldots, x_{i+1} \otimes x_{i}, \ldots, x_{1}\right) & \text { if } 0<i<p, \\
\left(x_{p-1}, \ldots, x_{1}\right) & \text { if } i=p,\end{cases} \\
& s_{i}\left(x_{p}, \ldots, x_{1}\right)=\left(x_{p}, \ldots, x_{i+1}, 1, x_{i}, \ldots, x_{1}\right) .
\end{aligned}
$$

Indeed, if $a:[q] \rightarrow[p]$ is any map in the simplicial category $\Delta$, then the associated homomorphism $\mathrm{N}_{a} \mathcal{T}: \mathrm{N}_{p} \mathcal{T} \rightarrow \mathrm{N}_{q} \mathcal{T}$ is induced by the composition

$$
\mathcal{T}\left(t^{\prime}, t\right) \times \mathcal{T}\left(t^{\prime \prime}, t^{\prime}\right) \stackrel{\otimes}{\longrightarrow} \mathcal{T}\left(t^{\prime \prime}, t\right)
$$

and unit $1_{t}: 1 \rightarrow \mathcal{T}(t, t)$ homomorphisms. The structure pseudoequivalences

$$
\mathrm{N}_{p} \mathcal{T} \underset{\mathrm{N}_{a b} \mathcal{T}}{\stackrel{\mathrm{N}_{b} \mathcal{T} \mathrm{N}_{a} \mathcal{T}}{\Downarrow_{a, b}^{\chi_{a, b}}}} \mathrm{~N}_{n} \mathcal{T},
$$

for each pair of composable maps $[n] \stackrel{b}{\longrightarrow}[q] \stackrel{a}{\longrightarrow}[p]$ in $\Delta$, and the invertible modifications

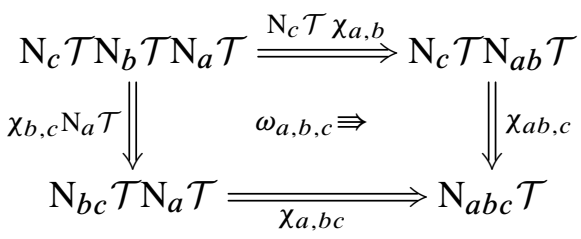


respectively, associated to triplets of composable arrows $[m] \stackrel{c}{\rightarrow}[n] \stackrel{b}{\longrightarrow}[q] \stackrel{a}{\longrightarrow}[p]$ canonically arise all from the structure pseudoequivalences and modifications data of the tricategory.

We prove Theorem 3.1 simultaneously with Proposition 3.2 below, which states the basic properties concerning the behavior of the Grothendieck nerve construction $\mathcal{T} \mapsto \mathrm{N} \mathcal{T}$ with respect to trihomomorphisms of tricategories.

Proposition 3.2 (i) Any trihomomorphism between tricategories $H: \mathcal{T} \rightarrow \mathcal{T}^{\prime}$ induces a normal pseudosimplicial homomorphism

$$
\mathrm{N} H=(\mathrm{N} H, \theta, \Pi): \mathrm{N} \mathcal{T} \rightarrow \mathrm{N} \mathcal{T}^{\prime}
$$

which, at any integer $p \geq 0$, is the evident homomorphism $\mathrm{N}_{p} H: \mathrm{N}_{p} \mathcal{T} \rightarrow \mathrm{N}_{p} \mathcal{T}^{\prime}$ defined on any cell $\left(x_{p}, \ldots, x_{1}\right)$ of $\mathrm{N}_{p} \mathcal{T}$ by

$$
\mathrm{N}_{p} H\left(x_{p}, \ldots, x_{1}\right)=\left(H x_{p}, \ldots, H x_{1}\right) .
$$

The structure pseudoequivalence

$$
\begin{gathered}
\mathrm{N}_{p} \mathcal{T} \stackrel{\mathrm{N}_{p} H}{\longrightarrow} \mathrm{N}_{p} \mathcal{T}^{\prime} \\
\mathrm{N}_{a} \mathcal{T} \downarrow \quad \theta_{a} \Rightarrow \quad \begin{array}{l}
\downarrow \mathrm{N}_{a} \mathcal{T}^{\prime} \\
\mathrm{N}_{q} \mathcal{T} \underset{\mathrm{N}_{q} H}{\longrightarrow} \mathrm{N}_{q} \mathcal{T}^{\prime}
\end{array}
\end{gathered}
$$

for each map $a:[q] \rightarrow[p]$ in $\Delta$, and the invertible modifications

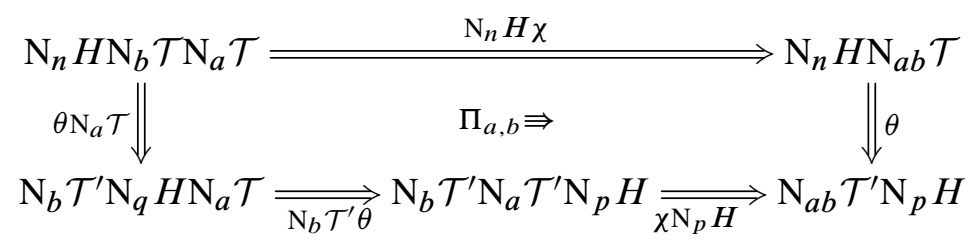

respectively, associated to pairs of composable arrows $[n] \stackrel{b}{\longrightarrow}[q] \stackrel{a}{\longrightarrow}[p]$, canonically arise all from the structure pseudoequivalences and modifications data of the trihomomorphism and the involved tricategories.

(ii) For any pair of composable trihomomorphisms $H: \mathcal{T} \rightarrow \mathcal{T}^{\prime}$ and $H^{\prime}: \mathcal{T}^{\prime} \rightarrow \mathcal{T}^{\prime \prime}$, there is a pseudosimplicial pseudoequivalence

$$
\mathrm{N} H^{\prime} \mathrm{N} H \Rightarrow \mathrm{N}\left(H^{\prime} H\right) \text {. }
$$

(iii) For any tricategory $\mathcal{T}$, there is a pseudosimplicial pseudoequivalence

$$
\mathrm{N} 1_{\mathcal{T}} \Rightarrow 1_{\mathrm{N} \mathcal{T}}
$$


Proof of Theorem 3.1 and Proposition 3.2 Let us note that, for any $p \geq 0$, the category $[p]$ is free on the graph $\mathcal{G}_{p}=(0 \rightarrow 1 \rightarrow \cdots \rightarrow p)$, and that $\mathrm{N}_{p} \mathcal{T}=\mathbb{L a x}\left(\mathcal{G}_{p}, \mathcal{T}\right)$. Hence the existence of a biadjoint biequivalence

$$
L_{p} \dashv R_{p}: \mathrm{N}_{p} \mathcal{T} \rightleftarrows \mathbb{L} \operatorname{ax}_{\mathrm{h}}([p], \mathcal{T})
$$

follows from Lemma 2.4, where $R_{p}$ is the strict functor defined by restricting to the basic graph $\mathcal{G}_{p}$ of the category $[p]$, such that $R_{p} L_{p}=1$, whose unity is the identity, and whose counit $\mathrm{v}_{p}: L_{p} R_{p} \Rightarrow 1$ is a pseudoequivalence satisfying the equalities $\mathrm{v}_{p} L_{p}=1$ and $R_{p} \mathrm{v}_{p}=1$. Then if $a:[q] \rightarrow[p]$ is any map in the simplicial category, the associated homomorphism $\mathrm{N}_{a} \mathcal{T}: \mathrm{N}_{p} \mathcal{T} \rightarrow \mathrm{N}_{q} \mathcal{T}$ is defined to be the composite

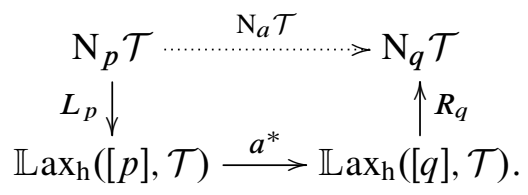

Observe that thus defined, the homomorphism $\mathrm{N}_{a} \mathcal{T}$ maps the component bicategory of $\mathrm{N}_{p} \mathcal{T}$ at $\left(t_{p}, \ldots, t_{0}\right)$ into the component at $\left(t_{a(q)}, \ldots, t_{a(0)}\right)$ of $\mathrm{N}_{q} \mathcal{T}$, and it acts on 0 -cells, 1 -cells and 2 -cells of $\mathrm{N}_{p} \mathcal{T}$ by the formula $\mathrm{N}_{a} \mathcal{T}\left(x_{p}, \ldots, x_{1}\right)=\left(y_{q}, \ldots, y_{1}\right)$, where for $0 \leq k<q$ (see (1) for the definition of $\otimes^{\text {or }}$ )

$$
y_{k+1}= \begin{cases}\otimes^{\mathrm{or}}\left(x_{a(k+1)}, \ldots, x_{a(k)+1}\right) & \text { if } a(k)<a(k+1), \\ 1 & \text { if } a(k)=a(k+1) .\end{cases}
$$

Thus we get, in particular, the formulas for the face and degeneracy homomorphisms.

The pseudonatural equivalence (11) is

$$
\mathrm{N}_{b} \mathcal{T} \mathrm{N}_{a} \mathcal{T}=R_{n} b^{*} L_{q} R_{q} a^{*} L_{p} \stackrel{\chi_{a, b}=R_{n} b^{*} \mathrm{v}_{q} a^{*} L_{p}}{\longrightarrow} R_{n} b^{*} a^{*} L_{p}=R_{n}(a b)^{*} L_{p}=\mathrm{N}_{a b} \mathcal{T}
$$

and the invertible modification (12) is

$$
\omega_{a, b, c}=R_{m} c^{*} \omega_{b}^{\prime} a^{*} L_{p},
$$

where $\omega_{b}^{\prime}$ is the canonical modification below:

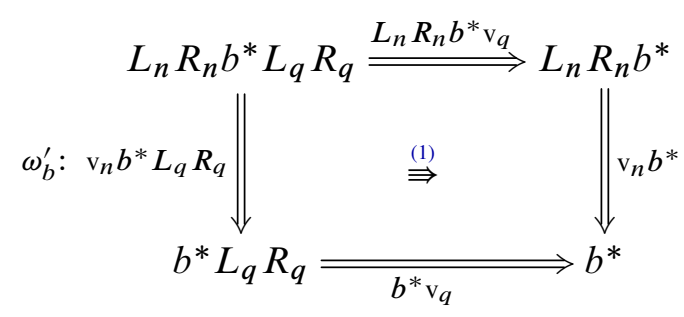


Thus defined, $\mathrm{N} \mathcal{T}$ is actually a normal pseudosimplicial bicategory. Both coherence conditions for $\mathrm{N} \mathcal{T}$, that is, [12, Conditions (CC1), (CC2)], follow from the equalities $R_{p} L_{p}=1, \mathrm{v}_{p} L_{p}=1$ and $R_{p} \mathrm{v}_{p}=1$, and coherence in the tricategory Bicat.

This proves Theorem 3.1.

When it comes to Proposition 3.2, let us note that the homomorphisms $\mathrm{N}_{p} H: \mathrm{N}_{p} \mathcal{T} \rightarrow$ $\mathrm{N}_{p} \mathcal{T}^{\prime}, p \geq 0$, make commutative the diagrams

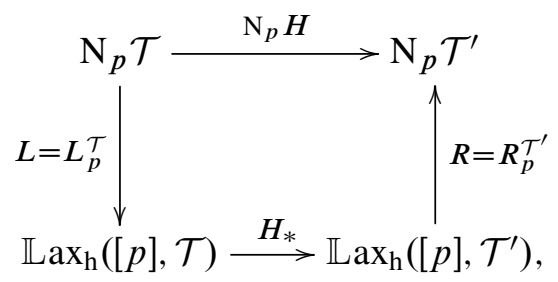

where $H_{*}$ is the induced homomorphism by the trihomomorphism $H: \mathcal{T} \rightarrow \mathcal{T}^{\prime}$, as stated in Lemma 2.3(i). Then the pseudoequivalence in (13), $\theta_{a}$, is provided by the pseudoequivalences $\mathrm{v}: L R \Rightarrow 1$ and their adjoint quasi-inverses $\mathrm{v}^{\bullet}: 1 \Rightarrow L R$ (which we can choose such that $R \mathrm{v}^{\bullet}=1$ and $\left.\mathrm{v}^{\bullet} L=1\right)$, that is, $\theta_{a}=R a^{*} \mathrm{v}^{\bullet} H_{*} L \circ R H_{*} L R a^{*} L$,

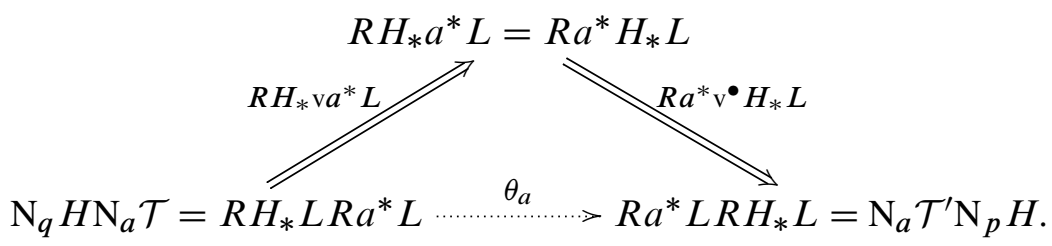

And, for $[n] \stackrel{b}{\longrightarrow}[q] \stackrel{a}{\longrightarrow}[p]$, any two composable arrows of $\Delta$, the structure invertible modification (14), $\Pi_{a, b}$, is the modification obtained by pasting the diagram

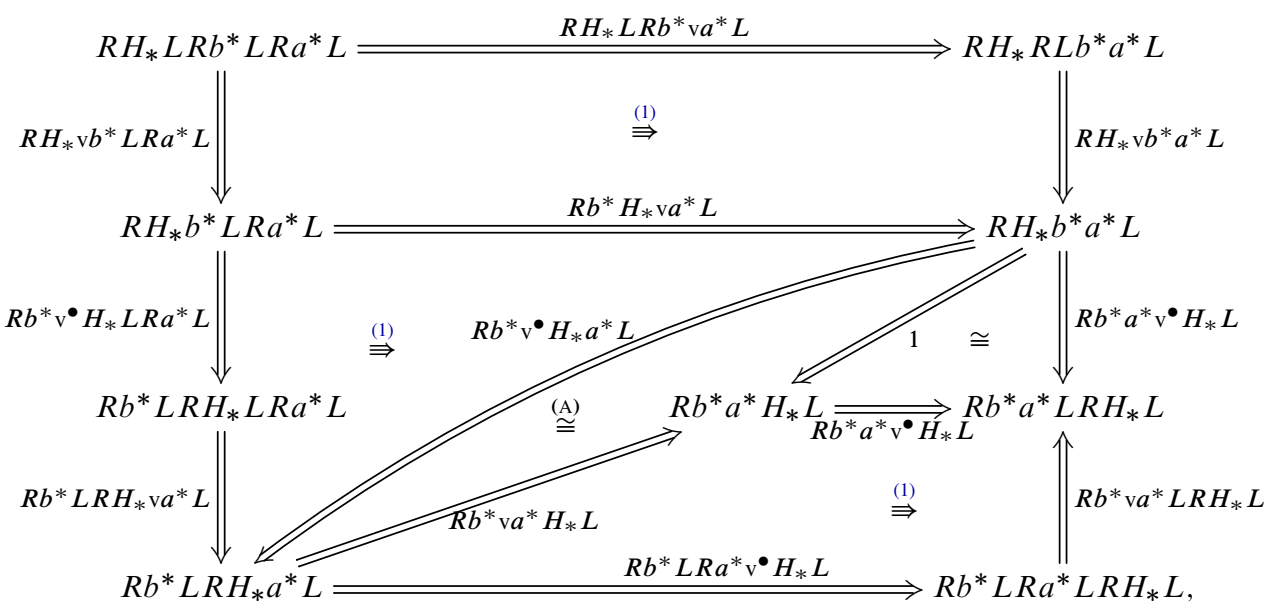


where the isomorphism labeled (A) is given by the adjunction invertible modification $\mathrm{v} \circ \mathrm{v}^{\bullet} \cong 1$. The coherence conditions for $\mathrm{N} H: \mathrm{N} \mathcal{T} \rightarrow \mathrm{N} \mathcal{T}^{\prime}$, that is [12, Conditions (CC3), (CC4)], are easily verified again from coherence in Bicat.

Suppose now that $\mathcal{T} \stackrel{H}{\longrightarrow} \mathcal{T}^{\prime} \stackrel{H^{\prime}}{\longrightarrow} \mathcal{T}^{\prime \prime}$ are two composable trihomomorphisms. Then the pseudosimplicial pseudoequivalence from (15), $\alpha: \mathrm{N} H^{\prime} \mathrm{N} H \Rightarrow \mathrm{N}\left(H^{\prime} H\right)$, is at any integer $p \geq 0$ given by $\alpha_{p}=R m L \circ R H_{*}^{\prime} \mathrm{v} H_{*} L$,

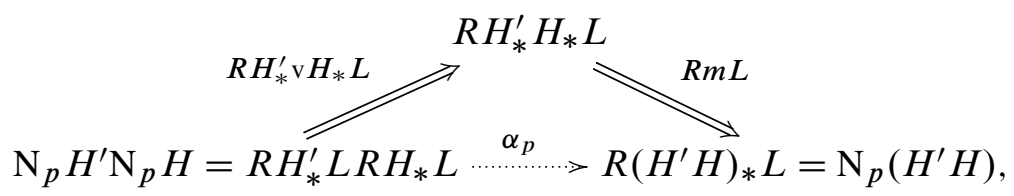

where the pseudoequivalence $m: H_{*}^{\prime} H_{*} \Rightarrow\left(H^{\prime} H\right)_{*}: \operatorname{Lax}_{\mathrm{h}}([p], \mathcal{T}) \rightarrow \mathbb{L} \operatorname{Lax}_{\mathrm{h}}\left([p], \mathcal{T}^{\prime \prime}\right)$ is that given in Lemma 2.3(ii). The naturality component of $\alpha$ at any map $a:[q] \rightarrow[p]$,

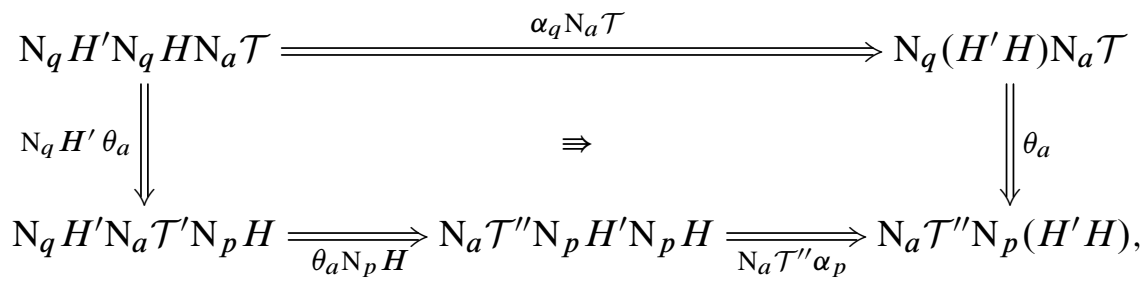

is provided by the invertible modification obtained by pasting in

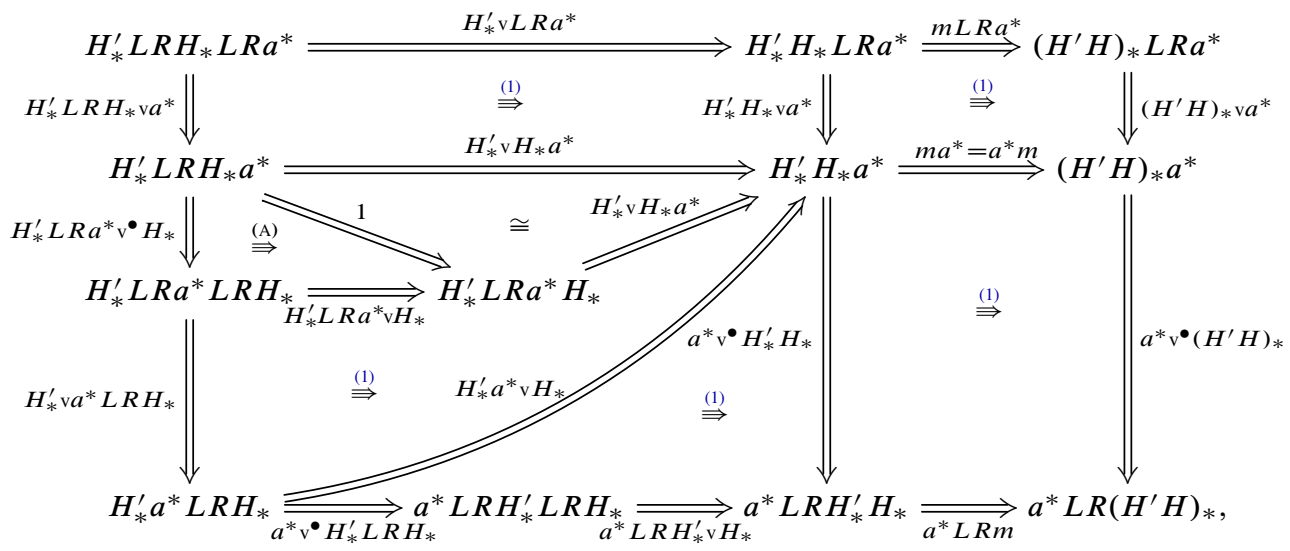

where the isomorphism (A) is given by the adjunction invertible modification $\mathrm{v}^{\bullet} \mathrm{v}^{\bullet} \cong 1$.

Finally, the pseudosimplicial pseudoequivalence (16), $\beta: \mathrm{N} 1_{\mathcal{T}} \Rightarrow 1_{\mathrm{N} \mathcal{T}}$, is defined by the family of pseudoequivalences

$$
\mathrm{N}_{p} 1_{\mathcal{T}}=R\left(1_{\mathcal{T}}\right)_{*} L \stackrel{\beta_{p}=R m L}{\longrightarrow} R L=1_{\mathrm{N}_{p} \mathcal{T}},
$$


where $m:\left(1_{\mathcal{T}}\right)_{*} \Rightarrow 1: \mathbb{L a x}_{\mathrm{h}}([p], \mathcal{T}) \rightarrow \mathbb{L a x}_{\mathrm{h}}([p], \mathcal{T})$ is the pseudoequivalence in Lemma 2.3(iii). The naturality invertible modification attached at any map $a:[q] \rightarrow[p]$,

$$
\begin{array}{ccc}
\mathrm{N}_{p} 1_{\mathcal{T}} \mathrm{N}_{a} \mathcal{T} & \stackrel{\beta_{p} \mathrm{~N}_{a} \mathcal{T}}{\Longrightarrow} \mathrm{N}_{a} \mathcal{T} \\
\theta_{a} \Downarrow & \Rightarrow \quad \\
\mathrm{N}_{a} \mathcal{T} \mathrm{N}_{q} 1_{\mathcal{T}} & \underset{\mathrm{N}_{a} \mathcal{T} \beta_{q}}{\Longrightarrow} & \mathrm{N}_{a} \mathcal{T},
\end{array}
$$

is the one obtained by pasting the diagram

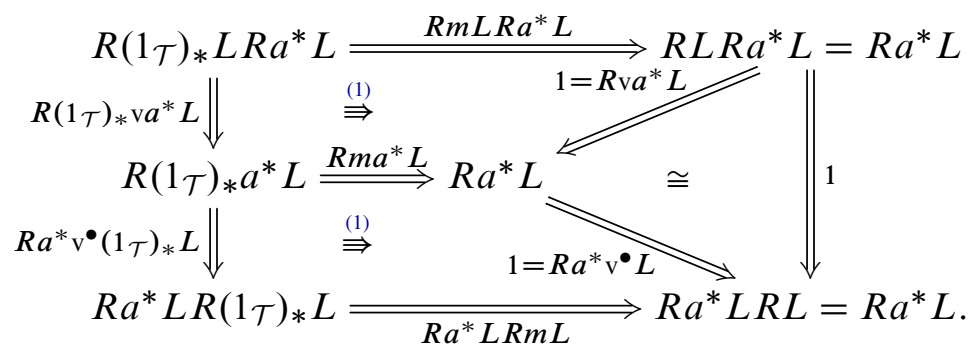

Conditions (CC5) and (CC6) in [12], for both $\alpha$ and $\beta$, are plainly verified.

\section{The classifying space of a tricategory}

\subsection{Preliminaries on classifying spaces of bicategories}

When a bicategory $\mathcal{B}$ is regarded as a tricategory whose 3 -cells are all identities, the nerve construction on it actually produces a normal pseudosimplicial category $\mathrm{NB}=(\mathrm{N} \mathcal{B}, \chi): \Delta^{\mathrm{op}} \rightarrow$ Cat, which was called the pseudosimplicial nerve of the bicategory by Carrasco, Cegarra and Garzón [11, Section 3]. The classifying space of the bicategory, denoted here by $\mathrm{B}_{2} \mathcal{B}$, is then defined to be the ordinary classifying space of the category obtained by the Grothendieck construction [21] on the nerve of the bicategory, that is,

$$
\mathrm{B}_{2} \mathcal{B}=\mathrm{B} \int_{\Delta} \mathrm{N} \mathcal{B}
$$

The following facts are proved in [11].

Fact 4.1 Each homomorphism between bicategories $F: \mathcal{B} \rightarrow \mathcal{C}$ induces a continuous (cellular) map $\mathrm{B}_{2} F: \mathrm{B}_{2} \mathcal{B} \rightarrow \mathrm{B}_{2} \mathcal{C}$. Thus, the classifying space construction, $\mathcal{B} \mapsto \mathrm{B}_{2} \mathcal{B}$, defines a functor from the category Hom of bicategories to $\mathrm{CW}$-complexes. 
Fact 4.2 If $F, F^{\prime}: \mathcal{B} \rightarrow \mathcal{C}$ are two homomorphisms between bicategories, then any lax (or oplax) transformation $F \Rightarrow F^{\prime}$ canonically defines a homotopy between the induced maps on classifying spaces, $\mathrm{B}_{2} F \simeq \mathrm{B}_{2} F^{\prime}: \mathrm{B}_{2} \mathcal{B} \rightarrow \mathrm{B}_{2} \mathcal{C}$.

Fact 4.3 If a homomorphism of bicategories has a left or right biadjoint, the map induced on classifying spaces is a homotopy equivalence. In particular, any biequivalence of bicategories induces a homotopy equivalence on classifying spaces.

We also recall that the classifying space of any pseudosimplicial bicategory $\mathcal{F}: \Delta^{\mathrm{op}} \rightarrow$ Bicat is defined by Carrasco, Cegarra and Garzón in [12, Definition 5.4] to be the classifying space of its bicategory of simplices $\int_{\Delta} \mathcal{F}$, also called the Grothendieck construction on $\mathcal{F}[12$, Section 3.1]. That is, the bicategory whose objects are the pairs $(x, p)$, where $p \geq 0$ is an integer and $x$ is an object of the bicategory $\mathcal{F}_{p}$, and whose hom categories are

$$
\int_{\Delta} \mathcal{F}((y, q),(x, p))=\bigsqcup_{[q] \stackrel{a}{\longrightarrow}[p]} \mathcal{F}_{q}\left(y, a^{*} x\right),
$$

where the disjoint union is over all arrows $a:[q] \rightarrow[p]$ in the simplicial category $\Delta$; compositions, identities and structure constraints are defined in the natural way. We refer the reader to [12, Section 3] for details about the bicategorical Grothendieck construction trihomomorphism

$$
\int_{\Delta}-: \text { Bicat }^{\Delta^{\mathrm{op}}} \rightarrow \text { Bicat }
$$

from the tricategory of pseudosimplicial bicategories to the tricategory of bicategories. The following facts are proved in [12].

Fact 4.4 (i) If $\mathcal{F}, \mathcal{G}: \Delta^{\mathrm{op}} \rightarrow$ Bicat are pseudosimplicial bicategories, then each pseudosimplicial homomorphism $F: \mathcal{F} \rightarrow \mathcal{G}$ induces a continuous map

$$
\mathrm{B}_{2} \int_{\Delta} F: \mathrm{B}_{2} \int_{\Delta} \mathcal{F} \rightarrow \mathrm{B}_{2} \int_{\Delta} \mathcal{G} .
$$

(ii) For any pseudosimplicial bicategory $\mathcal{F}: \Delta^{\mathrm{op}} \rightarrow$ Bicat, there is a homotopy

$$
\mathrm{B}_{2} \int_{\Delta} 1_{\mathcal{F}} \simeq 1_{\mathrm{B}_{2} \int_{\Delta} \mathcal{F}}: \mathrm{B}_{2} \int_{\Delta} \mathcal{F} \rightarrow \mathrm{B}_{2} \int_{\Delta} \mathcal{F} .
$$

(iii) For any pair of composable pseudosimplicial homomorphism $F: \mathcal{F} \rightarrow \mathcal{G}$, $G: \mathcal{G} \rightarrow \mathcal{H}$, there is a homotopy

$$
\mathrm{B}_{2} \int_{\Delta} G \mathrm{~B}_{2} \int_{\Delta} F \simeq \mathrm{B}_{2} \int_{\Delta}(G F): \mathrm{B}_{2} \int_{\Delta} \mathcal{F} \rightarrow \mathrm{B}_{2} \int_{\Delta} \mathcal{H} .
$$

Fact 4.5 Any pseudosimplicial transformation $F \Rightarrow G: \mathcal{F} \rightarrow \mathcal{G}$ induces a homotopy

$$
\mathrm{B}_{2} \int_{\Delta} F \simeq \mathrm{B}_{2} \int_{\Delta} G: \mathrm{B}_{2} \int_{\Delta} \mathcal{F} \rightarrow \mathrm{B}_{2} \int_{\Delta} \mathcal{G} .
$$


Fact 4.6 If $F: \mathcal{F} \rightarrow \mathcal{G}$ is pseudosimplicial homomorphism, between pseudosimplicial bicategories $\mathcal{F}, \mathcal{G}: \Delta^{\mathrm{op}} \rightarrow$ Bicat, such that the induced map $\mathrm{B}_{2} F_{p}: \mathcal{B F}_{p} \rightarrow \mathrm{B}_{2} \mathcal{G}_{p}$ is a homotopy equivalence for all $p \geq 0$, then the induced map $\mathrm{B}_{2} \int_{\Delta} F: \mathrm{B}_{2} \int_{\Delta} \mathcal{F} \rightarrow \mathrm{B} \int_{\Delta} \mathcal{G}$ is a homotopy equivalence.

Fact 4.7 If $\mathcal{F}: \Delta^{\mathrm{op}} \rightarrow$ Hom $\subset$ Bicat is a simplicial bicategory, then there is a natural homotopy equivalence

$$
\mathrm{B}_{2} \int_{\Delta} \mathcal{F} \simeq\left|\mathrm{B}_{2} \mathcal{F}\right|=\left|[p] \mapsto \mathrm{B}_{2} \mathcal{F}_{p}\right|
$$

where the latter is the geometric realization of the simplicial space $\mathrm{B}_{2} \mathcal{F}: \Delta^{\mathrm{op}} \rightarrow$ Top, obtained by composing $\mathcal{F}$ with the classifying space functor $\mathrm{B}_{2}$ : Hom $\rightarrow$ Top.

\subsection{The classifying space construction for tricategories}

We are now ready to make the following definition, which recovers the more traditional way in the literature through which a classifying space is assigned to certain specific kinds of tricategories, such as 3-categories, bicategories, monoidal categories or braided monoidal categories (see Examples 4.11, 4.15 and 4.18 below).

Definition 4.8 The classifying space $\mathrm{B}_{3} \mathcal{T}$, of a tricategory $\mathcal{T}$, is the classifying space of its bicategorical pseudosimplicial Grothendieck nerve, $\mathrm{N} \mathcal{T}: \Delta^{\mathrm{op}} \rightarrow$ Bicat, that is,

$$
\mathrm{B}_{3} \mathcal{T}=\mathrm{B}_{2} \int_{\Delta} \mathrm{N} \mathcal{T}
$$

Let us remark that the classifying space of a tricategory $\mathcal{T}$ is then realized as the classifying space of a category canonically associated to it, namely, as

$$
\mathrm{B}_{3} \mathcal{T}=\mathrm{B} \int_{\Delta} \mathrm{N}\left(\int_{\Delta} \mathrm{N} \mathcal{T}\right)=\left|\mathrm{N}\left(\int_{\Delta} \mathrm{N}\left(\int_{\Delta} \mathrm{N} \mathcal{T}\right)\right)\right|
$$

Example 4.9 (Classifying spaces of categories and bicategories) When a bicategory $\mathcal{B}$ is viewed as a tricategory whose 3 -cells are all identities, then its classifying space is homotopy equivalent to the classifying space of the bicategory $\mathrm{B}_{2} \mathcal{B}$ as defined Carrasco, Cegarra and Garzón in [11, Definition 3.1], that is,

$$
\mathrm{B}_{3} \mathcal{B} \simeq \mathrm{B}_{2} \mathcal{B}
$$

To see that, let us recall that for any simplicial set $X: \Delta^{\mathrm{op}} \rightarrow$ Simpl.Set, there is a natural homotopy equivalence $\mathrm{B} \int_{\Delta} X \simeq|X|$ between the classifying space of its category of simplices and its geometric realization (see Illusie [27, Theorem 3.3]). 
Since $\mathrm{N}\left(\int_{\Delta} \mathrm{N} \mathcal{B}\right)$ is actually a simplicial set for any bicategory $\mathcal{B}$, we have a homotopy equivalence

$$
\mathrm{B}_{3} \mathcal{B}=\mathrm{B} \int_{\Delta} \mathrm{N}\left(\int_{\Delta} \mathrm{N} \mathcal{B}\right) \simeq\left|\mathrm{N}\left(\int_{\Delta} \mathrm{N} \mathcal{B}\right)\right|=\mathrm{B} \int_{\Delta} \mathrm{N} \mathcal{B}=\mathrm{B}_{2} \mathcal{B}
$$

Similarly, for any category $C$ regarded as a tricategory whose 2- and 3-cells are all identities, we have homotopy equivalences

$$
\mathrm{B}_{3} C \simeq \mathrm{B}_{2} C=\mathrm{B} \int_{\Delta} \mathrm{NC} \simeq|\mathrm{N} C|=\mathrm{B} C
$$

since $\mathrm{NC}$ is a simplicial set in this case.

The next two theorems deal with the basic properties of the homotopy behavior of the classifying space construction $\mathcal{T} \mapsto \mathrm{B}_{3} T$ with respect to trihomomorphisms of tricategories.

Proposition 4.10 (i) Any trihomomorphism between tricategories $H: \mathcal{T} \rightarrow \mathcal{T}^{\prime}$ induces a continuous (cellular) map $\mathrm{B}_{3} H: \mathrm{B}_{3} \mathcal{T} \rightarrow \mathrm{B}_{3} \mathcal{T}^{\prime}$.

(ii) For any pair of composable trihomomorphisms $H: \mathcal{T} \rightarrow \mathcal{T}^{\prime}$ and $H^{\prime}: \mathcal{T}^{\prime} \rightarrow \mathcal{T}^{\prime \prime}$, there is a homotopy

$$
\mathrm{B}_{3} H^{\prime} \mathrm{B}_{3} H \simeq \mathrm{B}_{3}\left(H^{\prime} H\right): \mathrm{B}_{3} \mathcal{T} \rightarrow \mathrm{B}_{3} \mathcal{T}^{\prime \prime} .
$$

(iii) For any tricategory $\mathcal{T}$, there is a homotopy $\mathrm{B}_{3} 1_{\mathcal{T}} \simeq 1_{\mathrm{B}_{3} \mathcal{T}}$ : $\mathrm{B}_{3} \mathcal{T} \rightarrow \mathrm{B}_{3} \mathcal{T}$.

Proof (i) By Proposition 3.2(i), any trihomomorphism $H: \mathcal{T} \rightarrow \mathcal{T}^{\prime}$ gives rise to a pseudosimplicial homomorphism on the corresponding Grothendieck nerves $\mathrm{N} H: \mathrm{N} \mathcal{T} \rightarrow \mathrm{N} \mathcal{T}^{\prime}$, which, by Fact 4.4(i), produces the claimed continuous map $\mathrm{B}_{3} H=\mathrm{B}_{2} \int_{\Delta} \mathrm{N} H: \mathrm{B}_{3} \mathcal{T} \rightarrow \mathrm{B}_{3} \mathcal{T}^{\prime}$.

(ii) Suppose that $\mathcal{T} \stackrel{H}{\longrightarrow} \mathcal{T}^{\prime} \stackrel{H^{\prime}}{\longrightarrow} \mathcal{T}^{\prime \prime}$ are trihomomorphisms. By Proposition 3.2(ii), there is a pseudosimplicial pseudoequivalence $\mathrm{N} H^{\prime} \mathrm{N} H \Rightarrow \mathrm{N}\left(H^{\prime} H\right)$, which, by Fact 4.5, induces a homotopy

$$
\mathrm{B}_{2} \int_{\Delta}\left(\mathrm{N} H^{\prime} \mathrm{N} H\right) \simeq \mathrm{B}_{2} \int_{\Delta} \mathrm{N}\left(H^{\prime} H\right)=\mathrm{B}_{3}\left(H^{\prime} H\right) .
$$

Then, the result follows since, by Fact 4.4(iii), there is a homotopy

$$
\mathrm{B}_{2} \int_{\Delta}\left(\mathrm{N} H^{\prime} \mathrm{N} H\right) \simeq \mathrm{B}_{2} \int_{\Delta} \mathrm{N}^{\prime} \mathrm{B}_{2} \int_{\Delta} \mathrm{N} H=\mathrm{B}_{3} H^{\prime} \mathrm{B}_{3} H .
$$

(iii) By Proposition 3.2(iii), we have that there is a pseudosimplicial pseudoequivalence $\mathrm{N} 1_{\mathcal{T}} \Rightarrow 1_{\mathrm{N} \mathcal{T}}$, which, by Fact 4.5 , induces a homotopy $\mathrm{B}_{3} 1_{\mathcal{T}}=\mathrm{B}_{2} \int_{\Delta} \mathrm{N} 1_{\mathcal{T}} \simeq \mathrm{B}_{2} \int_{\Delta} 1_{\mathrm{N} \mathcal{T}}$. Since, by Fact 4.4(ii), there is a homotopy $B_{2} \int_{\Delta} 1_{N} \mathcal{T} \simeq B_{2} 1_{S_{\Delta}} \mathcal{T}=1_{B} \mathcal{T}$, the result follows. 
Example 4.11 (Classifying spaces of 3-categories) In [38], Segal observed that if $\mathbb{C}$ is a topological category, then its Grothendieck nerve (9) is in a natural way a simplicial space, that is, $\mathrm{N} \mathbb{C}: \Delta^{\mathrm{op}} \rightarrow$ Top. Then he defined the classifying space of a topological category $\mathbb{C}$ to be $|N \mathbb{C}|$, the realization of this simplicial space. This notion given by Segal provides, for instance, the usual definition of classifying spaces of strict bicategories, or 2-categories, and strict tricategories, or 3-categories. Thus, the classifying space of a 2 -category $\mathcal{B}$ is, by definition, the classifying space of the topological category whose space of objects is the discrete space of objects of $\mathcal{B}$, and whose hom spaces are obtained by replacing the hom categories $\mathcal{B}\left(b, b^{\prime}\right)$ by their classifying spaces. In other words, the classifying space of the 2-category is the geometric realization of the simplicial space obtained by composing NB: $\Delta^{\text {op }} \rightarrow$ Cat with the classifying space functor $\mathcal{B}$ : Cat $\rightarrow$ Top, that is, the space

$$
\left|[p] \mapsto \mathcal{B} \mathrm{N}_{p} \mathcal{B}\right|=|[p] \mapsto| \mathrm{NN}_{p} \mathcal{B}||=|[p] \mapsto|[q] \mapsto \mathrm{N}_{q} \mathrm{~N}_{p} \mathcal{B}|| \cong|\operatorname{diag} \mathrm{NN} \mathcal{B}|,
$$

where $\operatorname{diag} \mathrm{NN} \mathcal{B}$ is the diagonal simplicial set of the bisimplicial set $\mathrm{NNB}$ double nerve of $\mathcal{B},([p],[q]) \mapsto \mathrm{N}_{q} \mathrm{~N}_{p} \mathcal{B}$, and the last homeomorphism above is a consequence of Quillen's Lemma [37, page 86]. Similarly, since a 3 -category $\mathcal{T}$ is just a category enriched in the category of 2 -categories and 2-functors, that is, a category $\mathcal{T}$ endowed with 2-categorical hom sets $\mathcal{T}\left(t^{\prime}, t\right)$ in such a way that the compositions $\mathcal{T}\left(t^{\prime}, t\right) \times \mathcal{T}\left(t^{\prime \prime}, t^{\prime}\right) \rightarrow \mathcal{T}\left(t^{\prime \prime}, t\right)$ are 2-functors, by replacing the hom 2-categories $\mathcal{T}\left(t^{\prime}, t\right)$ by their classifying spaces as above, we obtain a topological category whose classifying space is the classifying space of the 3-category $\mathcal{T}$. That is, the space

$$
|[p] \mapsto| \operatorname{diag} \mathrm{NNN}_{p} \mathcal{T}||=|[p] \mapsto|[q] \mapsto \mathrm{N}_{q} \mathrm{~N}_{q} \mathrm{~N}_{p} \mathcal{T}|\cong| \operatorname{diag} \mathrm{NNN} \mathcal{T} \mid .
$$

For $\mathcal{B}$ any 2-category, Thomason's homotopy colimit theorem [47, Theorem 1.2] and a result by Bousfield and Kan [6, XII, 4.3] give the existence of natural homotopy equivalences

$$
\mathrm{B}_{2} \mathcal{B}=\mathrm{B} \int_{\Delta} \mathrm{N} \mathcal{B} \simeq\left|\operatorname{hocolim}_{\Delta} \mathrm{NN} \mathcal{B}\right| \simeq|\operatorname{diag} \mathrm{NN} \mathcal{B}|
$$

For $\mathcal{T}$ any 3-category, by Fact 4.7, we have that there is a homotopy equivalence $\mathrm{B}_{3} \mathcal{T}=\mathrm{B}_{2} \int_{\Delta} \mathrm{N} \mathcal{T} \simeq\left|[p] \mapsto \mathrm{B}_{2} \mathrm{~N}_{p} \mathcal{T}\right|$, and therefore we have an induced homotopy equivalence

$$
\mathrm{B}_{3} \mathcal{T} \simeq|[p] \mapsto| \operatorname{diag} \mathrm{NNN}_{p} \mathcal{T}|| \cong|\operatorname{diag} \mathrm{NNN} \mathcal{T}|
$$

\subsection{The Segal nerve of a tricategory}

Several theoretical interests suggest dealing with the so-called Segal nerve construction for tricategories. This associates to any tricategory $\mathcal{T}$ a simplicial bicategory, denoted by $\mathrm{S} \mathcal{T}$, which can be thought of as a 'rectification' of the pseudosimplicial Grothendieck 
nerve of the tricategory $\mathrm{NT}$, since both are biequivalent in the tricategory of pseudosimplicial bicategories and therefore model the same homotopy type. Furthermore, $\mathrm{S} \mathcal{T}$ is a weak 3-category from the point of view of Tamsamani [46] and Simpson [40] (see Proposition 4.13 below), in the sense that it is a special simplicial bicategory, that is, a simplicial bicategory $\mathrm{S}: \Delta^{\mathrm{op}} \rightarrow$ Hom satisfying the following two conditions:

(i) $\mathrm{S}_{0}$ is discrete (ie all its $1-$ and 2 -cells are identities).

(ii) For $n \geq 2$, the Segal projection homomorphisms below are biequivalences:

$$
p_{n}=\prod_{k=1}^{n} d_{n} \cdots d_{k+1} d_{k-2} \cdots d_{0}: \mathrm{S}_{n} \longrightarrow \mathrm{S}_{1 d_{1}} \times{ }_{d_{0}} \mathrm{~S}_{1 d_{1}} \times d_{0} \cdots d_{1} \times d_{0} \mathrm{~S}_{1}
$$

For a given tricategory $\mathcal{T}$, the construction of the bicategory of unitary homomorphic lax functors from any small category $I$ in the tricategory $\mathcal{T}, I \mapsto \mathbb{L} \operatorname{ax}_{\mathrm{uh}}(I, \mathcal{T})$, given in (6), is functorial on the category $I$, and this leads to the definition below.

Definition 4.12 The Segal nerve of a tricategory $\mathcal{T}$ is the simplicial bicategory

$$
\mathrm{S} \mathcal{T}: \Delta^{\mathrm{op}} \rightarrow \text { Hom } \subset \text { Bicat }, \quad[p] \mapsto \mathrm{S}_{p} \mathcal{T}=\mathbb{L} \operatorname{ax}_{\mathrm{uh}}([p], \mathcal{T})
$$

We should remark that when $\mathcal{T}=\mathcal{B}$ is a bicategory, that is, when its 3 -cells are all identities, then the Segal nerve $\mathrm{SB}$ was introduced by Carrasco, Cegarra and Garzón in [11, Definition 5.2], although it was first studied by Lack and Paoli in [34] under the name of ' 2 -nerve of $\mathcal{B}$ '.

Proposition 4.13 Let $\mathcal{T}$ be a tricategory. Then the following statements hold.

(i) There is a normal pseudosimplicial homomorphism

$$
L: \mathrm{N} \mathcal{T} \rightarrow \mathrm{S} \mathcal{T}
$$

such that for any $p \geq 0$, the homomorphism $L_{p}: \mathrm{N}_{p} \mathcal{T} \rightarrow \mathrm{S}_{p} \mathcal{T}$ is a biequivalence.

(ii) The simplicial bicategory $\mathrm{S} \mathcal{T}$ is special.

Proof We keep the notation established in the construction of $\mathrm{N} \mathcal{T}=(\mathrm{N} \mathcal{T}, \chi, \omega)$ given in the proof of Theorem 3.1, and recall from Lemma 2.4(iii) that we have a biadjoint biequivalence

$$
L_{p} \dashv R_{p}: \mathrm{N}_{p} \mathcal{T} \rightleftarrows \mathrm{S}_{p} \mathcal{T}
$$


The normal pseudosimplicial homomorphism $L=(L, \theta, \Pi): \mathrm{N} \mathcal{T} \rightarrow \mathrm{S} \mathcal{T}$ is then defined by the homomorphisms $L_{p}: \mathrm{N}_{p} \mathcal{T} \rightarrow \mathrm{S}_{p} \mathcal{T}, p \geq 0$. For any $a:[q] \rightarrow[p]$, the structure pseudoequivalence

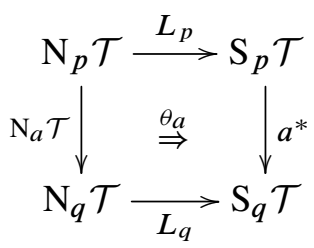

is provided by the counit pseudoequivalence $\mathrm{v}_{q}: L_{q} R_{q} \Rightarrow 1_{\mathrm{S}_{q} \mathcal{T}}$, that is,

$$
L_{q} \mathrm{~N}_{a} \mathcal{T}=L_{q} R_{q} a^{*} L_{p} \stackrel{\theta_{a}=\mathrm{v}_{q} a^{*} L_{p}}{\longrightarrow} a^{*} L_{p}
$$

For any two composable arrows $[n] \stackrel{b}{\longrightarrow}[q] \stackrel{a}{\longrightarrow}[p]$ of $\Delta$, the structure invertible modification

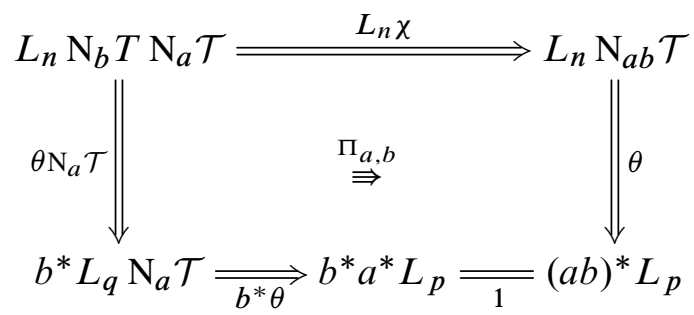

is directly provided by the canonical modification (17), $\Pi_{a, b}=\omega_{b}^{\prime} a^{*} L_{p}$.

The coherence conditions for $L$ (ie [12, Conditions (CC3), (CC4)] with the modifications $1 \circ 1 \cong 1$ of the coherence isomorphisms), are easily verified by using Fact 1.3. This completes the proof of part (i).

When it comes to part (ii), that $\mathrm{S} \mathcal{T}$ is a special simplicial bicategory, we have the isomorphisms

$$
\mathrm{S}_{0} \mathcal{T}=\operatorname{Lax}_{\mathrm{uh}}([0], \mathcal{T}) \cong \mathrm{Ob} \mathcal{T}=\mathrm{N}_{0} \mathcal{T}
$$

between bicategories which identify a normal trihomomorphism $F:[0] \rightarrow \mathcal{T}$ with the object $F 0$,

$$
\mathrm{S}_{1} \mathcal{T}=\mathbb{L} \mathrm{ax}_{\mathrm{uh}}([1], \mathcal{T}) \cong \bigsqcup_{\left(t_{0}, t_{1}\right)} \mathcal{T}\left(t_{0}, t_{1}\right)=\mathrm{N}_{1} \mathcal{T}
$$


which carries a normal trihomomorphism $F:[1] \rightarrow \mathcal{T}$ to the $1-$ cell $F_{0,1}: F 0 \rightarrow F 1$, and, for any integer $p \geq 2$,

$$
\begin{aligned}
\mathrm{S}_{1} \mathcal{T}_{d_{1}} \times d_{0} \cdots & d_{1} \times{ }_{d_{0}} \mathrm{~S}_{1} \mathcal{T} \\
& \cong \bigsqcup_{\left(t_{p-1}, t_{p}\right)} \mathcal{T}\left(t_{p-1}, t_{p}\right) \times \bigsqcup_{\left(t_{p-2}, t_{p-1}\right)} \mathcal{T}\left(t_{p-2}, t_{p-1}\right) \times \cdots \times \bigsqcup_{\left(t_{0}, t_{1}\right)} \mathcal{T}\left(t_{0}, t_{1}\right) \\
& \cong \bigsqcup_{\left(t_{0}, \ldots, t_{p}\right)} \mathcal{T}\left(t_{p-1}, t_{p}\right) \times \mathcal{T}\left(t_{p-2}, t_{p-1}\right) \times \cdots \times \mathcal{T}\left(t_{0}, t_{1}\right)=\mathrm{N}_{p} \mathcal{T}
\end{aligned}
$$

Through these isomorphisms we see that, for any integer $p \geq 2$, the Segal projection homomorphism (18) is precisely the biequivalence $R_{p}: \mathrm{S}_{p} \mathcal{T} \rightarrow \mathrm{N}_{p} \mathcal{T}$ which, recall, is defined by restricting it to the basic graph of the category $[p]$. Whence the simplicial bicategory $\mathrm{S} \mathcal{T}$ is special.

The following theorem states that the classifying space of a tricategory $\mathcal{T}$ can be realized, up to homotopy equivalence, by its Segal nerve $S \mathcal{T}$. This fact will be relevant for our later discussions on loop spaces. Let

$$
\mathrm{B}_{2} \mathrm{ST}: \Delta^{\mathrm{op}} \rightarrow \text { Top, } \quad[p] \mapsto \mathrm{B}_{2} \mathrm{~S}_{p} \mathcal{T}
$$

be the simplicial space obtained by composing $\mathrm{S} \mathcal{T}: \Delta^{\text {op }} \rightarrow$ Hom $\subset$ Bicat with the classifying functor $\mathrm{B}_{2}:$ Hom $\rightarrow$ Top (recall Fact 4.1).

Theorem 4.14 For any tricategory $\mathcal{T}$, there is a homotopy equivalence

$$
\mathrm{B}_{3} \mathcal{T} \simeq\left|\mathrm{B}_{2} \mathrm{~S} \mathcal{T}\right|
$$

Proof Let us consider the pseudosimplicial homomorphism (20), $L: \mathrm{N} \mathcal{T} \rightarrow \mathrm{S} \mathcal{T}$. Since, for every integer $p \geq 0$, the homomorphism $L_{p}: \mathrm{N}_{p} \mathcal{T} \rightarrow \mathrm{S}_{p} \mathcal{T}$ is a biequivalence, it follows from Fact 4.3 that the induced cellular map $\mathrm{B}_{2} L_{p}: \mathrm{B}_{2} \mathrm{~N}_{p} \mathcal{T} \rightarrow \mathrm{B}_{2} \mathrm{~S}_{p} \mathcal{T}$ is a homotopy equivalence. Then by Fact 4.6, the induced map $\mathrm{B}_{2} \int_{\Delta} L: \mathrm{B}_{2} \int_{\Delta} \mathrm{N} \mathcal{T} \rightarrow$ $\mathrm{B}_{2} \int_{\Delta} \mathrm{S} \mathcal{T}$ is a homotopy equivalence. Since $\mathrm{B}_{3} \mathcal{T}=\mathrm{B}_{2} \int_{\Delta} \mathrm{N} \mathcal{T}$ by definition, whereas by Fact 4.7 there is a homotopy equivalence $B_{2} \int_{\Delta} S \mathcal{T} \simeq\left|B_{2} S \mathcal{T}\right|$, the claimed homotopy equivalence follows.

Example 4.15 (Classifying spaces of monoidal bicategories) Any monoidal bicategory $(\mathcal{B}, \otimes)=(\mathcal{B}, \otimes, \mathrm{I}, \boldsymbol{a}, \boldsymbol{l}, \boldsymbol{r}, \pi, \mu, \lambda, \rho)$ can be viewed as a tricategory

$$
\Sigma(\mathcal{B}, \otimes)
$$

with only one object, say $*$, whose hom bicategory is the underlying bicategory. Thus $\Sigma(\mathcal{B}, \otimes)(*, *)=\mathcal{B}$, and its composition given by the tensor functor $\otimes: \mathcal{B} \times \mathcal{B} \rightarrow \mathcal{B}$ 
and the identity at the object is $1_{*}=\mathrm{I}$, the unit object of the monoidal bicategory. The structure pseudoequivalences and modifications $\boldsymbol{a}, \boldsymbol{l}, \boldsymbol{r}, \pi, \mu, \lambda$ and $\rho$ for $\Sigma(\mathcal{B}, \otimes)$ are just those of the monoidal bicategory (see the paper by Cheng and Gurski [15, Section 3] for details). Call this tricategory the suspension, or delooping, tricategory of the bicategory $\mathcal{B}$ induced by the monoidal structure given on it, and call its corresponding Grothendieck nerve the nerve of the monoidal bicategory, hereafter denoted by $\mathrm{N}(\mathcal{B}, \otimes)$. Thus

$$
\mathrm{N}(\mathcal{B}, \otimes)=\mathrm{N} \Sigma(\mathcal{B}, \otimes): \Delta^{\mathrm{op}} \rightarrow \text { Bicat }, \quad[p] \mapsto \mathcal{B}^{p}
$$

is a normal pseudosimplicial bicategory, whose bicategory of $p$-simplices is the $p$ fold power of the underlying bicategory $\mathcal{B}$, with face and degeneracy homomorphisms induced by the tensor homomorphism $\otimes: \mathcal{B} \times \mathcal{B} \rightarrow \mathcal{B}$ and unit object I following the familiar formulas (10) in analogy with those of the reduced bar construction on a topological monoid, and with structure pseudoequivalences and modifications canonically arising from the data of the monoidal structure on $\mathcal{B}$. The general Definition 4.8 for classifying spaces of tricategories leads to the following.

Definition 4.16 The classifying space of the monoidal bicategory, denoted $\mathrm{B}_{3}(\mathcal{B}, \otimes)$, is defined to be the classifying space of its delooping tricategory $\Sigma(\mathcal{B}, \otimes)$. Thus

$$
\mathrm{B}_{3}(\mathcal{B}, \otimes)=\mathrm{B}_{3} \Sigma(\mathcal{B}, \otimes)=\mathrm{B}_{2} \int_{\Delta} \mathrm{N}(\mathcal{B}, \otimes)
$$

The next theorem extends a well known result by Mac Lane and Stasheff on monoidal categories to monoidal bicategories.

Theorem 4.17 Let $(\mathcal{B}, \otimes)$ be a monoidal bicategory such that for any object $x \in \mathcal{B}$, the homomorphism $x \otimes-: \mathcal{B} \rightarrow \mathcal{B}$ induces a homotopy autoequivalence on the classifying space of $\mathcal{B}, \mathrm{B}_{2}(x \otimes-): \mathrm{B}_{2} \mathcal{B} \simeq \mathrm{B}_{2} \mathcal{B}$. Then there is a homotopy equivalence

$$
\mathrm{B}_{2} \mathcal{B} \simeq \Omega \mathrm{B}_{3}(\mathcal{B}, \otimes)
$$

between the classifying space of the underlying bicategory and the loop space of the classifying space of the monoidal bicategory.

Proof By Theorem 4.14, $\mathrm{B}_{3}(\mathcal{B}, \otimes)$ is homotopy equivalent to $\left|X_{\bullet}\right|$, the geometric realization of the simplicial space $X_{\bullet}=\mathrm{B}_{2} \mathrm{~S} \Sigma(\mathcal{B}, \otimes): \Delta^{\mathrm{op}} \rightarrow$ Top obtained by taking classifying spaces on the simplicial bicategory $S \Sigma(\mathcal{B}, \otimes)$, which is the Segal nerve of the suspension tricategory of the monoidal bicategory. By Proposition 4.13, $\mathrm{S} \Sigma(\mathcal{B}, \otimes)$ is a special simplicial bicategory. Furthermore, since the tricategory $\Sigma(\mathcal{B}, \otimes)$ has only one object, the simplicial bicategory $\operatorname{S} \Sigma(\mathcal{B}, \otimes)$ is reduced (see (21)), that is, $\mathrm{S}_{0} \Sigma(\mathcal{B}, \otimes)=*$ is the one-object discrete bicategory. Hence the simplicial space 
$\mathrm{B}_{2} \mathrm{~S} \Sigma(\mathcal{B}, \otimes)$ satisfies [39, Proposition 1.5(i), (ii)]: $X_{0}=\mathrm{B}_{2} \mathrm{~S}_{0} \Sigma(\mathcal{B}, \otimes)$ is contractible, and the Segal projections maps

$$
\mathrm{B}_{2} p_{n}: X_{n}=\mathcal{B S}_{n} \Sigma(\mathcal{B}, \otimes) \rightarrow X_{1}^{n}=\mathrm{B}_{2}\left(\mathrm{~S}_{1} \Sigma(\mathcal{B}, \otimes)^{n}\right) \stackrel{(22)}{\cong} \mathrm{B}_{2} \mathcal{B}^{n}
$$

are homotopy equivalences. Since the $H$-space structure on $X_{1}=\mathrm{B}_{2} \mathcal{B}$ is just induced by tensor homomorphism $\otimes: \mathcal{B} \times \mathcal{B} \rightarrow \mathcal{B}$, we have by hypothesis that $X_{1}$ has homotopy inverses. Therefore, from [39, Proposition 1.5(b)], we can conclude that the canonical map $X_{1} \rightarrow \Omega\left|X_{\bullet}\right|$ is a homotopy equivalence, whence the homotopy equivalence $\mathrm{B}_{2} \mathcal{B} \simeq \Omega \mathrm{B}_{3}(\mathcal{B}, \otimes)$ follows.

Example 4.18 (Classifying spaces of braided monoidal categories) If $(C, \otimes)$ is a monoidal category, then $\Sigma(C, \otimes)$ is the bicategory called by Kapranov and Voevodsky in $[31,2.10]$ the delooping bicategory of the category induced by its monoidal structure. The nerve of $\Sigma(C, \otimes)$ then becomes the pseudosimplicial category used by Jardine in [28, Section 3] to define the classifying space of the monoidal category just as above: $\mathrm{B}_{2}(C, \otimes)=\mathrm{B} \int_{\Delta} \mathrm{N}(C, \otimes)$ (see also Bullejos and Cegarra [8], or Balteanu et al [3]). Thus

$$
\mathrm{B}_{2}(C, \otimes)=\mathrm{B}_{2} \Sigma(C, \otimes) .
$$

Now let $(C, \otimes, c)$ be a braided monoidal category as defined by Joyal and Street in [29, Definition 3.1]. Thanks to the braidings $c: x \otimes y \rightarrow y \otimes x$, the given tensor product on $C$ defines in a natural way a tensor product homomorphism on the suspension bicategory of the underlying monoidal category, $\otimes: \Sigma(C, \otimes) \times \Sigma(C, \otimes) \rightarrow \Sigma(C, \otimes)$. Thus $(\Sigma(C, \otimes), \otimes)$ is a monoidal bicategory. The corresponding suspension tricategory

$$
\Sigma^{2}(C, \otimes, c)=\Sigma(\Sigma(C, \otimes), \otimes)
$$

is called the double suspension, or double delooping, of the underlying category $C$ associated to the given braided monoidal structure on it (see Berger [5, 4,2.5], Kapranov and Voevodsky [31, 4.2] or Gordon, Power and Street [20, 7.9]). We refer the reader to Cheng and Gurski [15, Section 2] for the (nontrivial details) of this construction and recall that this is a tricategory with only one object, say $*$, only one arrow $*=$ $1_{*}: * \rightarrow *$, the objects of $C$ are the 2-cells, and the morphisms of $C$ are the 3-cells. The hom bicategory is $\Sigma^{2}(C, \otimes, c)(*, *)=\Sigma(C, \otimes)$, the suspension bicategory of the underlying monoidal category $(C, \otimes)$, the composition is also (as the horizontal one in $\Sigma(C, \otimes))$ given by the tensor functor $\otimes: C \times C \rightarrow C$ and the interchange 3-cell between the two different composites of 2 -cells is given by the braiding.

The most striking instance is for $(C, \otimes, c)=(A,+, 0)$, the strict braided monoidal category with only one object defined by an abelian group $A$, where both composition 
and tensor product are given by the addition + in $A$; in this case, the double suspension tricategory $\Sigma^{2} A$ is precisely the 3-category treated in Examples 2.1 and 2.2.

For any braided monoidal category $(C, \otimes, c)$, the Grothendieck nerve of the double suspension tricategory $\Sigma^{2}(C, \otimes, c)$ coincides with the pseudosimplicial bicategory called by Carrasco, Cegarra and Garzón in [12] the nerve of the braided monoidal category, and denoted by $\mathrm{N}(C, \otimes, c)$. Thus

$$
\mathrm{N}(C, \otimes, c)=\mathrm{N} \Sigma^{2}(C, \otimes, c): \Delta^{\mathrm{op}} \rightarrow \text { Bicat }, \quad[p] \mapsto(\Sigma(C, \otimes))^{p}=\Sigma\left(C^{p}, \otimes\right)
$$

is a normal pseudosimplicial one-object bicategory whose bicategory of $p$-simplices is the suspension bicategory of the monoidal category $p$-fold power of $(C, \otimes)$. Since the classifying space of the braided monoidal category [12, Definition 6.1$], \mathrm{B}_{3}(C, \otimes, c)$, is just given by

$$
\mathrm{B}_{3}(C, \otimes, c)=\mathrm{B}_{2} \int_{\Delta} \mathrm{N}(C, \otimes, c)
$$

we have the following.

Corollary 4.19 The classifying space of a braided monoidal category is the classifying space of its double suspension tricategory, that is, $\mathrm{B}_{3}(C, \otimes, c)=\mathrm{B}_{3} \Sigma^{2}(C, \otimes, c)$.

It is known that the group completion of the classifying space $\mathrm{BC}$ of a braided monoidal category $(C, \otimes, c)$ is a double loop space. This fact was first noticed by Stasheff in [41], but originally proven by Fiedorowicz in [17] (other proofs can be found in [3] or Berger [5]). We shall show below that under favorable circumstances $\mathrm{B}_{3}(C, \otimes, c)$ is a model for a such double delooping of $\mathrm{B} C$.

Corollary 4.20 (i) For any braided monoidal category $(C, \otimes, c)$ there is a homotopy equivalence

$$
\mathrm{B}_{2}(C, \otimes) \simeq \Omega_{3}(C, \otimes, c) .
$$

(ii) Let $(C, \otimes, c)$ be a braided monoidal category such that for any object $x \in C$, the functor $x \otimes-: C \rightarrow C$ induces a homotopy autoequivalence on the classifying space of $C, \mathcal{B}(x \otimes-): \mathrm{B} C \simeq \mathrm{B} C$. Then there is a homotopy equivalence

$$
\mathrm{B} C \simeq \Omega^{2} \mathrm{~B}_{3}(C, \otimes, c)
$$

between the classifying space of the underlying category and the double loop space of the classifying space of underlaying category.

Proof (i) By Corollary 4.19 we have that the classifying space of any braided monoidal category $(C, \otimes, c)$ is the same as the classifying space of the monoidal bicategory $\Sigma(\Sigma(C, \otimes), \otimes)$. Therefore $\Omega \mathrm{B}_{3}(C, \otimes, c)=\Omega_{\mathrm{B}_{3}} \Sigma(\Sigma(C, \otimes), \otimes)$. Since 
$\Sigma(C, \otimes)$ has only one object, it is obvious that its monoid of connected component $\pi_{0} \Sigma(C, \otimes)$ is the trivial group 1 . Then by Theorem 4.17 , there is a homotopy equivalence $\mathrm{B}_{2} \Sigma(C, \otimes) \simeq \Omega \mathrm{B}_{3} \Sigma(\Sigma(C, \otimes), \otimes)$. Since by $(24), \mathrm{B}_{2}(C, \otimes)=\mathrm{B}_{2} \Sigma(C, \otimes)$, the result follows.

(ii) From the discussion in Example 4.9, we have homotopy equivalences $\mathrm{BC} \simeq \mathrm{B}_{2} C$ and $\mathrm{B}_{2}(C, \otimes) \simeq \mathrm{B}_{3}(C, \otimes)$. Then by Theorem 4.17 , there is a homotopy equivalence $\mathrm{B} C \simeq \Omega \mathrm{B}_{2}(C, \otimes)$. By the already proved (i), there is a homotopy equivalence $\Omega \mathrm{B}_{2}(C, \otimes) \simeq \Omega^{2} \mathrm{~B}_{3}(C, \otimes, c)$, whence the result.

\section{The geometric nerve of a tricategory}

With the notion of the classifying space of a tricategory $\mathcal{T}$ given above, the resulting $\mathrm{CW}$-complex $\mathcal{B} \mathcal{T}$ thus obtained has many cells with little apparent intuitive connection with the cells of the original tricategory, and they do not enjoy any proper geometric meaning. This leads one to search for any simplicial set realizing the space $\mathrm{B}_{3} \mathcal{T}$ whose cells give a logical geometric meaning to the data of the tricategory. With the definition below we give a natural candidate for such a simplicial set, which, up to minor changes affecting the direction conventions on 2- and 3-cells, is essentially due to Street [43; 45].

For any given tricategory $\mathcal{T}$, the construction $I \mapsto \operatorname{Lax}_{\mathrm{u}}(I, \mathcal{T})$ given in (2), which carries each category $I$ to the set unitary lax functors from $I$ into $\mathcal{T}$, is clearly functorial on the small category $I$, whence the following simplicial set.

Definition 5.1 The geometric nerve of a tricategory $\mathcal{T}$ is the simplicial set

$$
\Delta \mathcal{T}: \Delta^{\text {op }} \rightarrow \text { Set }, \quad[p] \mapsto \operatorname{Lax}_{\mathrm{u}}([p], \mathcal{T}) .
$$

The simplicial set $\Delta \mathcal{T}$ encodes the entire tricategorical structure of $\mathcal{T}$ and, as we will prove below, represents the classifying space of the tricategory $\mathcal{T}$ up to homotopy. We shall stress here that the simplices of the geometric nerve $\Delta \mathcal{T}$ have the following pleasing geometric description, where we have taken into account the coherence theorem for tricategories in order to interpret correctly the pasting diagrams (ie by thinking of $\mathcal{T}$ as a Gray-category). The vertices of $\Delta \mathcal{T}$ are points labelled with the objects $F 0$ of $\mathcal{T}$. The 1-simplices are paths labelled with the 1 -cells

$$
F_{0,1}: F 0 \rightarrow F 1
$$


The 2-simplices are oriented triangles

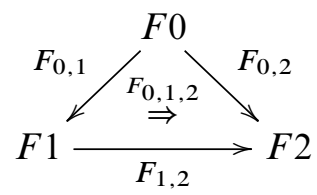

with objects $F i$ placed on the vertices, 1-cells $F_{i, j}: F i \rightarrow F j$ on the edges and labelling the inner as a 2-cell $F_{0,1,2}: F_{1,2} \otimes F_{0,1} \Rightarrow F_{0,2}$. For $p \geq 3$, a $p$-simplex of $\Delta \mathcal{T}$ is geometrically represented by a diagram in $\mathcal{T}$ with the shape of the 3 -skeleton of an oriented standard $p$-simplex whose 3 -faces are oriented tetrahedrons:

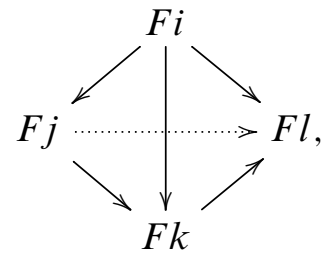

There is one for each $0 \leq i<j<k<l \leq p$, whose faces
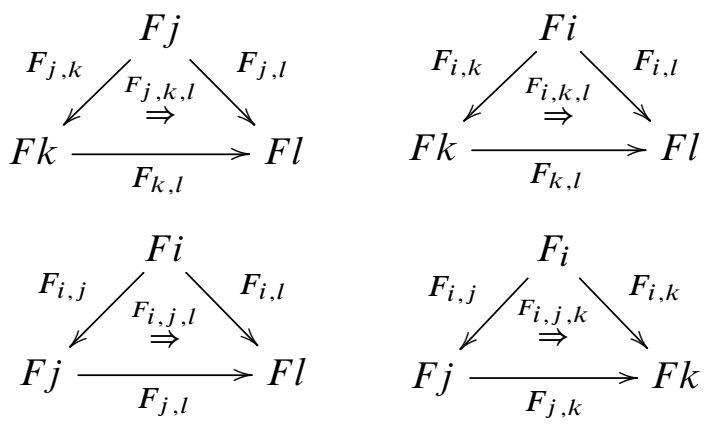

are geometric 2-simplices as above, and
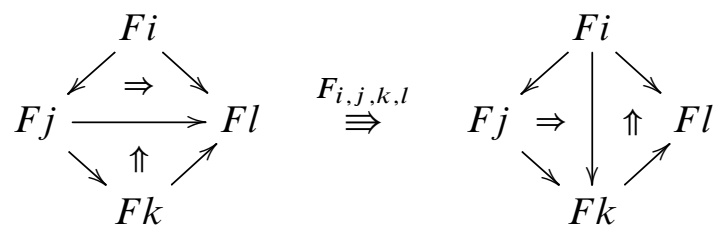

is a 3-cell of the tricategory that labels the inner of the tetrahedron. For $p \geq 4$, these data are required to satisfy the coherence condition (CR1) as stated in the Appendix; 
that is, for each $0 \leq i<j<k<l<m \leq p$, the following diagram commutes:

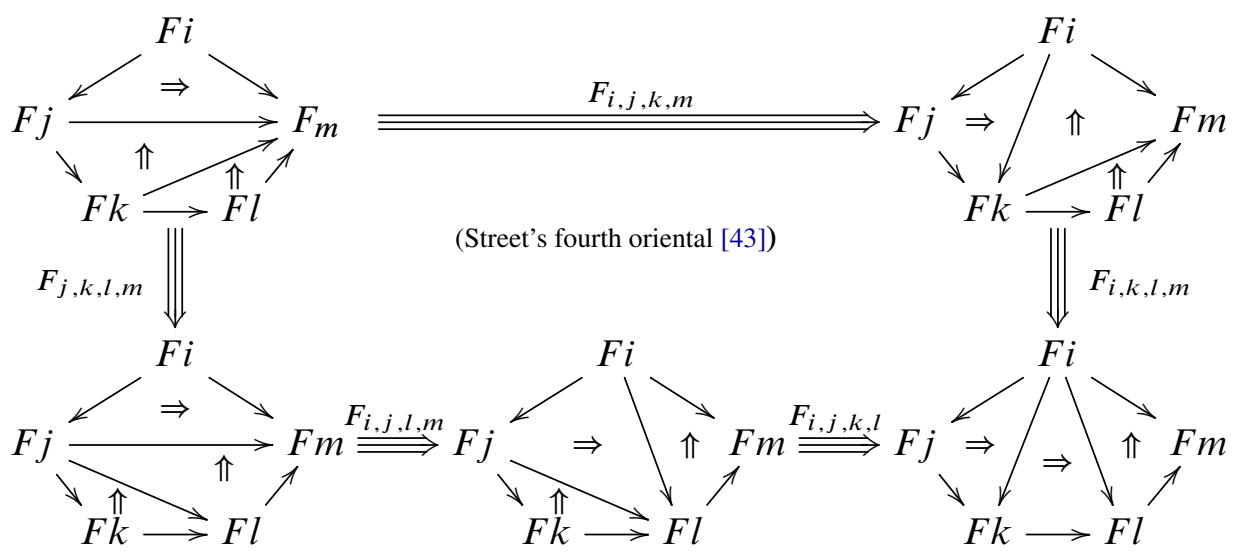

The simplicial set $\Delta \mathcal{T}$ is coskeletal in dimensions greater than 4. More precisely, for $p \geq 4$, a $p$-simplex $F: \Delta[p] \rightarrow \mathcal{T}$ of $\Delta \mathcal{T}$ is determined uniquely by its boundary $\partial F=\left(F d^{0}, \ldots, F d^{p}\right)$

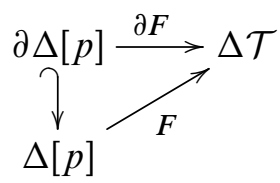

and, for $p \geq 5$, every possible boundary of a $p$-simplex, $\partial \Delta[p] \rightarrow \Delta \mathcal{T}$, is actually the boundary $\partial F$ of a geometric $p$-simplex $F$ of the tricategory $\mathcal{T}$.

Example 5.2 (Geometric nerves of bicategories) When a bicategory $\mathcal{B}$ is regarded as a tricategory, all of whose 3-cells are identities, then the simplicial set $\Delta \mathcal{B}$ is precisely the unitary geometric nerve of the bicategory, as it is called by Carrasco, Cegarra and Garzón in [11] (but denoted by $\Delta^{\mathrm{u}} \mathcal{B}$ ). The construction of the geometric nerve for a bicategory was first given in the late eighties by Duskin and Street (see [44, page 573]). In [16], Duskin gave a characterization of the unitary geometric nerve of a bicategory $\mathcal{B}$ in terms of its simplicial structure. The result states that a simplicial set is isomorphic to the geometric nerve of a bicategory if and only if it satisfies the coskeletal conditions above as well as supporting appropriate sets of 'abstractly invertible' 1-and 2-simplices (see Gurski [22] for an interesting new approach to this subject). In [11, Theorem 6.1], the following fact is proved.

Fact 5.3 For any bicategory $\mathcal{B}$, there is a homotopy equivalence $\mathrm{B}_{2} \mathcal{B} \simeq|\Delta \mathcal{B}|$.

We now state a main result of the paper. 
Theorem 5.4 For any tricategory $\mathcal{T}$, there is a homotopy equivalence $\mathrm{B}_{3} \mathcal{T} \simeq|\Delta \mathcal{T}|$.

Proof Let us consider for any given tricategory $\mathcal{T}$, the simplicial bicategory

$$
\underline{\mathcal{T}}: \Delta^{\mathrm{op}} \rightarrow \text { Hom } \subset \text { Bicat }, \quad[q] \mapsto \mathbb{L a x}_{\mathrm{u}}([q], \mathcal{T})
$$

whose bicategories of $q$-simplices are the bicategories of unitary lax functors (5) of $[q]$ into $\mathcal{T}$. In this simplicial bicategory, the homomorphism induced by any map $a:[q] \rightarrow[p], a^{*}: \underline{\Delta}_{p} \mathcal{T} \rightarrow \underline{\Delta}_{q} \mathcal{T}$ is actually a strict functor. Hence the bisimplicial set

$$
\Delta \underline{\Delta} \mathcal{T}: \Delta^{\mathrm{op}} \times \Delta^{\mathrm{op}} \rightarrow \text { Set }, \quad([p],[q]) \mapsto \Delta_{p} \underline{\Delta}_{q} \mathcal{T}=\operatorname{Lax}_{\mathrm{u}}\left([p], \mathbb{L} \operatorname{Lax}_{\mathrm{u}}([q], \mathcal{T})\right)
$$

is well-defined, since the geometric nerve construction $\Delta$ is functorial on unitary homomorphisms between bicategories. The plan is to prove the existence of homotopy equivalences

$$
\begin{aligned}
\mathrm{B}_{3} \mathcal{T} & \simeq|\operatorname{diag} \Delta \underline{\Delta \mathcal{T}}|, \\
|\Delta \mathcal{T}| & \simeq|\operatorname{diag} \Delta \underline{\Delta} \mathcal{T}|,
\end{aligned}
$$

from which the theorem follows.

The homotopy equivalence (30) the Segal nerve of the tricategory (19) is a simplicial sub-bicategory of $\underline{\Delta} \mathcal{T}$. Let $L: \mathrm{N} \mathcal{T} \rightarrow \underline{\mathcal{T}}$ be the pseudosimplicial homomorphism obtained by composing the pseudosimplicial homomorphism (20), equally denoted by $L: \mathrm{N} \mathcal{T} \rightarrow \mathrm{S} \mathcal{T}$, with the simplicial inclusion $\mathrm{S} \mathcal{T} \subseteq \underline{\Delta} \mathcal{T}$. At any degree $p \geq 0$, the homomorphism $L_{p}: \mathrm{N}_{p} \mathcal{T} \rightarrow \underline{\Delta}_{p} \mathcal{T}$ is precisely the homomorphism in Lemma 2.4, $\operatorname{Lax}\left(\mathcal{G}_{p}, \mathcal{T}\right) \rightarrow \operatorname{Lax}_{\mathrm{u}}([p], \mathcal{T})$, corresponding with the basic graph of the category $[p]$. Then, we have a homomorphism $R_{p}: \underline{\Delta}_{p} \mathcal{T} \rightarrow \mathrm{N}_{p} \mathcal{T}$ such that $R_{p} L_{p}=1_{\mathrm{N}_{p}} \mathcal{T}$, and a lax transformation $\mathrm{v}_{p}: L_{p} R_{p} \Rightarrow 1_{\underline{\Delta}_{p}} \mathcal{T}$. It follows from Fact 4.2 that every induced map $\mathrm{B}_{2} L_{p}: \mathrm{B}_{2} \mathrm{~N}_{p} \mathcal{T} \rightarrow \mathrm{B}_{2} \underline{\Delta}_{p} \mathcal{T}$ is a homotopy equivalence. Then, by Fact 4.6 , the induced map $\mathrm{B}_{2} \int_{\Delta} L: \mathrm{B}_{2} \int_{\Delta} \mathrm{N} \mathcal{T} \rightarrow \mathrm{B}_{2} \int_{\Delta} \Delta \mathcal{T}$ is a homotopy equivalence. Let

$$
\mathrm{B}_{2} \underline{\Delta} \mathcal{T}: \Delta^{\mathrm{op}} \rightarrow \text { Top, } \quad[p] \mapsto \mathrm{B}_{2} \underline{\Delta}_{p} \mathcal{T}
$$

be the simplicial space obtained by composing $\underline{\mathcal{T}}$ with the classifying functor $\mathcal{B}:$ Hom $\rightarrow$ Top (see Fact 4.1). Since, by definition, $\mathrm{B}_{3} \mathcal{T}=\mathrm{B}_{2} \int_{\Delta} \mathrm{N} \mathcal{T}$, whereas, by Fact 4.7, there is a homotopy equivalence $\mathrm{B}_{2} \int_{\Delta} \underline{\mathcal{T}} \simeq\left|\mathrm{B}_{2} \underline{\Delta} \mathcal{T}\right|$, we have a homotopy equivalence $\mathrm{B}_{3} \mathcal{T} \simeq\left|\mathrm{B}_{2} \underline{\Delta} \mathcal{T}\right|$. Furthermore, by Fact 5.3, we have a homotopy equivalence

$$
\left|\mathrm{B}_{2} \underline{\Delta} \mathcal{T}\right|=\left|[q] \mapsto \mathrm{B}_{2} \underline{\Delta}_{q} \mathcal{T}\right| \simeq|[q] \mapsto| \Delta \underline{\Delta}_{q} \mathcal{T}|| \cong|\operatorname{diag} \Delta \underline{\Delta} \mathcal{T}|,
$$

where we refer to Quillen's Lemma [37, page 86] for the last homeomorphism. Thus $\mathcal{B} \mathcal{T} \simeq|\operatorname{diag} \Delta \underline{\Delta} \mathcal{T}|$, as claimed. 
The homotopy equivalence (31) Note that the geometric nerve $\Delta \mathcal{T}$ is the simplicial set of objects of the simplicial bicategory $\underline{\mathcal{T}}$, that is, $\Delta \mathcal{T}=\Delta_{0} \underline{\mathcal{T}}$. Therefore, if we regard $\Delta \mathcal{T}$ as a simplicial discrete bicategory (ie all $1-$ cells and 2-cells are identities), then $\Delta \Delta \mathcal{T}$ becomes a bisimplicial set that is constant in the horizontal direction, and there is a natural bisimplicial map $\Delta \Delta \mathcal{T} \hookrightarrow \Delta \underline{\Delta} \mathcal{T}$, which is, at each horizontal level $p \geq 1$, the composite simplicial map

$$
\Delta \mathcal{T}=\Delta_{0} \underline{\Delta} \mathcal{T} \stackrel{s_{0}^{\mathrm{h}}}{\hookrightarrow} \Delta_{1} \underline{\Delta} \mathcal{T} \hookrightarrow \cdots \hookrightarrow \Delta_{p-1} \Delta \mathcal{T} \stackrel{s_{p-1}^{\mathrm{h}}}{\hookrightarrow} \Delta_{p} \Delta \mathcal{T} .
$$

Next we prove that the simplicial map $\Delta \mathcal{T} \rightarrow \operatorname{diag} \Delta \underline{\Delta T}$, induced on diagonals, is a weak homotopy equivalence, whence the announced homotopy equivalence in (31). It suffices to prove that every one of the simplicial maps in (32) is a weak homotopy equivalence and, in fact, we will prove more: every simplicial $m a p s_{p-1}^{\mathrm{h}}$, $p \geq 1$, embeds the simplicial set $\Delta_{p-1} \underline{\mathcal{T}}$ into $\Delta_{p} \underline{\underline{T}}$ as a simplicial deformation retract. Since we have that $d_{p}^{\mathrm{h}} s_{p-1}^{\mathrm{h}}=1$, it is enough to exhibit a simplicial homotopy $h: 1 \Rightarrow s_{p-1}^{\mathrm{h}} d_{p}^{\mathrm{h}}: \Delta_{p} \underline{\Delta} \mathcal{T} \rightarrow \Delta_{p} \underline{\Delta} \mathcal{T}$.

To do so, we shall use the following notation for the bisimplices in $\Delta \underline{\Delta} \mathcal{T}$. Since such a bisimplex of bidegree $(p, q)$, say $F \in \Delta_{p} \Delta_{q} \mathcal{T}$, is a unitary lax functor of the category $[p]$ in the bicategory of unitary lax functors $\operatorname{Lax}_{\mathrm{u}}([q], \mathcal{T})$, it consists of

- unitary lax functors $F^{u}:[q] \rightarrow \mathcal{T}$ for $0 \leq u \leq p$

- 1-cells $F^{u, v}: F^{u} \Rightarrow F^{v}$ for $0 \leq u<v \leq p$

- 2-cells $F^{u, v, w}: F^{v, w} \circ F^{u, v} \Rightarrow F^{u, w}$ for $0 \leq u<v<w \leq p$

such that the diagrams

$$
\begin{aligned}
& \left(F^{w, t} \circ F^{v, w}\right) \circ F^{u, v} \Longrightarrow \quad \stackrel{a}{\Longrightarrow} F^{w, t} \circ\left(F^{v, w} \circ F^{u, v}\right)
\end{aligned}
$$

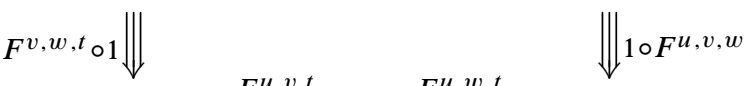

$$
\begin{aligned}
& F^{v, t} \circ F^{u, v} \stackrel{F^{u, v, t}}{=} F^{u, t} \stackrel{F^{u, w, t}}{\Longleftrightarrow} F^{w, t} \circ F^{u, w}
\end{aligned}
$$

commute for $u<v<w<t$. Hence such a $(p, q)-$ simplex is described by a list of cells of the tricategory $\mathcal{T}$

$$
F=\left(F i, F_{i, j}^{u}, F_{i, j, k}^{u}, F_{i, j, k, l}^{u}, F_{i, j}^{u, v}, F_{i, j, k}^{u, v}, F_{i, j}^{u, v, w}\right),
$$

with $0 \leq i<j<k<l \leq q$, where:

- $F i\left(=F^{0} i\right)$ is an object of $\mathcal{T}$.

- $F_{i, j}^{u}: F i \rightarrow F j$ are $1-$ cells in $\mathcal{T}$.

- $F_{i, j, k}^{u}: F_{j, k}^{u} \otimes F_{i, j}^{u} \Rightarrow F_{i, k}^{u}$ are 2-cells in $\mathcal{T}$.

- $F_{i, j}^{u, v}: F_{i, j}^{u} \Rightarrow F_{i, j}^{v}$ are 2-cells in $\mathcal{T}$. 
- $F_{i, j, k, l}^{u}, F_{i, j, k}^{u, v}$ and $F_{i, j}^{u, v, w}$ are 3 -cells in $\mathcal{T}$ of the form

$$
\begin{aligned}
& \left(F_{k, l}^{u} \otimes F_{j, k}^{u}\right) \otimes F_{i, j}^{u} \stackrel{a}{=} F_{k, l}^{u} \otimes\left(F_{j, k}^{u} \otimes F_{i, j}^{u}\right) \\
& F_{j, k, l}^{u} \otimes 1\left\|\stackrel{F_{i, j, k, l}^{u}}{\Rightarrow}\right\| 1 \otimes F_{i, j, k}^{u}
\end{aligned}
$$

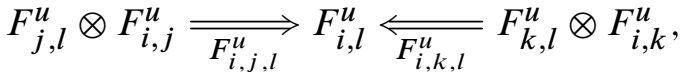

$$
\begin{aligned}
& F_{j, k}^{u} \otimes F_{i, j}^{u} \stackrel{F_{i, j, k}^{u}}{\longrightarrow} F_{i, k}^{u} \\
& F_{j, k}^{u, v} \otimes F_{i, j}^{u, v}\left\|\quad \stackrel{F_{i, j, k}^{u, v}}{\Rightarrow}\right\| F_{i, k}^{u, v} \\
& F_{j, k}^{v} \otimes F_{i, j}^{v} \underset{F_{i, j, k}^{v}}{\Longrightarrow} F_{i, k}^{v}
\end{aligned}
$$

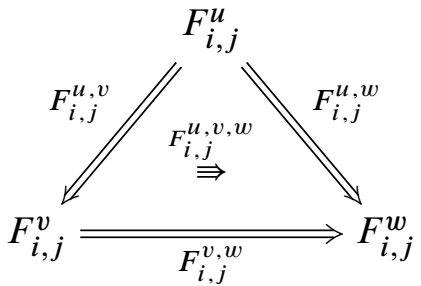

satisfying the various conditions.

The horizontal faces and degeneracies of such a bisimplex (33) are given by the simple rules $d_{m}^{\mathrm{h}} F=\left(F i, F_{i, j}^{d^{m} u}, \ldots\right)$ and $s_{m}^{\mathrm{h}} F=\left(F i, F_{i, j}^{s^{m} u}, \ldots\right)$, whereas the vertical ones are given by

$$
d_{m}^{\mathrm{v}} F=\left(F d^{m_{i}} i, F_{d^{m_{i, d^{m}}}}^{u}, \ldots\right) \quad \text { and } \quad s_{m}^{\mathrm{v}} F=\left(F s^{m_{i}}, F_{s^{m_{i, s^{m}}}}^{u}, \ldots\right) .
$$

We have the simplicial homotopy

$$
h: 1 \Rightarrow s_{p-1}^{\mathrm{h}} d_{p}^{\mathrm{h}}: \Delta_{p} \underline{\Delta} \mathcal{T} \rightarrow \Delta_{p} \underline{\Delta} \mathcal{T} .
$$

For each $0 \leq m \leq q$, the map $h_{m}: \Delta_{p} \underline{\Delta}_{q} \mathcal{T} \rightarrow \Delta_{p} \underline{\Delta}_{q+1} \mathcal{T}$ takes a $(p, q)$-simplex $F$ as in (33) of $\Delta \underline{\Delta} \mathcal{T}$ to the $(p, q+1)$-simplex $h_{m} F$ consisting of the following:

- The lax functors $h_{m} F^{u}=\left(s^{m}\right)^{*} F^{u}:[q+1] \rightarrow \mathcal{T}$ for $0 \leq u<p$

- The lax functors $h_{m} F^{p}:[q+1] \rightarrow \mathcal{T}$ with 


$$
\begin{aligned}
& \left(h_{m} F\right)^{p} i=F s^{m} i \text { for } 0 \leq i \leq q+1 \\
& \left(h_{m} F\right)_{i, j}^{p}= \begin{cases}F_{s^{m}}^{p-1, s^{m} j} & \text { if } j \leq m \\
F_{s^{m}}^{p}{ }_{i, j-1} & \text { if } m<j\end{cases}
\end{aligned}
$$

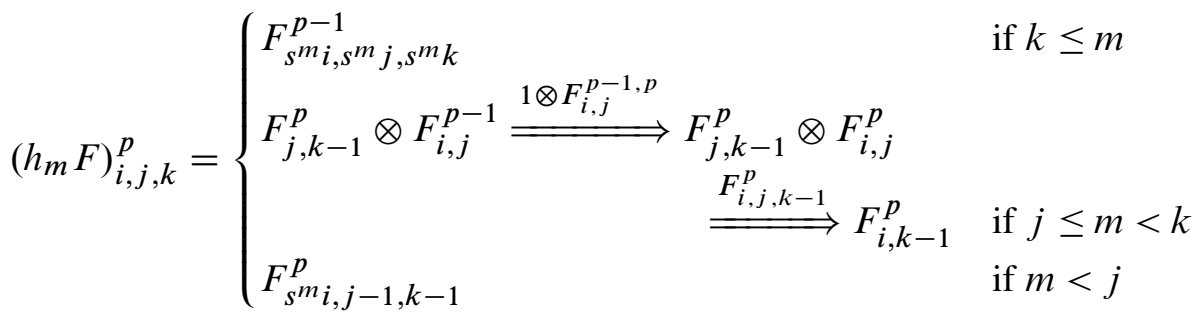

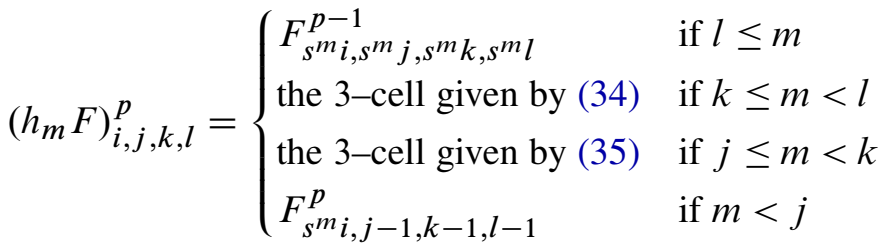

where

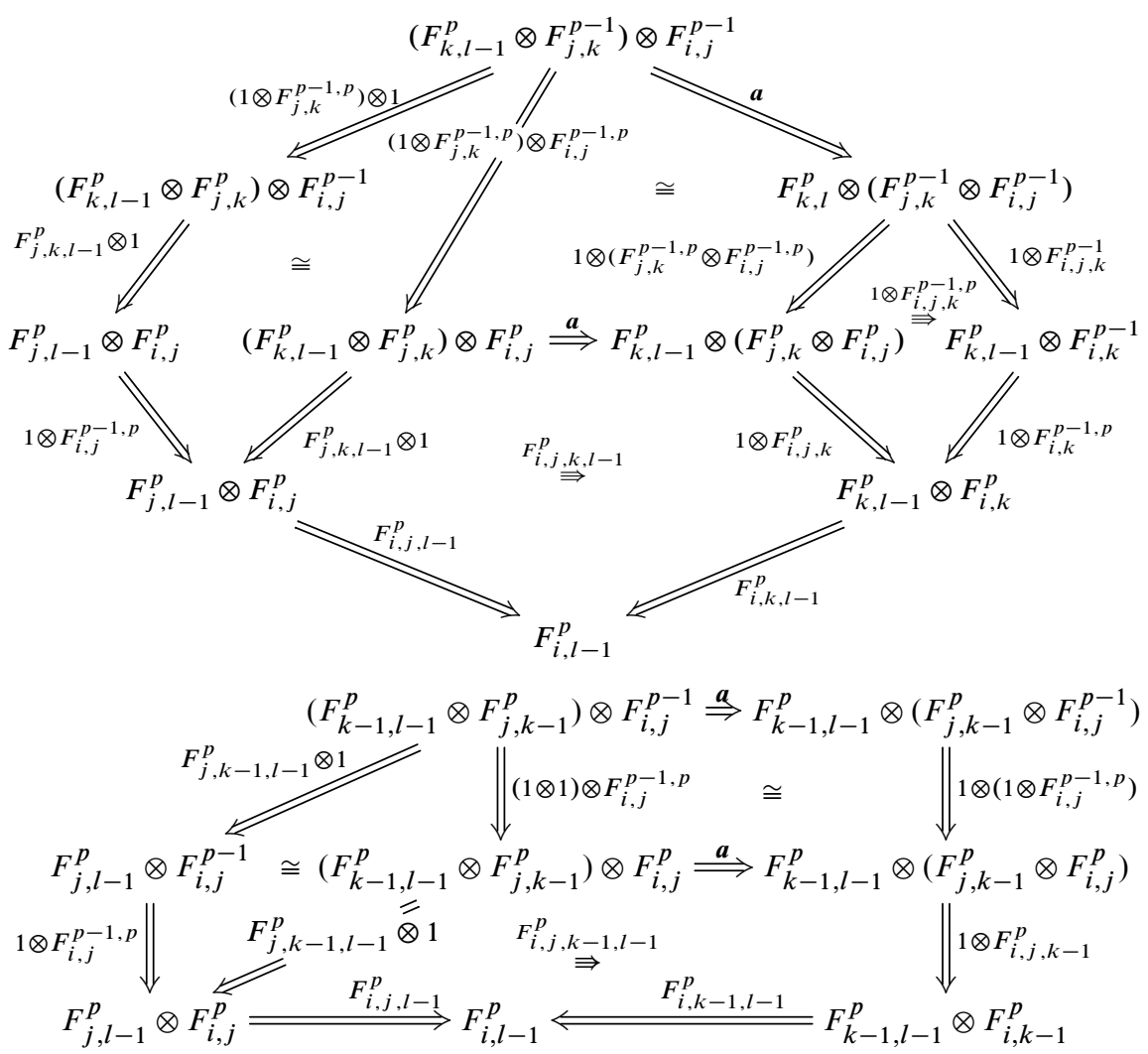


- The 1-cells $h_{m} F^{u, v}=\left(s^{m}\right)^{*} F^{u, v}: h_{m} F^{u} \Rightarrow h_{m} F^{v}$ for $0 \leq u<v<p$

- The 1-cells $h_{m} F^{u, p}: h_{m} F^{u} \Rightarrow h_{m} F^{p}$ with

$$
\begin{aligned}
& -\left(h_{m} F\right)_{i, j}^{u, p}= \begin{cases}F_{s^{m} i, s^{m} j}^{u, p-1} & \text { if } j \leq m \\
F_{s^{m}{ }_{i, j-1}}^{u, j} & \text { if } j>m\end{cases} \\
& -\left(h_{m} F\right)_{i, j, k}^{u, p}= \begin{cases}F_{s^{m} i, s^{m} j, s^{m} k}^{u, p-1} & \text { if } k \leq m \\
\text { the 3-cell given by (36) } & \text { if } j \leq m<k \\
F_{s^{m} i, j-1, k-1}^{u, p} & \text { if } m<j\end{cases}
\end{aligned}
$$

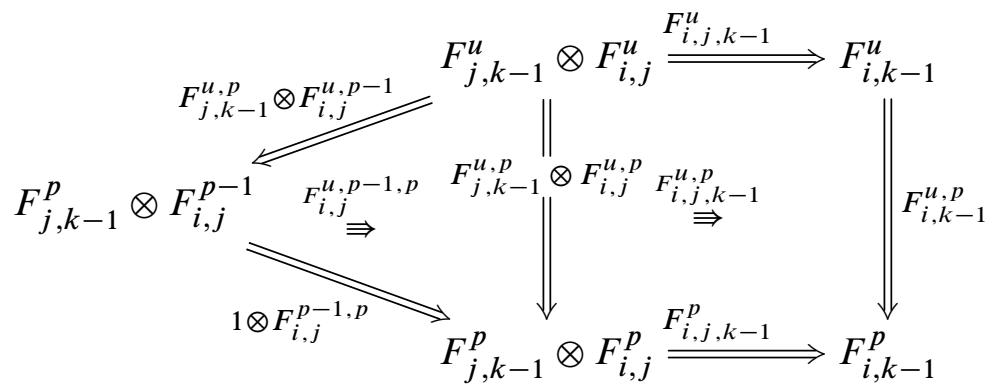

- The 2-cells $\left(h_{m} F\right)^{u, v, w}=\left(s^{m}\right)^{*} F^{u, v, w}$ for $w<p$

- The 2-cells $\left(h_{m} F\right)^{u, v, p}$ are given by $\left(h_{m} F\right)_{i, j}^{u, v, p}= \begin{cases}F_{s^{m} i, s^{m} j}^{u, v, p} & \text { if } j \leq m \\ F_{s^{m} i, j-1}^{u, v} & \text { if } m<j\end{cases}$

So defined, a straightforward (though tedious) verification shows that $h: 1 \Rightarrow s_{p-1}^{\mathrm{h}} d_{p}^{\mathrm{h}}$ is actually a simplicial homotopy, and this completes the proof.

As an application of the theorem above, we shall prove that tritransformations produce homotopies. To do so, the following lemma is the key.

Lemma 5.5 Suppose $\theta=(\theta, \Pi, \mathrm{M}): F \Rightarrow G: \mathcal{T} \rightarrow \mathcal{T}^{\prime}$ is a tritransformation. There is a trihomomorphism $H: \mathcal{T} \times[1] \rightarrow \mathcal{T}^{\prime}$ making this diagram commutative:

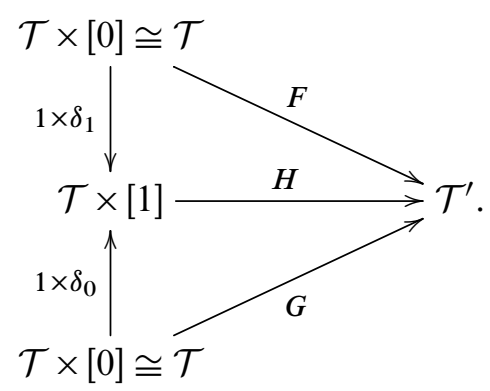


Proof For any objects $x, y$ of $\mathcal{T}, H:(\mathcal{T} \times[1])((x, 0),(y, 1)) \rightarrow \mathcal{T}^{\prime}(F x, G y)$ is the homomorphism composite of

$$
\mathcal{T}(x, y) \times\{(0,1)\} \cong \mathcal{T}(x, y) \stackrel{G}{\rightarrow} \mathcal{T}^{\prime}(G x, G y) \stackrel{\mathcal{T}^{\prime}(\theta x, 1)}{\longrightarrow} \mathcal{T}^{\prime}(F x, G y)
$$

For objects $x, y, z$ of $\mathcal{T}$, the pseudoequivalence

$$
\begin{aligned}
&(\mathcal{T} \times[1])((y, 1),(z, 1)) \times(\mathcal{T} \times[1])((x, 0),(y, 1)) \stackrel{H \times H}{\longrightarrow} \mathcal{T}^{\prime}(G y, G z) \times \mathcal{T}^{\prime}(F x, G y) \downarrow_{\Downarrow x^{H}}^{\otimes} \\
&(\mathcal{T} \times[1])((x, 0),(z, 1)) \longrightarrow \mathcal{T}^{\prime}(F x, G z)
\end{aligned}
$$

is obtained by pasting the diagram

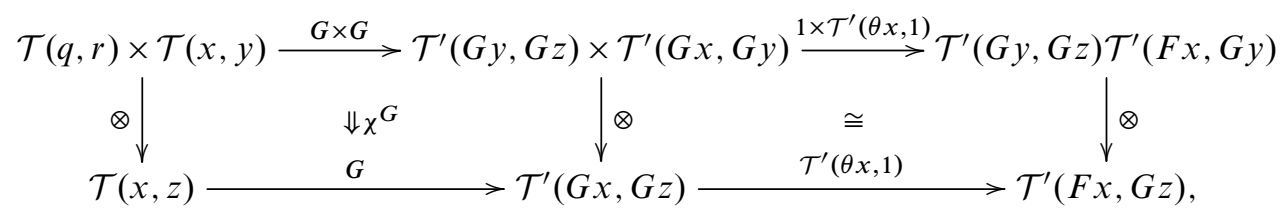

and the pseudoequivalence

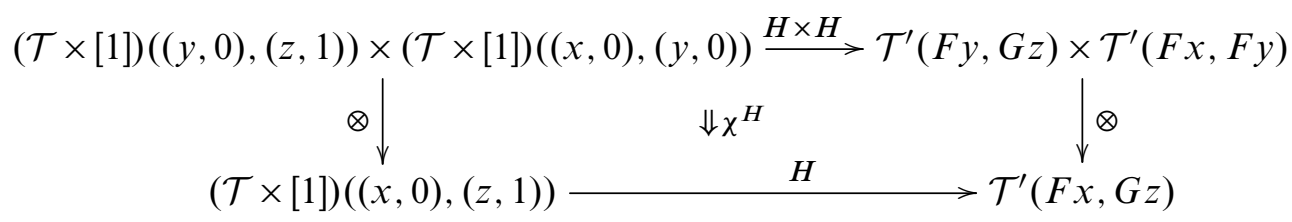

by pasting in

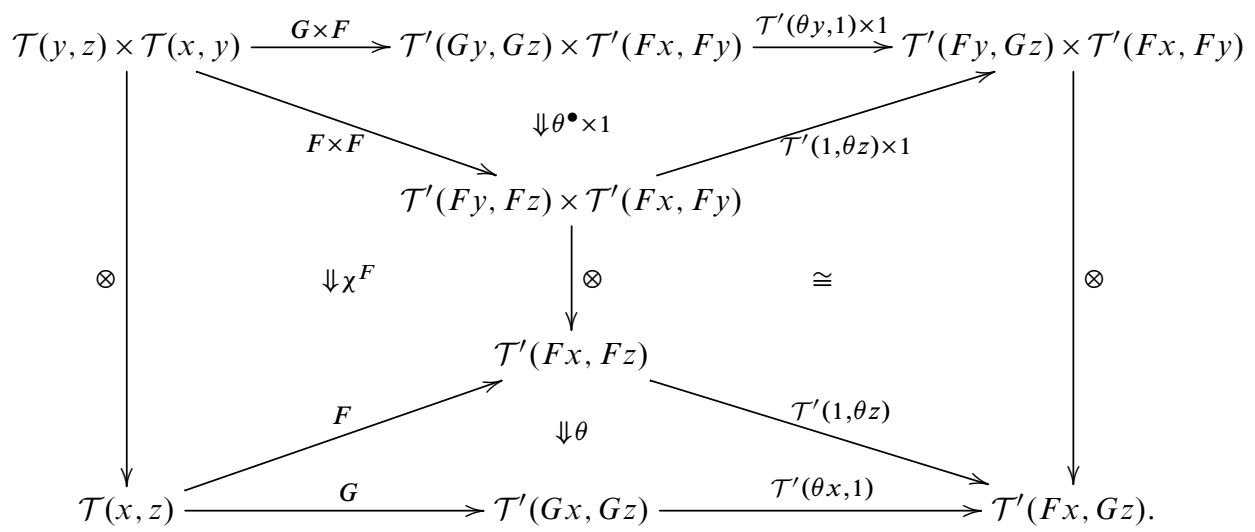


For any objects $x, y, z, t$ of $\mathcal{T}$, the components of the invertible modification $\omega^{H}$ at the triples

$$
\begin{aligned}
& (x, 0) \stackrel{(a,(0,1))}{\longrightarrow}(y, 1) \stackrel{\left(b, 1_{1}\right)}{\longrightarrow}(z, 1) \stackrel{\left(c, 1_{1}\right)}{\longrightarrow}(t, 1), \\
& (x, 0) \stackrel{\left.\left(a, 1_{0}\right)\right)}{\longrightarrow}(y, 0) \stackrel{(b,(0,1))}{\longrightarrow}(z, 1) \stackrel{\left(c, 1_{1}\right)}{\longrightarrow}(t, 1), \\
& (x, 0) \stackrel{\left.\left(a, 1_{0}\right)\right)}{\longrightarrow}(y, 0) \stackrel{\left.\left(b, 1_{0}\right)\right)}{\longrightarrow}(z, 0) \stackrel{(c,(0,1))}{\longrightarrow}(t, 1),
\end{aligned}
$$

of composable 1 -cells of $\mathcal{T} \times[1]$ are canonically provided by the 3 -cells (38), (39) and (40):

$((G c \otimes G b) \otimes G a) \otimes \theta x \stackrel{\left(\chi^{G} \otimes 1\right) \otimes 1}{=}(G(c \otimes b) \otimes G a) \otimes \theta x$

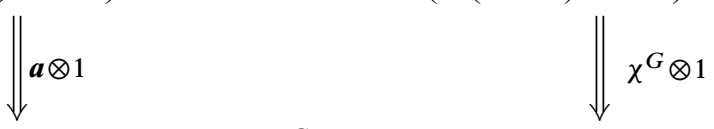

$(G c \otimes(G b \otimes G a)) \otimes \theta x \quad \stackrel{\omega^{G} \otimes 1}{\models} \quad G((c \otimes b) \otimes a) \otimes \theta x$

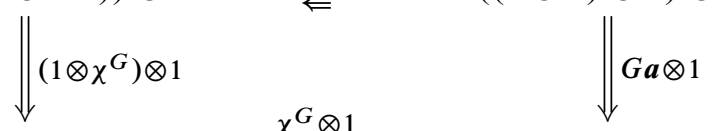

$\left(G c \otimes(G(b \otimes a)) \otimes \theta x \stackrel{\chi^{G} \otimes 1}{=} G(c \otimes(b \otimes a)) \otimes \theta x\right.$

(39)

$(G c \otimes G b) \otimes(\theta y \otimes F a) \stackrel{\chi^{G} \otimes 1}{\Longrightarrow} G(c \otimes b) \otimes(\theta y \otimes F a)$

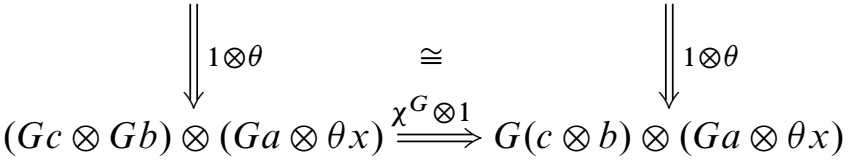

$G c \otimes((\theta z \otimes F b) \otimes F a) \stackrel{1 \otimes(\theta \otimes 1)}{=} G c \otimes((G b \otimes \theta y) \otimes F a)$

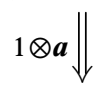

$G c \otimes(\theta z \otimes(F b \otimes F a))$

(40)

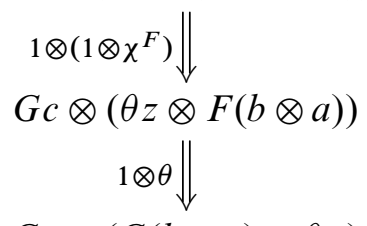

$G c \otimes(G(b \otimes c) \otimes \theta x) \stackrel{1 \otimes\left(\chi^{G} \otimes 1\right)}{\Longleftarrow} G c \otimes((G b \otimes G a) \otimes \theta x)$

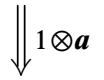

$G c \otimes(G b \otimes(\theta y \otimes F a))$

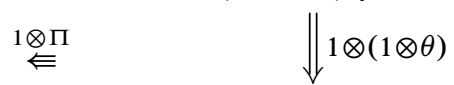

$G c \otimes(G b \otimes(G a \otimes \theta x))$

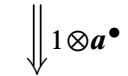

To finish the description of the homomorphism $H$, say that the component of the invertible modification $\delta^{H}$ at any morphism $(a,(0,1)):(x, 0) \rightarrow(y, 1)$ is canonically obtained from the $3-$ cells $1 \otimes \mathrm{M}$ and $\delta^{G} \otimes 1$ below, while the component of $\gamma^{H}$ is 
provided by $3-$ cell $\gamma^{G} \otimes 1$ :
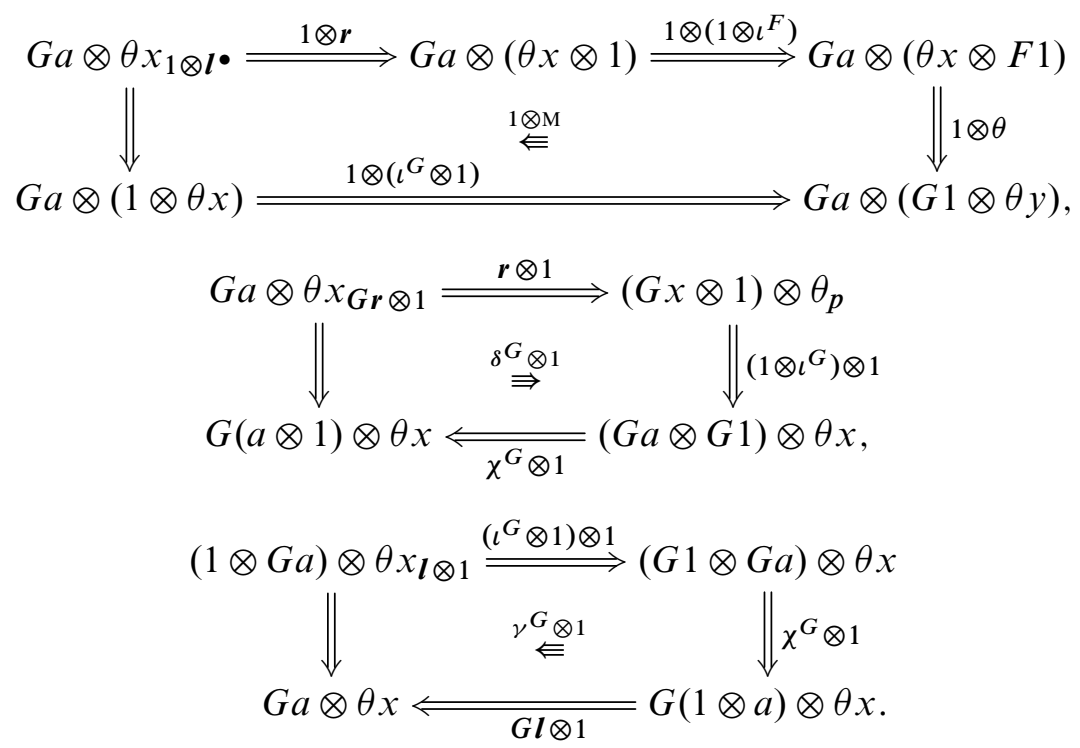

This completes the proof.

Proposition 5.6 If $F, G: \mathcal{T} \rightarrow \mathcal{T}^{\prime}$ are two trihomomorphisms between tricategories, then any tritransformation $F \Rightarrow G$ defines a homotopy between the induced maps on classifying spaces:

$$
\mathrm{B}_{3} F \simeq \mathrm{B}_{3} G: \mathrm{B}_{3} \mathcal{T} \rightarrow \mathrm{B}_{3} \mathcal{T}^{\prime}
$$

Proof Taking geometric nerves in the diagram (37), we obtain a commutative diagram of simplicial maps

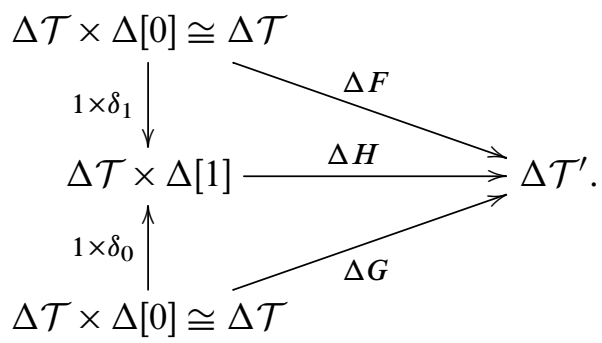

Since $|\Delta[1]| \cong[0,1]$, the unity interval, the result follows by Theorem 5.4 .

As a consequence for triequivalences between tricategories, we have the following. 
Corollary 5.7 (i) If $F: \mathcal{T} \rightarrow \mathcal{T}^{\prime}$ is any trihomomorphism such that there is a trihomomorphism $G: \mathcal{T}^{\prime} \rightarrow \mathcal{T}$ and tritransformations $F G \Rightarrow 1_{\mathcal{T}^{\prime}}$ and $1_{\mathcal{T}} \Rightarrow G F$, then the induced map $\mathrm{B}_{3} F: \mathrm{B}_{3} \mathcal{T} \rightarrow \mathrm{B}_{3} \mathcal{T}^{\prime}$ is a homotopy equivalence.

(ii) Any triequivalence of tricategories induces a homotopy equivalence on classifying spaces.

Proof (i) Given a trihomomorphism $F: \mathcal{T} \rightarrow \mathcal{T}^{\prime}$ as in the hypothesis, there is a homotopy $\mathrm{B}_{3} F \mathrm{~B}_{3} G \simeq \mathrm{B}_{3}(F G)$ by Proposition 4.10(ii). By Proposition 5.6, the existence of a homotopy $\mathrm{B}_{3}(F G) \simeq \mathrm{B}_{3} 1_{\mathcal{T}}$, follows. Since, by Proposition 4.10 (iii), there is a homotopy $\mathrm{B}_{3} 1_{\mathcal{T}^{\prime}} \simeq 1_{\mathrm{B}_{3} \mathcal{T}^{\prime}}$, we conclude the existence of a homotopy $\mathrm{B}_{3} F \mathrm{~B}_{3} G \simeq 1_{\mathrm{B}_{3} \mathcal{T}^{\prime}}$. Analogously, we can prove that $1_{\mathrm{B}_{3} \mathcal{T}} \simeq \mathrm{B}_{3} G \mathrm{~B}_{3} F$, which completes the proof of this part. Part (ii) clearly follows from part (i).

Example 5.8 (Geometric nerves of 3-categories) In [43], Street gave a precise notion of nerve for $n$-categories. He extended each graph $\mathcal{G}_{p}=(0 \rightarrow 1 \rightarrow \cdots \rightarrow p)$ to a "free" $\omega$-category $\mathcal{O}_{p}$ (called the $p^{\text {th }}$-oriental) such that for any $n$-category $\mathcal{X}$, the $p$-simplices of its nerve are just $n$-functors $\mathcal{O}_{p} \rightarrow \mathcal{X}$ from the underlying $n$-category of the $p^{\text {th }}$-oriental to $\mathcal{X}$. In the case when $n=3$, Street's nerve construction on a 3 -category $\mathcal{T}$ just produces, up to some directional changes, its geometric nerve $\Delta \mathcal{T}$ as stated in Definition 5.1. After the discussion in Example 4.11, from Theorem 5.4 we get homotopy equivalences

$$
|\operatorname{diag} \mathrm{NNN} \mathcal{T}| \simeq \mathrm{B}_{3} T \simeq|\Delta \mathcal{T}|
$$

for any 3-category $\mathcal{T}$.

Example 5.9 (Geometric nerves of braided monoidal categories) If $A$ is any abelian group, then the braided monoidal category with only one object it defines, $(A,+, 0)$, has as double suspension the tricategory $\Sigma^{2} A$ treated in Examples 2.1 and 2.2. For any integer $p \geq 0$, we have

$$
\operatorname{Lax}_{\mathrm{u}}\left([p], \Sigma^{2} A\right)=Z^{3}([p], A)=Z^{3}(\Delta[p], A)=K(A, 3)_{p},
$$

whence $\Delta \Sigma^{2} A=K(A, 3)$, the minimal Eilenberg-Mac Lane complex. Hence, from Theorem 5.4 and Corollary 4.19, it follows that $\mathrm{B}_{3}(A,+, 0)=|K(A, 3)|$.

If $(C, \otimes, c)$ is any braided monoidal category, then a unitary lax functor of a category $I$ in the double suspension tricategory, $I \rightarrow \Sigma^{2}(C, \otimes, c)$, is what was called a (normal) 3-cocycle of $I$ with coefficients in the braided monoidal category by Carrasco, Cegarra and Garzón in [12, Definition 6.6] and by Cegarra and Khmaladze 
in $\left[14\right.$, Section 4]. Therefore, the geometric nerve $\Delta \Sigma^{2}(C, \otimes, c)$ coincides with the simplicial set [12, Definition 6.7]

$$
Z^{3}(C, \otimes, c): \Delta^{\mathrm{op}} \rightarrow \text { Set }, \quad[p] \mapsto Z^{3}([p],(C, \otimes, c))
$$

whose $p$-simplices are the 3-cocycles of $[p]$ in the braided monoidal category. The geometric nerve $Z^{3}(C, \otimes, c)$ is then a 4 -coskeletal 1 -reduced (one vertex, one 1simplex) simplicial set, whose 2-simplices are the objects $F_{0,1,2}$ of $C$, and whose $p$-simplices, for $p \geq 3$, are families of morphisms of the form

$$
F_{i, j, k, l}: F_{i, j, l} \otimes F_{j, k, l} \rightarrow F_{i, k, l} \otimes F_{i, j, k}, \quad 0 \leq i<j<k<l \leq p
$$

that make the diagram

$$
\begin{array}{cc}
F_{i, j, m} \otimes\left(F_{j, k, m} \otimes F_{k, l, m}\right) & \boldsymbol{a}\left(F_{i, j, k, m} \otimes 1\right) \boldsymbol{a}^{-1} \\
1 \otimes F_{j, k, l, m} \downarrow & F_{i, k, m} \otimes\left(F_{i, j, k} \otimes F_{k, l, m}\right) \\
F_{i, j, m} \otimes\left(F_{j, l, m} \otimes F_{j, k, l}\right) & \mid 1 \otimes \boldsymbol{c} \\
\left(F_{i, j, l, m} \otimes 1\right) \boldsymbol{a}^{-1} \downarrow & F_{i, k, m} \otimes\left(F_{k, l, m} \otimes F_{i, j, k}\right) \\
\left(F_{i, l, m} \otimes F_{i, j, l}\right) \otimes F_{j, k, l} \stackrel{\boldsymbol{a}^{-1}\left(1 \otimes F_{i, j, k, l}\right) \boldsymbol{a}}{\longrightarrow} & \downarrow\left(F_{i, l, m} \otimes F_{i, k, l, m} \otimes 1\right) \boldsymbol{a}^{-1} \\
&
\end{array}
$$

commutative for $0 \leq i<j<k<l<m \leq p$. From Theorem 5.4 and Corollary 4.19, we obtain the following known result.

Corollary 5.10 [12, Theorem 6.11] For any braided monoidal category $(C, \otimes, c)$, there is a homotopy equivalence $\mathrm{B}_{3}(C, \otimes, c) \simeq\left|Z^{3}(C, \otimes, c)\right|$.

Example 5.11 (Geometric nerves of monoidal bicategories) If $(\mathcal{B}, \otimes)$ is any monoidal bicategory, then we define its geometric nerve, denoted by $\Delta(\mathcal{B}, \otimes)$, as the geometric nerve of its suspension 3-category $\Sigma(\mathcal{B}, \otimes)$ from (23). That is,

$$
\Delta(\mathcal{B}, \otimes): \Delta^{\mathrm{op}} \rightarrow \text { Simpl.Set }, \quad[p] \mapsto \operatorname{Lax}_{\mathrm{u}}([p], \Sigma(\mathcal{B}, \otimes)) .
$$

Then Theorem 5.4 specializes to monoidal bicategories, giving a homotopy equivalence

$$
\mathrm{B}_{3}(\mathcal{B}, \otimes) \simeq|\Delta(\mathcal{B}, \otimes)| .
$$

\subsection{Bicategorical groups and homotopy 3-types}

Recall that a bigroupoid is a bicategory $\mathcal{B}$ in which every 2 -cell is invertible. This means that all the hom categories $\mathcal{B}(x, y)$ are groupoids, and every 1-cell $u: x \rightarrow y$ is an equivalence, that is, there exist a morphism $u^{\prime}: y \rightarrow x$ and 2-cells $u \circ u^{\prime} \Rightarrow 1_{y}$ 
and $1_{x} \Rightarrow u^{\prime} \circ u$. By a bicategorical group we shall mean a monoidal bigroupoid $(\mathcal{B}, \otimes)$ in which for every object $x$ there is an object $x^{\prime}$ with 1 -cells $1 \rightarrow x \otimes x^{\prime}$ and $x^{\prime} \otimes x \rightarrow 1$. Bicategorical groups correspond to those Picard 2-categories, in the sense of Gurski [24, Section 6], whose underlying bicategory is a bigroupoid.

In any bicategorical group $(\mathcal{B}, \otimes)$, the homomorphisms $x \otimes-: \mathcal{B} \rightarrow \mathcal{B}$ and $-\otimes x: \mathcal{B} \rightarrow \mathcal{B}$ are biequivalences for any object $x \in \mathcal{B}$. Hence, by Theorem 4.17 , there is a homotopy equivalence

$$
\mathrm{B}_{2} \mathcal{B} \simeq \Omega \mathrm{B}_{3}(\mathcal{B}, \otimes)
$$

between the classifying space of the underlying bigroupoid and the loop space of the classifying space of the bicategorical group.

If $(\mathcal{B}, \otimes)$ is any monoidal bicategory, then its geometric nerve $\Delta(\mathcal{B}, \otimes)$ is a 4 coskeletal reduced (one vertex) simplicial set, which satisfies the Kan extension condition if and only if $(\mathcal{B}, \otimes)$ is a bicategorical group. In such a case, the homotopy groups of its geometric realization

$$
\pi_{i} \mathrm{~B}_{3}(\mathcal{B}, \otimes) \cong \pi_{i} \Delta(\mathcal{B}, \otimes) \cong \pi_{i-1} \Delta \mathcal{B}
$$

are plainly recognized to be:

- $\pi_{i} \mathrm{~B}_{3}(\mathcal{B}, \otimes)=0$ if $i \neq 1,2,3$

- $\pi_{1} \mathrm{~B}_{3}(\mathcal{B}, \otimes)=\mathrm{Ob} \mathcal{B} / \sim$, the group of equivalence classes of objects in $\mathcal{B}$ where multiplication is induced by the tensor product

- $\pi_{2} \mathrm{~B}_{3}(\mathcal{B}, \otimes)=\operatorname{Aut}_{\mathcal{B}}(1) / \cong$, the group of isomorphism classes of autoequivalences of the unit object where the operation is induced by the horizontal composition in $\mathcal{B}$

- $\pi_{3} B_{3}(\mathcal{B}, \otimes)=\operatorname{Aut}_{\mathcal{B}}\left(1_{1}\right)$, the group of automorphisms of the identity 1 -cell of the unit object where the operation is vertical composition in $\mathcal{B}$

Thus bicategorical groups arise as algebraic path-connected homotopy 3-types, a fact that supports the Homotopy hypothesis of Baez [1]. Indeed, every path-connected homotopy 3-type can be realized in this way from a bicategorical group, as we show below (see Berger [5], Joyal and Tierney [30], Lack [33] or Leroy [35] for alternative approaches to this issue).

Proposition 5.12 For any path-connected pointed $C W$-complex $X$ with $\pi_{i} X=0$ for $i \geq 4$, there is a bicategorical group $(\mathcal{B}(X), \otimes)$ whose classifying space $\mathrm{B}_{3}(\mathcal{B}(X), \otimes)$ is homotopy equivalent to $X$. 
Proof Given $X$ as above, let $M(X) \subseteq S(X)$ be a minimal subcomplex that is a deformation retract of the total singular complex of $X$, so that $|M(X)| \simeq X$. Taking into account the Postnikov $k$-invariants, this minimal complex $M$ can be described (see Goerss and Jardine [19, VI, Corollary 5.13]) up to isomorphism as

$$
M(X)=K(B, 3) \times_{t}\left(K(A, 2) \times_{h} K(G, 1)\right)
$$

by means of the group $G=\pi_{1} X$, the $G$-modules $A=\pi_{2} X$ and $B=\pi_{3} X$, and two maps

$$
h: G^{3} \rightarrow A, \quad t: A^{6} \times G^{4} \rightarrow B
$$

defining normalized cocycles $h \in Z^{3}(G, A)$ and $t \in Z^{4}\left(K(A, 2) \times_{h} K(G, 1), B\right)$. That is, $M(X)$ is the $4^{\text {th }}$ coskeleton of the truncated simplicial set

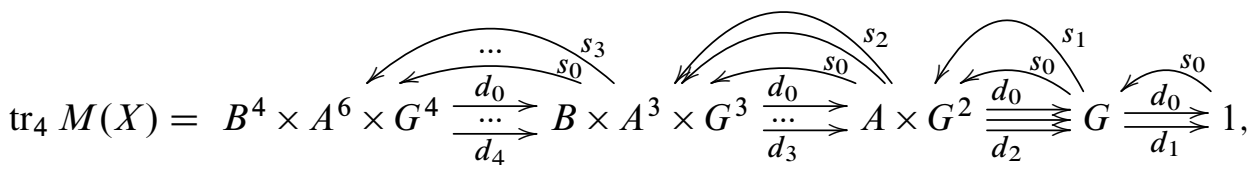

whose face and degeneracy operators are given by $\left(\sigma_{i} \in G, x_{j} \in A, u_{k} \in B\right)$ :

$d_{i}\left(x_{1}, \sigma_{1}, \sigma_{2}\right)= \begin{cases}\sigma_{2} & i=0, \\ \sigma_{1} \sigma_{2} & i=1 \\ \sigma_{1} & i=2\end{cases}$

$d_{i}\left(u_{1}, x_{1}, x_{2}, x_{3}, \sigma_{1}, \sigma_{2}, \sigma_{3}\right)= \begin{cases}\left(\sigma_{1}^{-1} x_{3}, \sigma_{2}, \sigma_{3}\right) & i=0 \\ \left(x_{2}+x_{3}, \sigma_{1} \sigma_{2}, \sigma_{3}\right) & i=1 \\ \left(x_{1}+x_{2}, \sigma_{1}, \sigma_{2} \sigma_{3}\right) & i=2 \\ \left(x_{1}-h\left(\sigma_{1}, \sigma_{2}, \sigma_{3}\right), \sigma_{1}, \sigma_{2}\right) & i=3\end{cases}$

$d_{i}\left(u_{1}, u_{2}, u_{3}, u_{4}, x_{1}, x_{2}, x_{3}, x_{4}, x_{5}, x_{6}, \sigma_{1}, \sigma_{2}, \sigma_{3}, \sigma_{4}\right)$

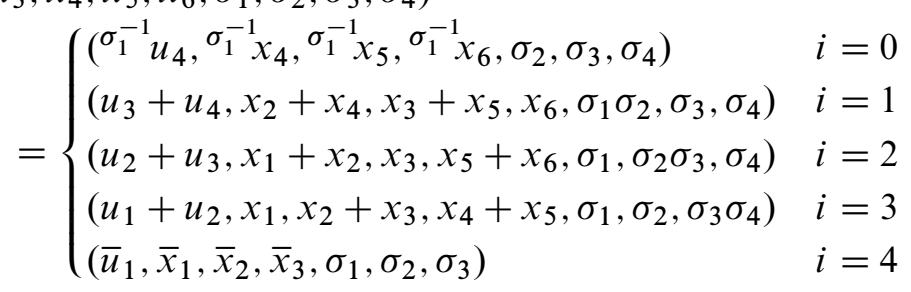

where we have that

$$
\begin{aligned}
& \bar{u}_{1}=u_{1}-t\left(x_{1}, x_{2}, x_{3}, x_{4}, x_{5}, x_{6}, \sigma_{1}, \sigma_{2}, \sigma_{3}, \sigma_{4}\right), \\
& \bar{x}_{1}=x_{1}-h\left(\sigma_{1}, \sigma_{2}, \sigma_{3} \sigma_{4}\right)+h\left(\sigma_{1}, \sigma_{2}, \sigma_{3}\right), \\
& \bar{x}_{2}=x_{2}-h\left(\sigma_{1} \sigma_{2}, \sigma_{3}, \sigma_{4}\right)+{ }_{1}^{-1} h\left(\sigma_{2}, \sigma_{3}, \sigma_{4}\right) \\
& \bar{x}_{3}=x_{4}-\sigma_{1}^{-1} h\left(\sigma_{2}, \sigma_{3}, \sigma_{4}\right) .
\end{aligned}
$$


Then we can define a bicategorical group $(\mathcal{B}(X), \otimes)$ with a simplicial isomorphism $\Delta(\mathcal{B}(X), \otimes) \cong M(X)$ as follows.

- A 0 -cell of $\mathcal{B}=\mathcal{B}(X)$ is an element $\sigma \in G$. If $\sigma \neq \tau$ are different elements of $G$ then $\mathcal{B}(\sigma, \tau)=\varnothing$, that is, there is no 1 -cell between them, whereas if $\sigma=\tau$ then the $1-$ cell $x: \sigma \rightarrow \sigma$ is an element $x \in A$. Similarly, there is no any $2-$ cell in $\mathcal{B}$ between two 1-cells $x, y: \sigma \rightarrow \sigma$ if $x \neq y$, whereas when $x=y$, the 2-cell $u: x \Rightarrow x$ is an element $u \in B$.

- The vertical composition of 2 -cells is given by addition in $B$, that is,

$$
(x \stackrel{u}{\Longrightarrow} x) \cdot(x \stackrel{v}{\Longrightarrow} x)=(x \stackrel{u+v}{\Longrightarrow} x) .
$$

- The horizontal composition of 1-cells and 2-cells is given by addition in $A$ and $B$ respectively, that is,

$$
(\sigma \overbrace{x}^{\frac{\Downarrow u}{\Downarrow}} \sigma) \circ(\sigma \overbrace{\frac{\Downarrow v}{y}}^{y} \sigma)=(\sigma \overbrace{x+y}^{\frac{x+y}{\Downarrow u+v} \sigma}) .
$$

- The associativity isomorphism is

$$
\sigma \overbrace{x+(y+z)}^{(x+y)+z} \sigma, \quad \boldsymbol{a}=t(x, y, z, 0,0,0, \sigma, 1,1,1)
$$

and the 0 of $A$ gives the (strict) unit on each $\sigma$, that is, $1_{\sigma}=0: \sigma \rightarrow \sigma$.

- The (strictly unitary) tensor homomorphism $\otimes: \mathcal{B} \times \mathcal{B} \rightarrow \mathcal{B}$ is given on cells of $\mathcal{B}$ by

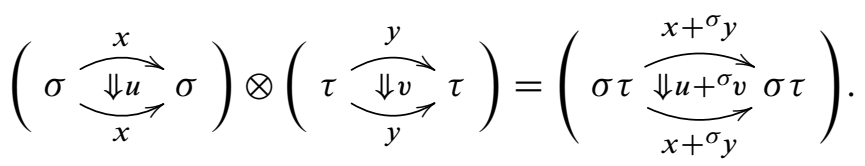

- The structure interchange isomorphism

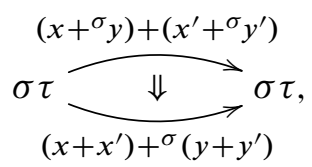

for any $1-$ cells $\sigma \stackrel{x^{\prime}}{\rightarrow} \sigma \stackrel{x}{\rightarrow} \sigma$ and $\tau \stackrel{y^{\prime}}{\rightarrow} \tau \stackrel{y}{\rightarrow} \tau$ is the one obtained by pasting into the bigroupoid $\mathcal{B}$ the diagram 


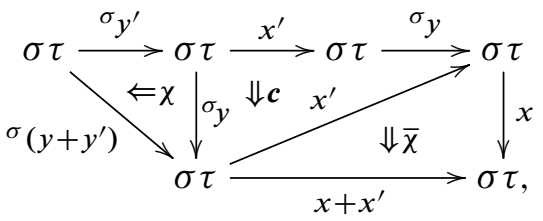

where

$$
\begin{aligned}
\chi=-t\left(0,0,0,{ }^{\sigma} y,{ }^{\sigma} y^{\prime}, 0, \sigma, \tau, 1,1\right) & \\
c=t\left(0, x, 0,0,{ }^{\sigma} y, 0, \sigma, \tau, 1,1\right)-t\left(0,0, x,{ }^{\sigma} y, 0,0, \sigma, \tau, 1,1\right) & \\
& -t\left(x, 0,0,0,0,{ }^{\sigma} y, \sigma, 1, \tau, 1\right), \\
\bar{\chi}=-t\left(0, x, x^{\prime}, 0,0,0, \sigma, \tau, 1,1\right)+t\left(x, 0, x^{\prime}, 0,0,0, \sigma, 1, \tau, 1\right) & -t\left(x, x^{\prime}, 0,0,0,0, \sigma, 1,1, \tau\right) .
\end{aligned}
$$

- The associativity pseudoequivalence $(-\otimes-) \otimes-\stackrel{a}{\Rightarrow}-\otimes(-\otimes-): \mathcal{B}^{3} \rightarrow \mathcal{B}$ is defined by the 1 -cells

$$
h(\sigma, \tau, \gamma):(\sigma \tau) \gamma \rightarrow \sigma(\tau \gamma)
$$

The naturality component of $\boldsymbol{a}$, at any $1-$ cells $\sigma \stackrel{x}{\rightarrow} \sigma, \tau \stackrel{y}{\rightarrow} \tau$ and $\gamma \stackrel{z}{\rightarrow} \gamma$,

$$
\begin{aligned}
(\sigma \tau) \gamma & \stackrel{h=h(\sigma, \tau, \gamma)}{\longrightarrow} \sigma(\tau \gamma) \\
\left(x+{ }^{\sigma} y\right)+{ }^{\sigma \tau} z & \Rightarrow \\
(\sigma \tau) \gamma \stackrel{{ }^{\prime}}{h(\sigma, \tau, \gamma)} & \Rightarrow(\tau \gamma)
\end{aligned}
$$

is given by pasting in $\mathcal{B}$ the diagram

where

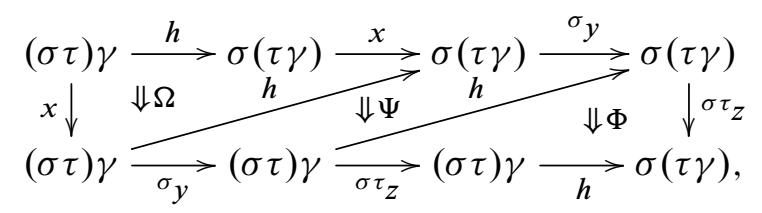

$$
\begin{aligned}
& \Phi=t\left(0, h, 0,0,{ }^{\sigma \tau} z, 0, \sigma, \tau \gamma, 1,1\right)-t\left(h, 0,0,0,0,{ }^{\sigma \tau} z, \sigma, \tau, \gamma, 1\right) \\
& -t\left(0,0, h{ }^{\sigma \tau} z, 0,0, \sigma, \tau \gamma, 1,1\right), \\
& \Psi=t\left(h, 0,0,{ }^{\sigma} y, 0,0, \sigma, \tau, 1, \gamma\right)-t\left(h, 0,0,0,{ }^{\sigma} y, 0, \sigma, \tau, \gamma, 1\right) \\
& +t\left(0, h, 0,0,{ }^{\sigma} y, 0, \sigma, \tau \gamma, 1,1\right)-t\left(0,0, h,{ }^{\sigma} y, 0,0, \sigma, \tau \gamma, 1,1\right), \\
& \Omega=-t(x, h, 0,0,0,0, \sigma, 1, \tau, \gamma)+t(h, x, 0,0,0,0, \sigma, \tau, 1, \gamma) \\
& -t(h, 0, x, 0,0,0, \sigma, \tau, \gamma, 1)+t(x, 0, h, 0,0,0, \sigma, 1, \tau \gamma, 1) \\
& +t(0, h, x, 0,0,0, \sigma, \tau \gamma, 1,1)-t(0, x, h, 0,0,0, \sigma, \tau \gamma, 1,1) .
\end{aligned}
$$


- The structure modification $\pi$ at any objects $\sigma, \tau, \gamma, \delta \in G$ is

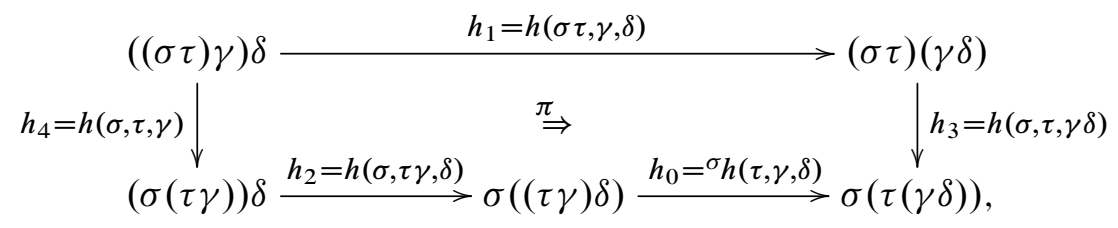

where

$$
\begin{aligned}
\pi=t & \left(h_{3}, h_{1}-h_{0}, 0, h_{0}, 0,0, \sigma, \tau, \gamma, \delta\right) \\
& -t\left(h_{2}, h_{4}, 0,0,0,0, \sigma, \tau \gamma, 1, \delta\right)+t\left(h_{2}, 0, h_{4}, 0,0,0, \sigma, \tau \gamma, \delta, 1\right) \\
& -t\left(h_{3}, 0, h_{1}-h_{0}, 0, h_{0}, 0, \sigma, \tau, \gamma \delta, 1\right)+t\left(0, h_{3}, h_{1}-h_{0}, 0, h_{0}, 0, \sigma, \tau \gamma \delta, 1,1\right) \\
& -t\left(0,0, h_{2}+h_{4}, h_{0}, 0,0, \sigma, \tau \gamma \delta, 1,1\right)-t\left(0, h_{2}, h_{4}, 0,0,0, \sigma, \tau \gamma \delta, 1,1\right) .
\end{aligned}
$$

This completes the description of bicategorical group $(\mathcal{B}, \otimes)=(\mathcal{B}(X), \otimes)$, whose geometric nerve is recognized to be isomorphic to the minimal complex $M(X)$ in (41) by means of the simplicial map $\varphi: \Delta(\mathcal{B}, \otimes) \rightarrow M$ which, in dimensions less than or equal to 4 fits into the diagram

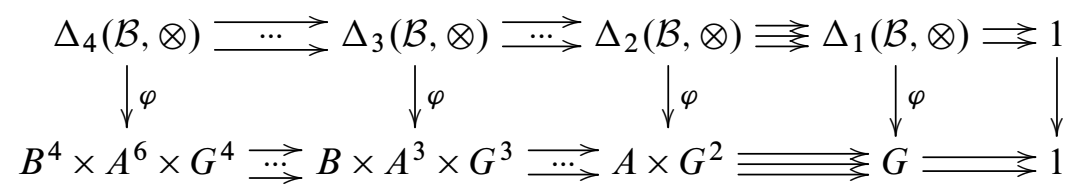

and carries (keeping the notation in (25)-(29)):

- a unitary lax functor $F:[1] \rightarrow \Sigma(\mathcal{B}, \otimes)$ to $\varphi(F)=F_{0,1}$

- a unitary lax functor $F:[2] \rightarrow \Sigma(\mathcal{B}, \otimes)$ to $\varphi(F)=\left(-F_{0,1,2}, F_{0,1}, F_{1,2}\right)$

- a unitary lax functor $F:[3] \rightarrow \Sigma(\mathcal{B}, \otimes)$ to

$$
\begin{aligned}
\varphi(F)=\left(-F_{0,1,2,3},-{ }^{F_{0,1}} F_{1,2,3}+F_{0,2,3}-F_{0,1,3},\right. & F_{0,1} F_{1,2,3}-F_{0,2,3}, \\
& \left.-{ }^{F_{0,1}} F_{1,2,3}, F_{0,1}, F_{1,2}, F_{2,3}\right)
\end{aligned}
$$

- a $F:[4] \rightarrow \Sigma(\mathcal{B}, \otimes)$ to

$$
\varphi(F)=\left(u_{1}, u_{2}, u_{3}, u_{4}, x_{1}, x_{2}, x_{3}, x_{4}, x_{5}, x_{6}, F_{0,1}, F_{1,2}, F_{2,3}, F_{3,4}\right),
$$

where:

$$
\begin{aligned}
& u_{1}={ }^{F_{0,1}} F_{1,2,3,4}-F_{0,1,2,4}+F_{0,1,3,4}-F_{0,2,3,4} \\
& u_{2}=F_{0,2,3,4}-F_{0,1,3,4}-{ }^{F_{0,1}} F_{1,2,3,4} \\
& u_{3}={ }^{F_{0,1}} F_{1,2,3,4}-F_{0,2,3,4}
\end{aligned}
$$




$$
\begin{aligned}
& u_{4}=-{ }^{F_{0,1}} F_{1,2,3,4} \\
& x_{1}=F_{0,2,4}-{ }^{F_{0,1}} F_{1,2,4}-F_{0,1,4} \\
& x_{2}={ }^{F_{0,1}} F_{1,2,4}-{ }^{F_{0,1}} F_{1,3,4}+F_{0,3,4}-F_{0,2,4} \\
& x_{3}={ }^{F_{0,1}} F_{1,3,4}-F_{0,3,4} \\
& x_{4}={ }^{F_{0,1}} F_{1,3,4}-{ }^{F_{0,1}} F_{1,2,4}-{ }^{F_{0,2}} F_{2,3,4} \\
& x_{5}={ }^{F_{0,2}} F_{2,3,4}-{ }^{F_{0,1}} F_{1,3,4} \\
& x_{6}=-{ }^{F_{0,2}} F_{2,3,4}
\end{aligned}
$$

This completes the proof.

Remark 5.13 The fundamental bigroupoid $\Pi_{2}(X)$ of a space $X$ was independently described by Hardie, Kamps and Kieboom in [26] and by Stevenson in [42]. The objects of $\Pi_{2}(X)$ are the points $x \in X$, the 1 -cells $f: x \rightarrow y$ are paths $f: I=[0,1] \rightarrow X$ with $f(0)=x$ and $f(1)=y$, and the $2-$ cells $[\alpha]: f \Rightarrow g$ are relative homotopy classes of homotopies between paths $\alpha: I \times I \rightarrow X$ with $\left.\alpha\right|_{I \times 0}=f$ and $\left.\alpha\right|_{I \times 1}=g$. In [23, Theorem 1.4], Gurski proves that when the space $X$ is endowed with a structure of algebra for the little cubes operad $\mathcal{C}_{1}$, then $\Pi_{2}(X)$ has the structure of a monoidal bicategory, say $\left(\Pi_{2}(X), \otimes\right)$. In this way, any given pointed topological space $X$ has associated a bicategorical group $\left(\Pi_{2}(\Omega X), \otimes\right)$, which is Gurski's monoidal bicategory of the loop space $\Omega X$. Although we will not give details here, we want to point out that for $X$ a path-connected pointed space $X$ for which $\pi_{i} X=0$ for $i \geq 4$, the bicategorical group $(\mathcal{B}(X), \otimes)$ in Proposition 5.12 is a skeleton of the bicategorical group $\left(\Pi_{2}(\Omega X), \otimes\right)$. That is, there is a monoidal biequivalence $(\mathcal{B}(X), \otimes) \simeq\left(\Pi_{2}(\Omega X), \otimes\right)$, and the bigroupoid $\mathcal{B}(X)$ is skeletal in the sense that any two isomorphic 1-cells are equal and any two equivalent objects are equal.

To finish, we shall remark on two particular relevant cases of the demonstrated relationship between monoidal bicategories and path-connected homotopy 3-types. Since categorical groups, in the sense of Joyal and Street [29, Section 3], are the same thing as bicategorical groups in which all 2-cells are identities, then categorical groups are algebraic models for path-connected homotopy 2-types; see Carrasco and Cegarra [9, Section 2.1] and Cegarra and Garzón [13, Section 5]. This fact goes back to Whitehead (1949) and Mac Lane and Whitehead (1950) since every categorical group is equivalent to a strict one, and strict categorical groups are the same as crossed modules. On the other hand, if $(C, \otimes, c)$ is any braided categorical group [29], then its classifying space $\mathrm{B}_{3}(C, \otimes, c)$ is the classifying space of its suspension bicategorical group $(\Sigma(C, \otimes), \otimes)$ (see Examples 4.18 and 5.9), which is precisely a one-object bicategorical group. Therefore, we conclude from the above discussion that braided categorical groups are 
algebraic models for path-connected simply connected homotopy 3-types, a fact due to Joyal and Tierney [30], but also proved by Carrasco and Cegarra in [9, Section 2.2] (cf [10, Theorem 3.8]) and, implicitly, by Joyal and Street in [29, Theorem 3.3].

\section{Appendix: Coherence conditions}

(CR1) For any four composable arrows in $I, i \stackrel{d}{\longrightarrow} j \stackrel{c}{\longrightarrow} k \stackrel{b}{\longrightarrow} l \stackrel{a}{\longrightarrow} m$, the equation $A=A^{\prime}$ on 3 -cells in $\mathcal{T}$ holds, where $A$ is given by:

$((F a \otimes F b) \otimes F c) \otimes F d \stackrel{a}{\Longrightarrow}(F a \otimes F b) \otimes(F c \otimes F d) \stackrel{a}{\Longrightarrow} F a \otimes(F b \otimes(F c \otimes F d))$

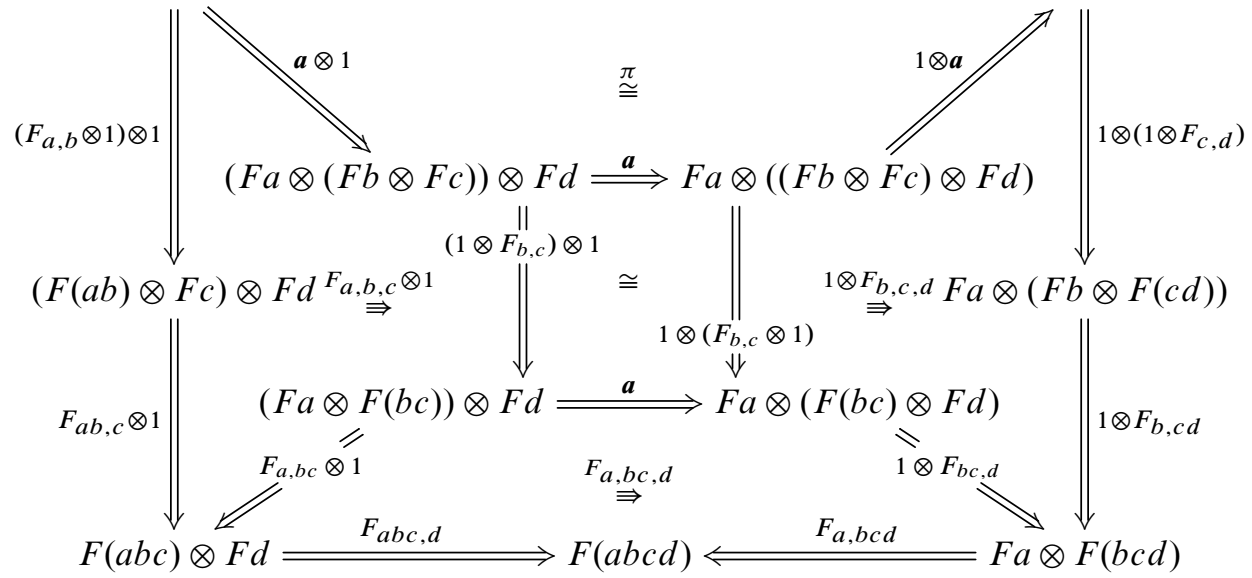

$A^{\prime}$ is given by:

$((F a \otimes F b) \otimes F c) \otimes F d \stackrel{a}{\Longrightarrow}(F a \otimes F b) \otimes(F c \otimes F d) \stackrel{a}{\Longrightarrow} F a \otimes(F b \otimes(F c \otimes F d))$
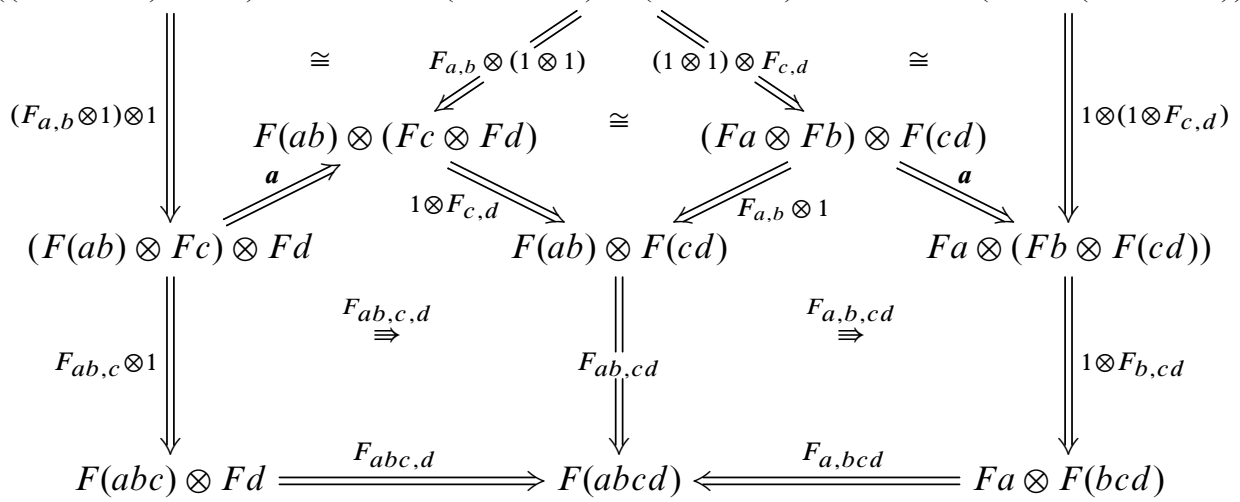

(CR2) For any two composable arrows $i \stackrel{b}{\longrightarrow} j \stackrel{a}{\longrightarrow} k$ in $I$, the equations $B=B^{\prime}$, $C=C^{\prime}$ and $D=D^{\prime}$, on 3 -cells in $\mathcal{T}$ hold, where $B$ is given by 


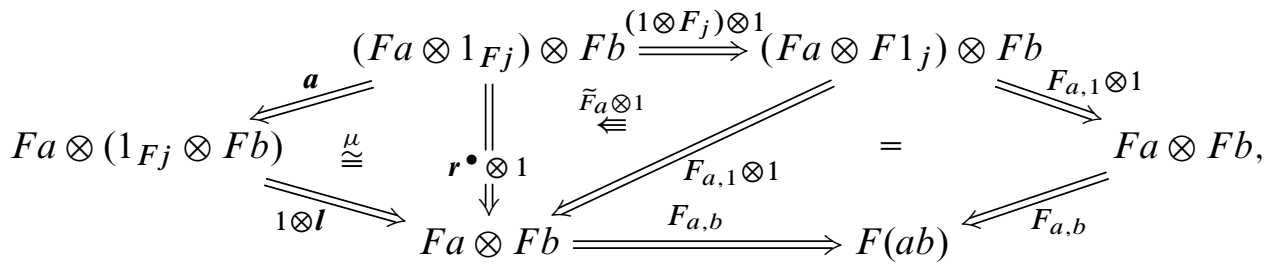

$B^{\prime}$ is given by:

$$
\left(F a \otimes 1_{F j}\right) \otimes F b \stackrel{\left(1 \otimes F_{j}\right) \otimes 1}{=}\left(F a \otimes F 1_{j}\right) \otimes F b
$$

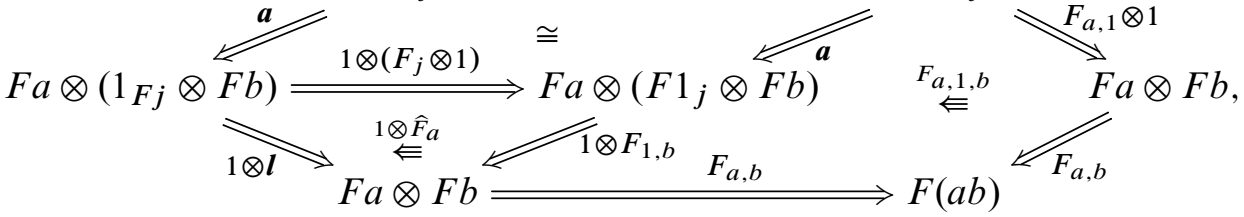

$C$ is given by:

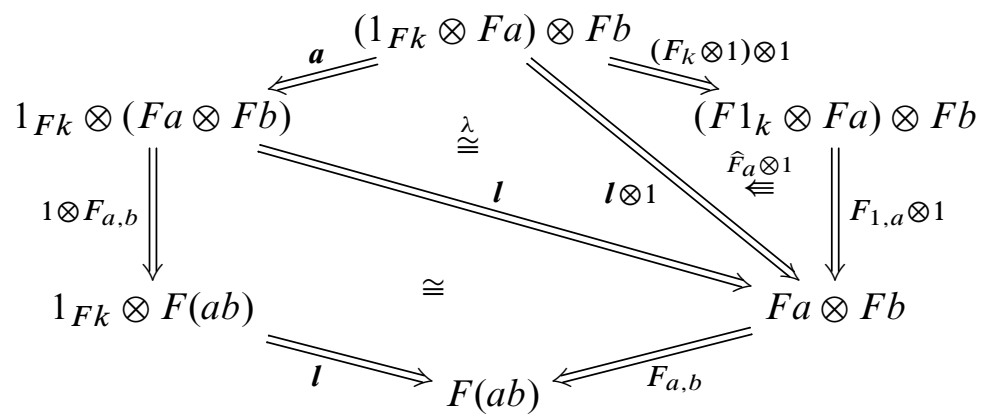

$C^{\prime}$ is given by:

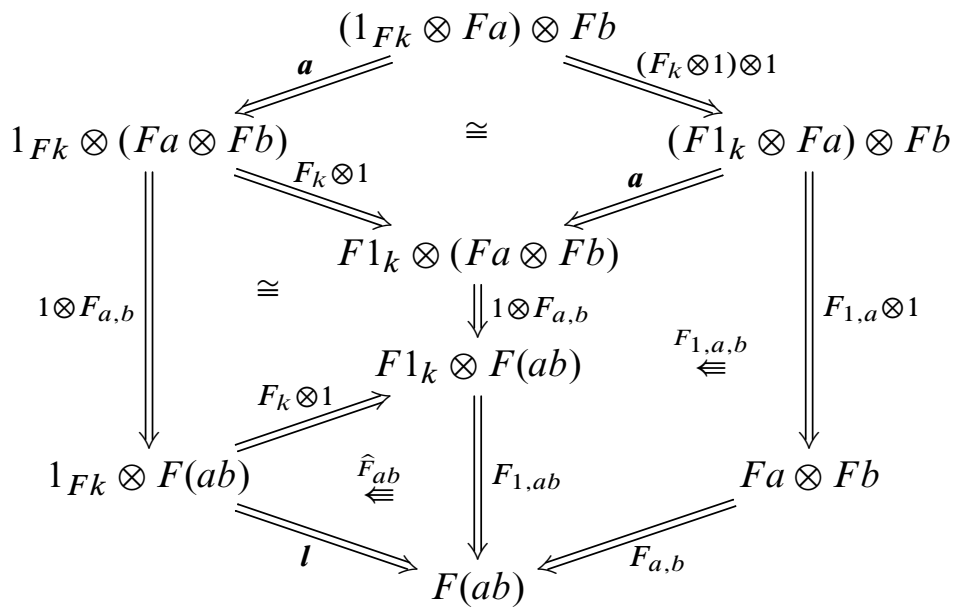


$D$ is given by:

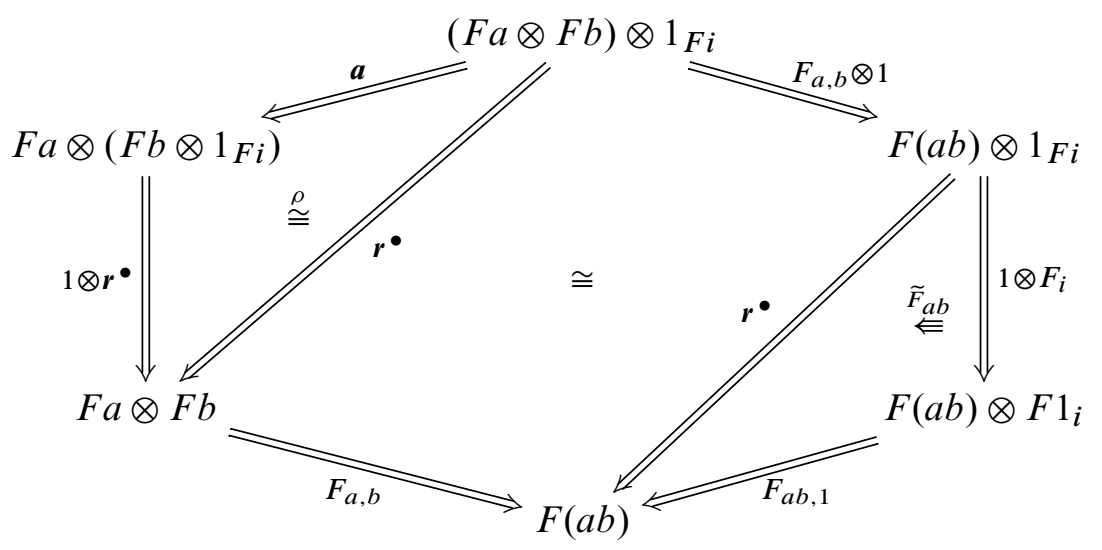

$D^{\prime}$ is given by:

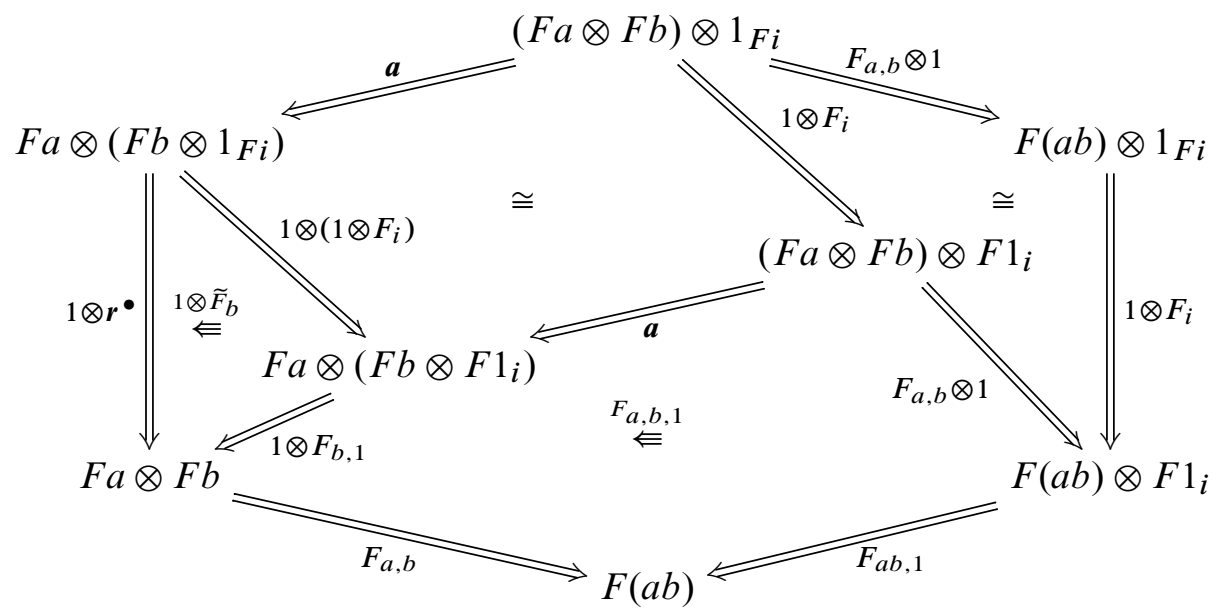

(CR3) For any object $i \in I$,the following equation holds:

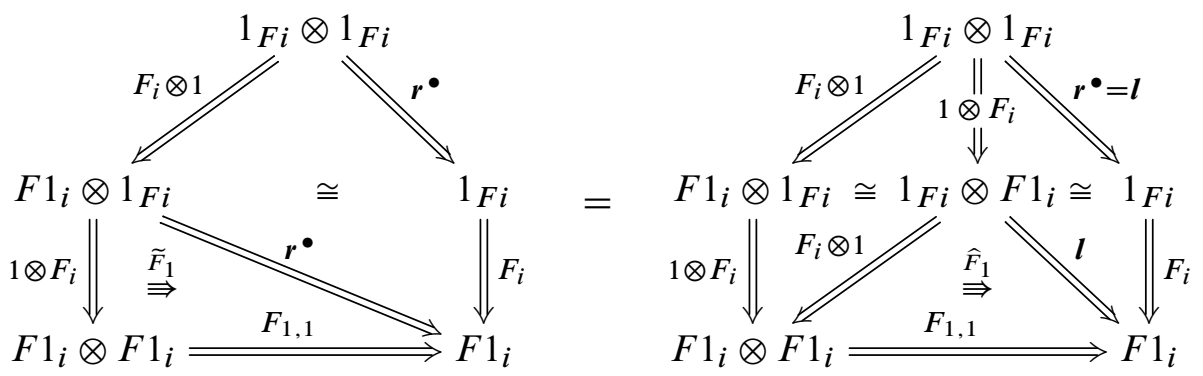


(CR4) For any triplet of composable morphisms of $I, i \stackrel{c}{\longrightarrow} j \stackrel{b}{\longrightarrow} k \stackrel{a}{\longrightarrow} l$, the equation $E=E^{\prime}$ on 3 -cells in $\mathcal{T}$ holds, where $E$ is given by:

$(F a \otimes F b) \otimes F c \stackrel{a}{\Longrightarrow} F a \otimes(F b \otimes F c) \stackrel{1 \otimes F_{b, c}}{=} F a \otimes F(b c)$

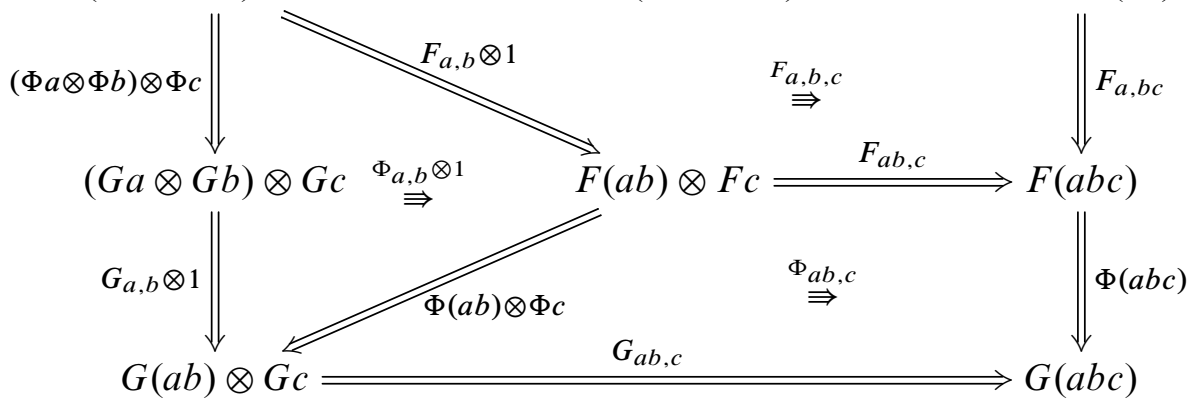

$E^{\prime}$ is given by:

$(F a \otimes F b) \otimes F c=\frac{a}{\Longrightarrow} F a \otimes(F b \otimes F c)=\frac{1 \otimes F_{b, c}}{=} F a \otimes F(b c)$

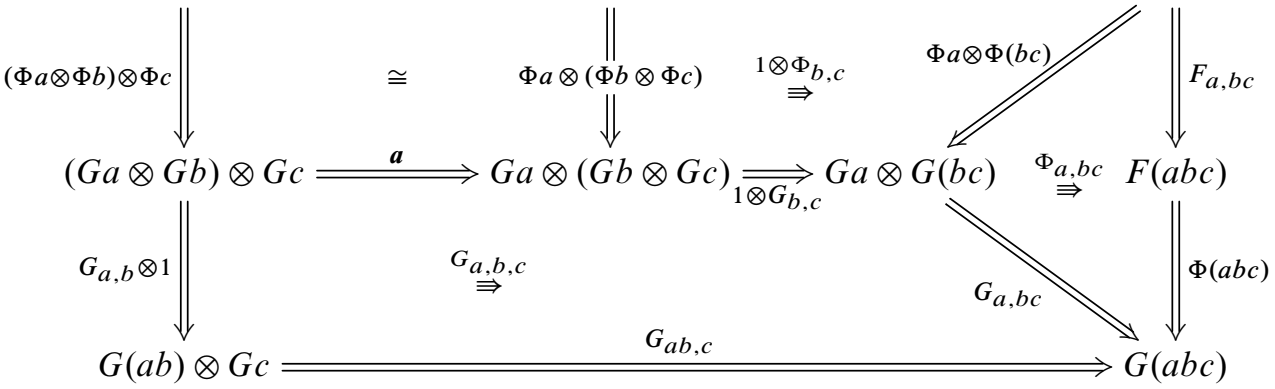

(CR5) For any morphism of $I, i \stackrel{a}{\longrightarrow} j$, the following two pasting equalities hold:
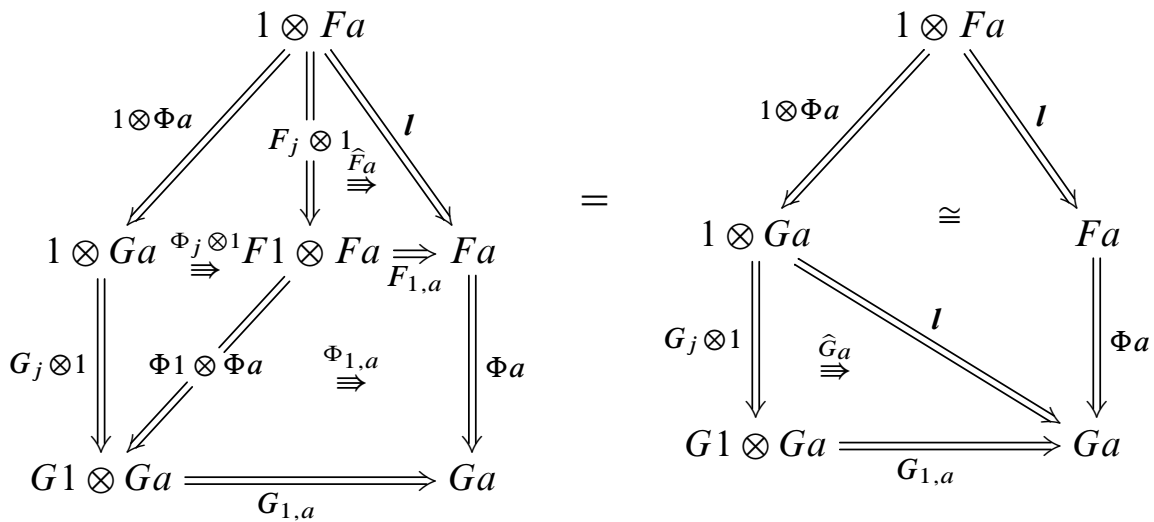

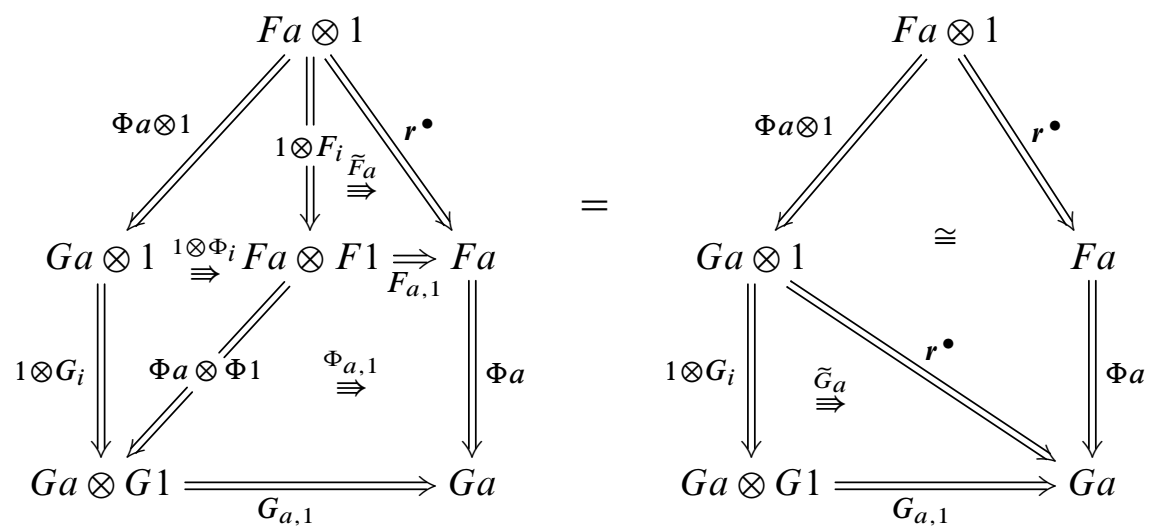

(CR6) For any object $i$ and each two composable arrows $i \stackrel{b}{\longrightarrow} j \stackrel{a}{\longrightarrow} k$ of $I$, the diagrams of 3-cells below commute:

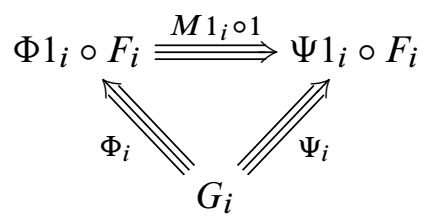

and

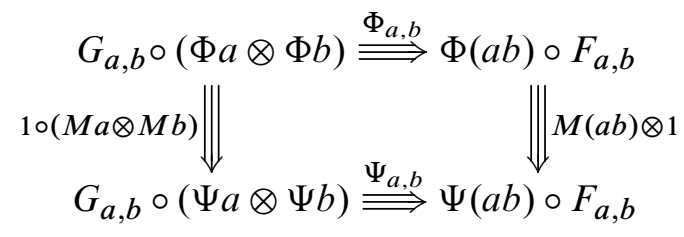

\section{A.1 Proof of Lemma 2.3}

(i) The homomorphism $H_{*}$ is defined as follows: it carries a lax functor $F: I \rightarrow \mathcal{T}$ to the lax functor $H_{*} F: I \rightarrow \mathcal{T}^{\prime}$, which is defined on objects $i$ of $I$ by $\left(H_{*} F\right) i=H F i$, and is defined on arrows $a: i \rightarrow j$ by $\left(H_{*} F\right) a=H F a: H F i \rightarrow H F j$. The 2-cell $\left(H_{*} F\right)_{a, b}:\left(H_{*} F\right) a \otimes\left(H_{*} F\right) b \Rightarrow\left(H_{*} F\right)(a b)$, for each pair of composable arrows $i \stackrel{b}{\longrightarrow} j \stackrel{a}{\longrightarrow} k$, is the composition

$$
H F a \otimes H F b \stackrel{\chi}{\Longrightarrow} H(F a \otimes F b) \stackrel{H F_{a, b}}{\Longrightarrow} H F(a b) .
$$

For each object $i$, the $2-$ cell $\left(H_{*} F\right)_{i}: 1_{\left(H_{*} F\right) i} \Rightarrow\left(H_{*} F\right) 1_{i}$ is the composite of

$$
1_{H F i} \stackrel{\iota}{\Longrightarrow} H 1_{F i} \stackrel{H F_{i}}{\Longrightarrow} H F 1_{i} \text {. }
$$


The structure 3-cell of $H_{*} F: I \rightarrow \mathcal{T}^{\prime}$ associated to any three composable arrows $i \stackrel{c}{\longrightarrow} j \stackrel{b}{\longrightarrow} k \stackrel{a}{\longrightarrow} l$ is that obtained by pasting the diagram

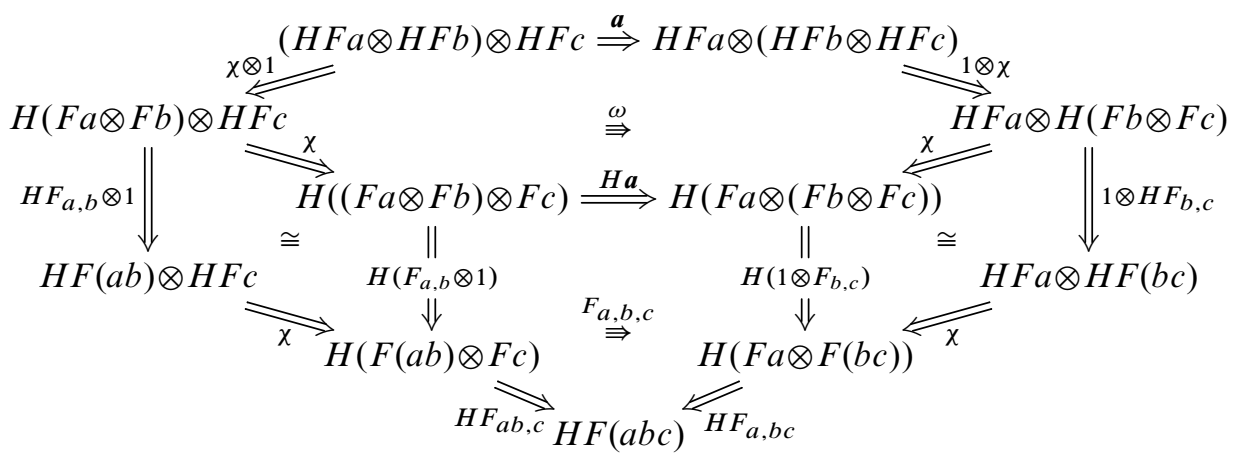

whereas the structure 3-cells of the lax functor $H_{*} F$ attached to an arrow $a: i \rightarrow j$ of the category $I$ are respectively those obtained by pasting the diagrams below:
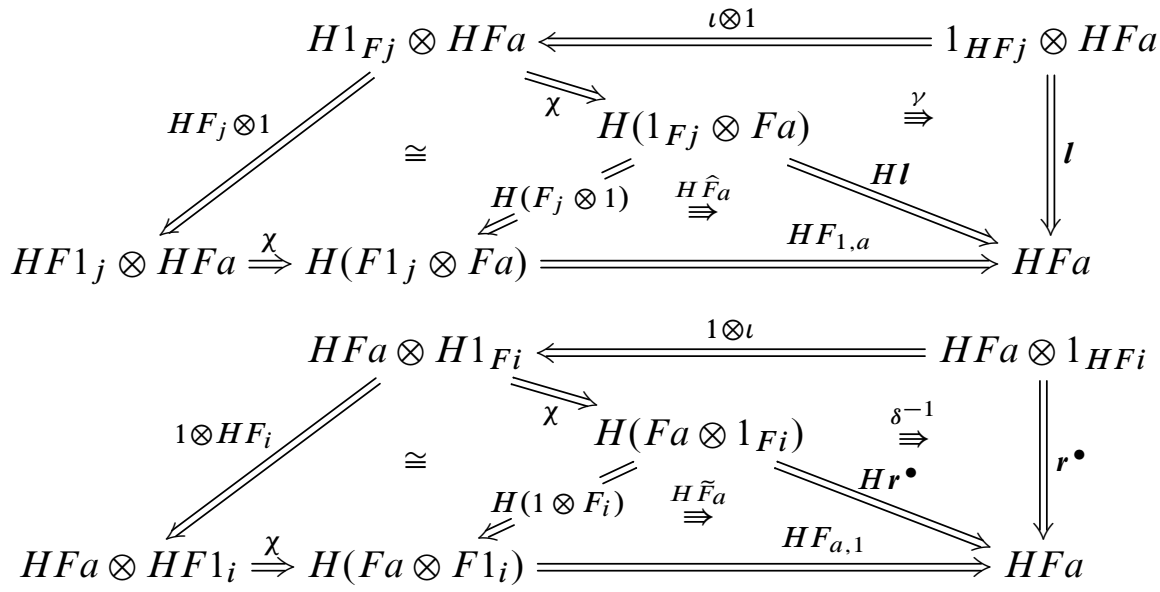

If $\Phi: F \Rightarrow G$ is any 1 -cell in the bicategory $\mathbb{L} a x(I, \mathcal{T})$, then $H_{*} \Phi: H_{*} F \Rightarrow H_{*} G$ is the 1-cell in $\operatorname{Lax}\left(I, \mathcal{T}^{\prime}\right)$ whose component at an arrow $a: i \rightarrow j$ of $I$ is the 2-cell of $\mathcal{T}^{\prime}$ defined by $\left(H_{*} \Phi\right) a=H \Phi a: H F a \Rightarrow H G a$. For any pair of composable arrows $i \stackrel{b}{\longrightarrow} j \stackrel{a}{\longrightarrow} k$ and any object $i$ of $I$, the corresponding structure 3-cells (3) and (4), $\left(H_{*} \Phi\right)_{a, b}$ and $\left(H_{*} \Phi\right)_{i}$, are respectively given by pasting in

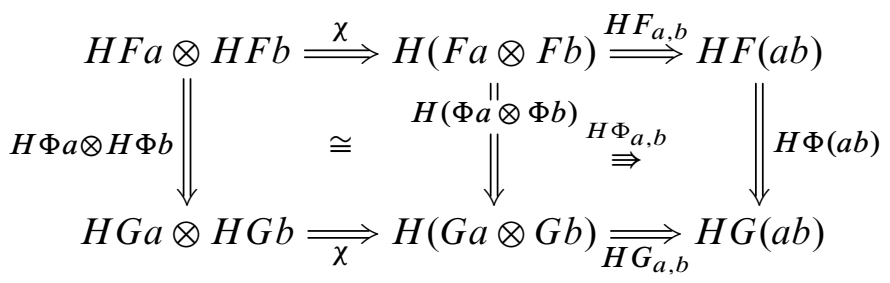


and

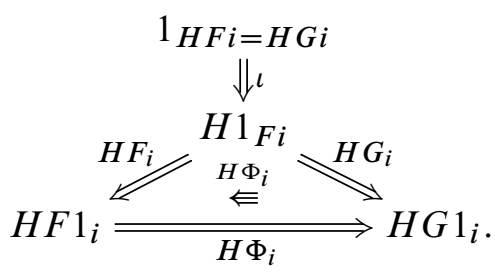

And a 2-cell $M: \Phi \Rightarrow \Psi$ of $\operatorname{Lax}(I, \mathcal{T})$ is applied by the homomorphism $H_{*}$ to the 2cell $H_{*} M: H_{*} \Phi \Rightarrow H_{*} \Psi$ of $\operatorname{Lax}\left(I, \mathcal{T}^{\prime}\right)$, such that $\left(H_{*} M\right) a=H M a: H \Phi a \Rightarrow H \Psi a$ for any arrow $a: i \rightarrow j$ of the category $I$.

Finally, if $\Phi: F \Rightarrow G$ and $\Psi: G \Rightarrow H$ are any two composable 1-cells in $\mathbb{L} a x(I, \mathcal{T})$, and $F: I \rightarrow \mathcal{T}$ is any lax functor, then the constraints $\left(H_{*} \Psi\right) \circ\left(H_{*} \Phi\right) \cong H_{*}(\Psi \circ \Phi)$ and $1_{H_{*} F} \cong H_{*} 1_{F}$ are the structure isomorphisms $H \Psi a \circ H \Phi a \cong H(\Psi a \circ \Phi a)$ and $1_{H F a} \cong H 1_{F a}$, at any arrow $a: i \rightarrow j$ of $I$, of the homomorphism $H: \mathcal{T}(F i, F j) \rightarrow$ $\mathcal{T}^{\prime}(H F i, H F j)$, respectively.

If $\alpha: I \rightarrow J$ is a functor then, recalling the definition of $\alpha^{*}$ given at the beginning of Section 2.2 , one easily checks the equality $H_{*} \alpha^{*}=\alpha^{*} H_{*}$.

(ii) For any lax functor $F: I \rightarrow \mathcal{T}$, the 2 -cell attached by

$$
m=m_{F}: H_{*}^{\prime}\left(H_{*} F\right) \Rightarrow\left(H^{\prime} H\right)_{*} F
$$

at any arrow $a: i \rightarrow j$ of $I$ is the identity, that is, $m a=1_{H^{\prime} H F a}$. For any pair of composable arrows $i \stackrel{b}{\longrightarrow} j \stackrel{a}{\longrightarrow} k$ and any object $i$ of $I$, the corresponding invertible structure 3-cells (3) and (4),

$$
\begin{aligned}
l m_{a, b}:\left(H_{*}^{\prime}\left(H_{*} F\right)\right)_{a, b} \circ(m a \otimes m b) & \Rightarrow m(a b) \circ\left(\left(H^{\prime} H\right)_{*} F\right)_{a, b}, \\
m_{i}: m 1_{i} \circ\left(H_{*}^{\prime}\left(H_{*} F\right)\right)_{i} & \Rightarrow\left(\left(H^{\prime} H\right)_{*} F\right)_{i},
\end{aligned}
$$

are, respectively, given by pasting in the diagrams below:

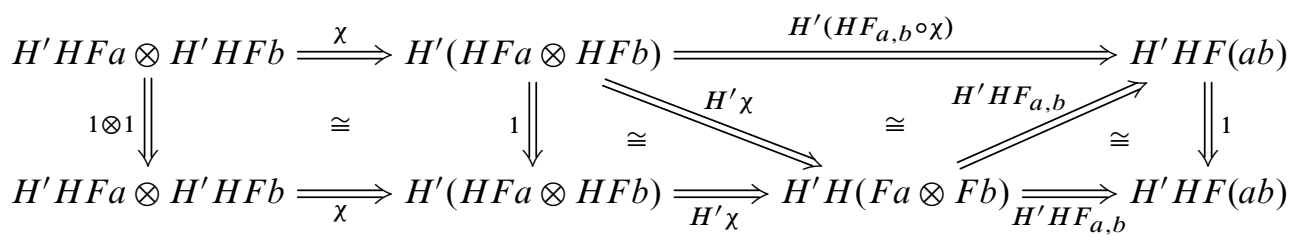


and

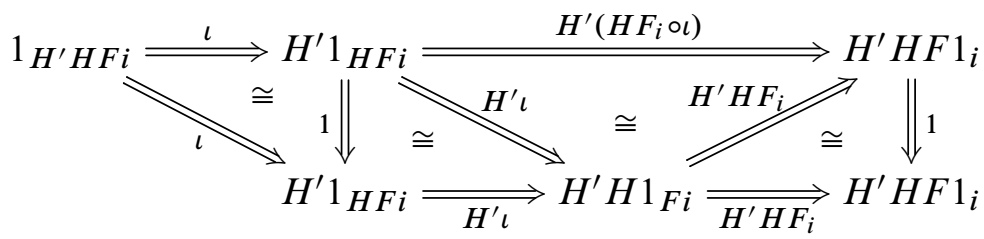

If $\Phi: F \Rightarrow G$ is any $1-$ cell in $\operatorname{Lax}(I, \mathcal{T})$, then we have that the invertible naturality 2-cell $m_{\Phi}: m_{G} \circ\left(H_{*}^{\prime}\left(H_{*} \Phi\right)\right) \Rightarrow\left(H^{\prime} H\right)_{*} \Phi \circ m_{F}$, at any arrow $a: i \rightarrow j$ of $I$, is the canonical isomorphism $1 \circ H^{\prime} H \Phi a \cong H^{\prime} H \Phi a \circ 1$ in $\mathcal{T}^{\prime \prime}\left(H^{\prime} H F i, H^{\prime} H F j\right)$.

For a functor $\alpha: I \rightarrow J$, it is easy to see that $m \alpha^{*}=\alpha^{*} m$.

(iii) For any lax functor $F: I \rightarrow \mathcal{T}$, the 2 -cell attached by

$$
m=m_{F}:\left(1_{\mathcal{T}}\right)_{*} F \Rightarrow F
$$

at any arrow $a: i \rightarrow j$ of $I$ is the identity, that is, $m a=1_{F a}$. For any pair of composable arrows $i \stackrel{b}{\longrightarrow} j \stackrel{a}{\longrightarrow} k$ and any object $i$ of $I$, the corresponding invertible structure 3-cells (3) and (4),

$$
m_{a, b}: F_{a, b} \circ(m a \otimes m b) \Rightarrow m(a b) \circ\left(\left(1_{\mathcal{T}}\right)_{*} F\right)_{a, b}, \quad m_{i}: m 1_{i} \circ\left(\left(1_{\mathcal{T}}\right)_{*} F\right)_{i} \Rightarrow F_{i},
$$

are, respectively, the canonical isomorphisms in the diagrams below:
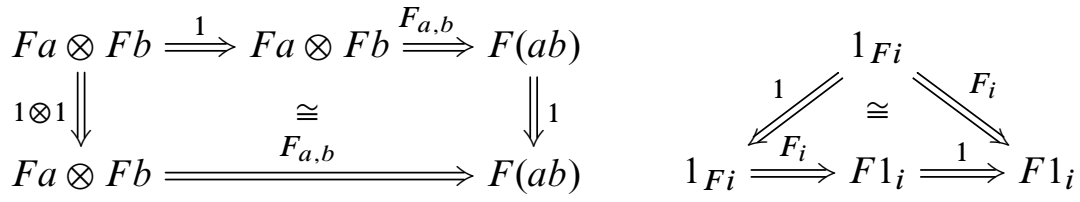

If $\Phi: F \Rightarrow G$ any $1-$ cell in $\operatorname{Lax}(I, \mathcal{T})$, then we have that the invertible naturality 2-cell $\left.m_{\Phi}: m_{G} \circ\left(\left(1_{\mathcal{T}}\right)_{*} \Phi\right)\right) \Rightarrow \Phi \circ m_{F}$, at any arrow $a: i \rightarrow j$ of $I$, is provided by the canonical isomorphism $1 \circ \Phi a \cong \Phi a \circ 1$ in $\mathcal{T}(F i, F j)$.

Again, for $\alpha: I \rightarrow J$ a functor, it is straightforward to check the equality $m \alpha^{*}=\alpha^{*} m$ holds.

\section{A.2 Proof of Lemma 2.4}

To describe the homomorphism $L$, we shall use the following useful construction: for any list $\left(t_{0}, \ldots, t_{p}\right)$ of objects in the tricategory $\mathcal{T}$, let

$$
\otimes^{\text {or: }} \mathcal{T}\left(t_{p-1}, t_{p}\right) \times \mathcal{T}\left(t_{p-2}, t_{p-1}\right) \times \cdots \times \mathcal{T}\left(t_{0}, t_{1}\right) \longrightarrow \mathcal{T}\left(t_{0}, t_{p}\right)
$$


denote the homomorphism recursively defined as the composite

$$
\mathcal{T}\left(t_{p-1}, t_{p}\right) \times \prod_{i=1}^{p-1} \mathcal{T}\left(t_{i-1}, t_{i}\right) \stackrel{1 \times \otimes}{\longrightarrow} \mathcal{T}\left(t_{p-1}, t_{p}\right) \times \mathcal{T}\left(t_{0}, t_{p-1}\right) \stackrel{\otimes}{\longrightarrow} \mathcal{T}\left(t_{0}, t_{p}\right) .
$$

That is, $\otimes^{\text {or }}$ is the homomorphism obtained by iterating composition in the tricategory, which acts on 0 -cells, $1-$ cells and 2 -cells of the product bicategory $\prod_{i=1}^{p} \mathcal{T}\left(t_{i-1}, t_{i}\right)$ by the recursive formula

$$
\otimes^{\text {or }}\left(x_{p}, \ldots, x_{1}\right)= \begin{cases}x_{1} & \text { if } p=1, \\ x_{p} \otimes\left(\otimes^{\text {or }}\left(x_{p-1}, \ldots, x_{1}\right)\right) & \text { if } p \geq 2 .\end{cases}
$$

The definition of $L$ on 0 -cells The homomorphism $L$ takes a lax functor of the graph in the tricategory, say $f: \mathcal{G} \rightarrow \mathcal{T}$, to the unitary homomorphic lax functor from the free category

$$
L(f)=F: I \rightarrow \mathcal{T},
$$

such that $F i=f i$, for any vertex $i$ of $\mathcal{G}$ (= objects of $I$ ), and associates to strings

$$
a: a(0) \stackrel{a_{1}}{\rightarrow} \cdots \stackrel{a_{p}}{\rightarrow} a(p)
$$

in $\mathcal{G}$ the 1 -cells $F a=\otimes^{\text {or }}\left(f a_{p}, \ldots, f a_{1}\right): f a(0) \rightarrow f a(p)$. The structure 2-cells $F_{a, b}: F a \otimes F b \Rightarrow F(a b)$, for any pair of strings in the graph, $a=a_{p} \cdots a_{1}$ as above and $b=b_{q} \cdots b_{1}$ with $b(q)=a(0)$, are canonically obtained from the associativity constraint in the tricategory: First by taking $F_{a_{1}, b}=1_{F\left(a_{1} b\right)}$ and then, recursively for $p>1$, defining $F_{a, b}$ as the composite

$$
F_{a, b}: F a \otimes F b \stackrel{a}{\Longrightarrow} F a_{p} \otimes\left(F a^{\prime} \otimes F b\right) \stackrel{1 \otimes F_{a^{\prime}, b}}{\Longrightarrow} F(a b),
$$

where $a^{\prime}=a_{p-1} \cdots a_{1}$ (whence $F a=F a_{p} \otimes F a^{\prime}$ ). And the structure 3-cells $F_{a, b, c}$, for any three strings in the graph $a, b$ and $c$ as above with $a(0)=b(q)$ and $b(0)=c(r)$, are the unique isomorphisms constructed from the tricategory coherence 3-cells $\pi$. For a particular construction of these isomorphisms, we can first take each $F_{a_{1}, b, c}$ to be the canonical isomorphism $F_{a_{1}, b, c}$ given by

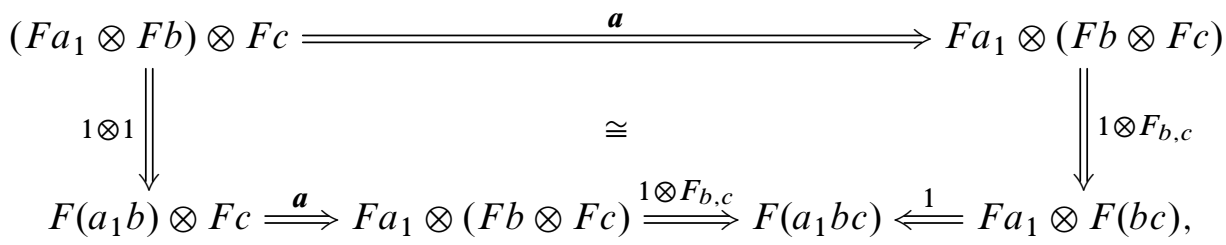

and then, recursively for $p>1$, take $F_{a, b, c}$ to be the 3-cell canonically obtained from $F_{a_{p-1} \cdots a_{1}, b, c}$ by pasting the diagram below, where, as above, we write $a^{\prime}$ 
for $a_{p-1} \cdots a_{1}$ :

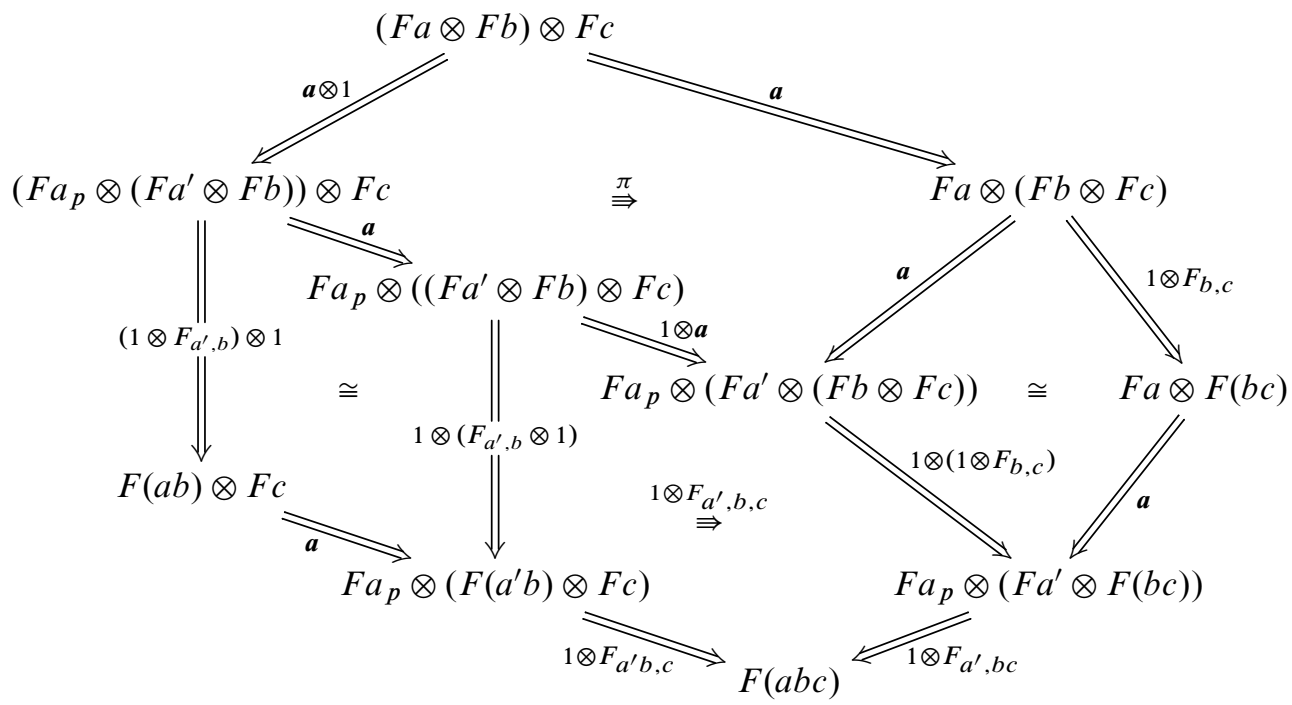

The conditions (CR1), (CR2) and (CR3) are verified thanks to Fact 1.1, since we are only using constraints 2-cells and 3-cells. Note that since all structure 2-cells $F_{a, b}$ are equivalences in the corresponding hom bicategories of $\mathcal{T}$ in which they lie, and all the structure 3-cells $F_{a, b, c}$ are invertible, the so defined unitary lax functor $F: I \rightarrow \mathcal{T}$ is actually a homomorphic one; that is, $L(f)=F \in \operatorname{Lax}_{\mathrm{uh}}(I, \mathcal{T}) \subseteq \mathbb{L a x}(I, \mathcal{T})$.

The definition of $L$ on 1-cells Any $1-$ cell $\phi: f \Rightarrow g$ of $\operatorname{Lax}(\mathcal{G}, \mathcal{T})$ is taken by $L$ to the $1-$ cell in $\operatorname{Lax}_{\mathrm{uh}}(I, \mathcal{T})$

$$
L(\phi)=\Phi: F \Rightarrow G
$$

consisting of the 2-cells in the tricategory $\Phi a=\otimes^{\text {or }}\left(\phi a_{p}, \ldots, \phi a_{1}\right): F a \Rightarrow G a$, attached to the strings of adjacent edges in the graph $a=a_{p} \cdots a_{1}$. The structure (actually invertible) 3-cells $\Phi_{a, b}$, for any pair of strings $a$ and $b$ in the graph with $b(q)=a(0)$ as above, are defined by induction on the length of $a$ as follows: Each $\Phi_{a_{1}, b}$ is the canonical isomorphism

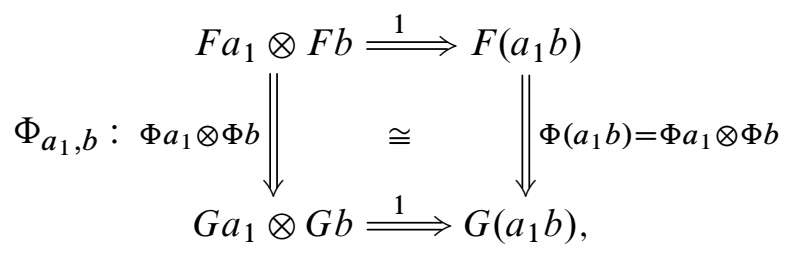


and, for $p>1$, each $\Phi_{a, b}$ is obtained from $\Phi_{a^{\prime}, b}$, where $a^{\prime}=a_{p-1} \cdots a_{1}$, by pasting

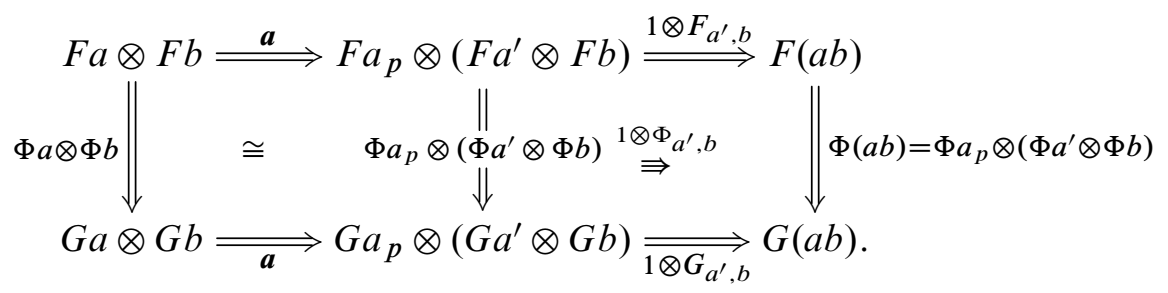

Again, Fact 1.1 ensures that conditions (CR4) and (CR5) are satisfied. Note that, since all structure 3-cells $\Phi_{a, b}$ are invertible, the thus defined unitary 1-cell $L(\phi)=\Phi$ of $\operatorname{Lax}_{\mathrm{u}}(I, \mathcal{T})$ is actually a $1-$ cell of $\operatorname{Lax}_{\mathrm{uh}}(I, \mathcal{T})$.

The definition of $\boldsymbol{L}$ on 2-cells For $\phi, \psi: f \Rightarrow g$ and any two 1-cells in $\operatorname{Lax}(\mathcal{G}, \mathcal{T})$, the homomorphism $L$ on a 2 -cell $m: \phi \Rightarrow \psi$ gives the 2 -cell of $\operatorname{Lax}_{\mathrm{uh}}(I, \mathcal{T})$

$$
L(m)=M: \Phi \Rightarrow \Psi
$$

consisting of the 3-cells in the tricategory $M a=\otimes{ }^{\text {or }}\left(m a_{p}, \ldots, m a_{1}\right): \Phi a \Rightarrow \Psi a$ for the strings $a=a_{p} \cdots a_{1}$ of adjacent edges in the graph $\mathcal{G}$.

The structure constraints of $\boldsymbol{L}$ If $\phi: f \Rightarrow g$ and $\psi: g \Rightarrow h$ are $1-$ cells in $\operatorname{Lax}(\mathcal{G}, \mathcal{T})$, then the structure isomorphism in $\operatorname{Lax}_{\mathrm{u}}(I, \mathcal{T})$

$$
L_{\psi, \phi}: L(\psi) \circ L(\phi) \cong L(\psi \circ \phi)
$$

at each string $a=a_{p} \cdots a_{1}$ as above, is recursively defined as the identity 3-cell on $\psi a_{1} \circ \phi a_{1}$ if $p=1$, while, for $p>1, L_{\psi, \phi} a$ : $L(\psi) a \circ L(\phi) a \Rightarrow L(\psi \circ \phi) a$ is obtained from $L_{\psi, \phi} a^{\prime}$, where $a^{\prime}=a_{p-1} \cdots a_{1}$, as the composite

$$
\begin{aligned}
L(\psi) a \circ L(\phi) a & =\left(\psi a_{p} \otimes L(\psi) a^{\prime}\right) \circ\left(\phi a_{p} \otimes L(\phi) a^{\prime}\right) \\
& \cong\left(\psi a_{p} \circ \phi a_{p}\right) \otimes\left(L(\psi) a^{\prime} \circ L(\phi) a^{\prime}\right) \\
& \stackrel{L_{\psi, \phi} a^{\prime}}{\Rightarrow}\left(\psi a_{p} \circ \phi a_{p}\right) \otimes L(\psi \circ \phi) a^{\prime}=L(\psi \circ \phi) a .
\end{aligned}
$$

Similarly, the structure isomorphism $L_{f}: 1_{L(f)} \cong L\left(1_{f}\right)$ consists of the 3-cells $L_{f} a: 1_{L(f) a} \Rightarrow L\left(1_{f}\right) a$, where $L_{f} a_{1}=1: 1_{f a_{1}} \Rightarrow 1_{f a_{1}}$ and, for $p>1, L_{f} a$ is recursively obtained from $L_{f} a^{\prime}, a^{\prime}=a_{p-1} \cdots a_{1}$, as the composite

$$
1_{L(f) a}=1_{f a_{p} \otimes L(f) a^{\prime}} \cong 1_{f a_{p}} \otimes 1_{L(f) a^{\prime}} \stackrel{1 \otimes L_{f} a^{\prime}}{\Rightarrow} 1_{f a_{p}} \otimes L\left(1_{f}\right) a^{\prime}=L\left(1_{f}\right) a .
$$

This completes the description of the homomorphism $L$. 
The definition of the lax transformation $\mathbf{v}$ The component of this lax transformation at a lax functor $F: I \rightarrow \mathcal{T}, \mathrm{v}=\mathrm{v}(F): L R(F) \Rightarrow F$, is defined on identities by

$$
\mathrm{v}_{i}=F_{i}: 1_{F i} \Rightarrow F 1_{i},
$$

for any vertex $i$ of $\mathcal{G}$, and it associates to each string of adjacent edges in the graph $a=a_{p} \cdots a_{1}$ the $2-$ cell

$$
\mathrm{v} a: \otimes^{\mathrm{or}}\left(F a_{p}, \ldots, F a_{1}\right) \Rightarrow F a,
$$

which is given by taking $\mathrm{v} a_{1}=1_{F a_{1}}$ if $p=1$, and then, recursively for $p>1$, by taking $\mathrm{v} a$ as the composite

$$
\mathrm{v} a: \otimes^{\mathrm{or}}\left(F a_{p}, \ldots, F a_{1}\right) \stackrel{1 \otimes \mathrm{v} a^{\prime}}{\longrightarrow} F a_{p} \otimes F a^{\prime} \stackrel{F_{a_{p}, a^{\prime}}}{\longrightarrow} F a
$$

where $a^{\prime}=a_{p-1} \cdots a_{1}$.

The structure 3-cell

$$
\mathrm{v}_{a, b}: F_{a, b} \circ(\mathrm{v} a \otimes \mathrm{v} b) \Rightarrow \mathrm{v}(a b) \circ L R(F)_{a, b},
$$

for any pair of composable morphisms in $I$, is defined as follows: when $a=1_{j}$ or $b=1_{i}$ are identities, then $\mathrm{v}_{1_{j}, b}$ and $\mathrm{v}_{a, 1_{i}}$ are respectively given by pasting the diagrams

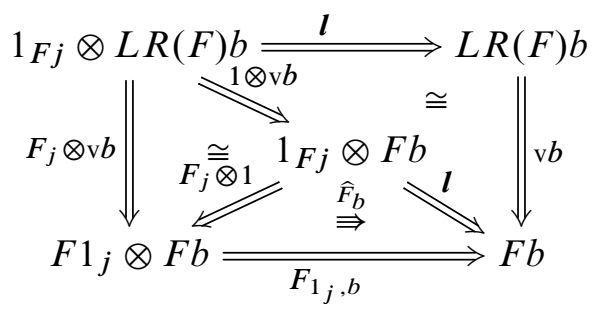

and

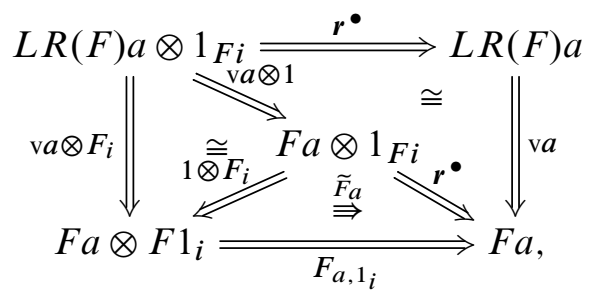


and, for strings $a$ and $b$ in the graph with $b(q)=a(0), \mathrm{v}_{a, b}$ is defined by induction on the length of $a$ by taking $\mathrm{v}_{a_{1}, b}$ to be the canonical isomorphism

$$
\begin{aligned}
& F a_{1} \otimes L R(F) b \stackrel{1}{\Longrightarrow} \operatorname{LR}(F)\left(a_{1} b\right) \\
& 1 \otimes \mathrm{v} b \| \cong \underset{\Downarrow \mathrm{v}\left(a_{1} b\right)=F_{a_{1}, b} \circ(1 \otimes \mathrm{v} b)}{ } \\
& F a_{1} \otimes F b \underset{F_{a_{1}, b}}{\Longrightarrow} F\left(a_{1} b\right)
\end{aligned}
$$

and then, for $p>1, \mathrm{v}_{a, b}$ is obtained from $\mathrm{v}_{a^{\prime}, b}$, where $a^{\prime}=a_{p-1} \cdots a_{1}$, by pasting

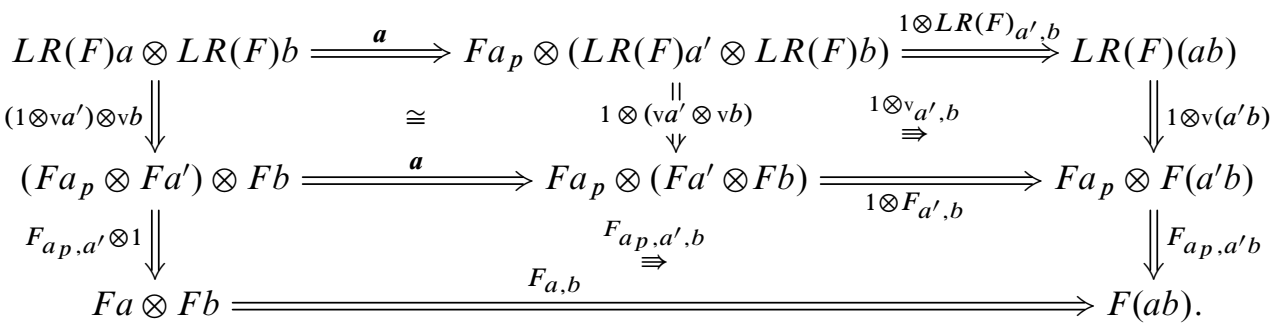

And the structure 3 -cell

$$
\mathrm{v}_{i}: \mathrm{v}_{i} \circ L R(F)_{i} \Rightarrow F_{i},
$$

for any vertex $i$ of the graph, is the canonical isomorphism $F_{i} \circ 1 \cong F_{i}$. Conditions (CR4) and (CR5) are verified by using conditions (CR1), (CR2) and (CR3) for $F$ and Facts 1.1, 1.2 and 1.3.

The naturality component of $\mathrm{v}$ at a $1-$ cell $\Phi: F \Rightarrow G$ in $\mathbb{L a x}_{\mathrm{u}}(I, \mathcal{T})$,

$$
\mathrm{v}_{\Phi}: \mathrm{v}(G) \circ L R(\Phi) \Rightarrow \Phi \circ \mathrm{v}(F)
$$

is given on identities by

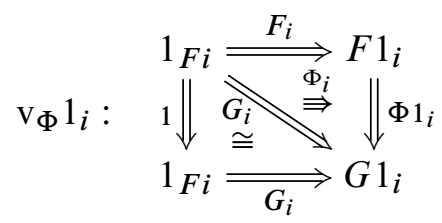

and it is recursively defined at each string in the graph $a=a_{p} \cdots a_{1}$, by the 3 -cells $\mathrm{v}_{\Phi} a$, where

$$
\begin{aligned}
F a_{1} & \stackrel{1}{\Longrightarrow} F a_{1} \\
\mathrm{v}_{\Phi} a_{1}: \Phi a_{1} \Downarrow & \cong \Downarrow \Phi a_{1} \\
G a_{1} & \stackrel{1}{\Longrightarrow} G a_{1}
\end{aligned}
$$


and then, when $p>1, \mathrm{v}_{\Phi} a$ is obtained from $\mathrm{v}_{\Phi} a^{\prime}$, where $a^{\prime}=a_{p-1} \cdots a_{1}$, by pasting

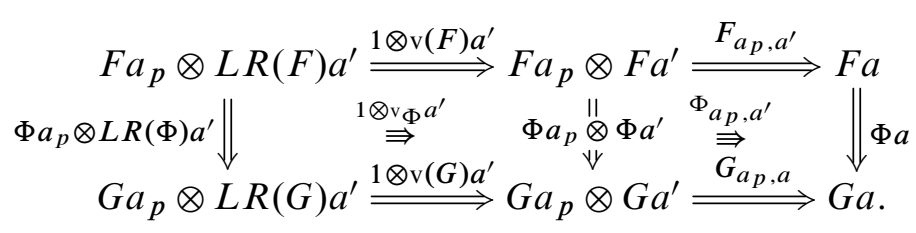

Condition (CR6) for this 2-cell is verified using Conditions (CR4) and (CR5) for the $1-$ cell $\Phi$, together with Facts 1.1, 1.2 and 1.3.

We are now ready to complete the proof. That the equalities $R L=1, \mathrm{v} L=1$ and $R \mathrm{v}=1$ hold only requires a straightforward verification, and then (i) follows. Moreover, (ii) has already been shown by construction of the homomorphism $L$.

(iii) Suppose that $F: I \rightarrow \mathcal{T}$ is any homomorphic lax functor. This means that all structure 2-cells $F_{a, b}$ and $F_{i}$ are equivalences, and 3-cells $F_{a, b, c}, \widehat{F}_{a}$ and $\widetilde{F}_{a}$ are isomorphisms in the hom bicategories of $\mathcal{T}$ in which they lie. Then, directly from the construction given, it easily follows that all the 2-cells $\mathrm{v}(F) a$ in (2) are equivalences in the corresponding hom bicategories and that all the 3-cells $\mathrm{v}(F)_{a, b}$ in (3) and $\mathrm{v}_{i}$ in (4) are invertible. Hence, each $\mathrm{v}(F): L R(F) \Rightarrow F$, for $F: I \rightarrow \mathcal{T}$ any homomorphic lax functor, is an equivalence in the bicategory $\operatorname{Lax}_{\mathrm{h}}(I, \mathcal{T})$. Moreover, if $\Phi: F \Rightarrow G$ is any 1 -cell in $\operatorname{Lax}_{\mathrm{h}}(I, \mathcal{T})$, so that every 3-cell $\Phi_{a, b}$ and $\Phi_{i}$ is an isomorphism, then we see that the component (5) of $\mathrm{v}$ at $\Phi$ consists only of invertible 3-cells $\mathrm{v}_{\Phi} a$, whence $\mathrm{v}_{\Phi}$ is invertible itself. Therefore, when $\mathrm{v}$ is restricted to $\operatorname{Lax}_{\mathrm{h}}(I, \mathcal{T})$, it actually gives a pseudoequivalence between $L R$ and 1 , the identity homomorphism on the bicategory $\operatorname{Lax}_{\mathrm{h}}(I, \mathcal{T})$. The claimed biadjoint biequivalence (7) is now an easy consequence of all the parts already proved. Finally, it is clear that the biadjoint biequivalence (7) gives by restriction the biadjoint biequivalence (8).

Acknowledgements The authors are much indebted to the anonymous referee, whose useful observations greatly improved our exposition. This work has been supported by DGI of Spain, Project MTM2011-22554 and for the second author also by Ministerio de Educación, Cultura y Deportes of Spain, FPU grant AP2010-3521. The authors would like to express their gratitude to Eduardo Pareja Tobes for the useful discussions we maintained during the realization of this paper.

\section{References}

[1] J C Baez, The homotopy hypothesis Available at http://math.ucr.edu/home/ baez/homotopy 
[2] J C Baez, M Neuchl, Higher-dimensional algebra, I: Braided monoidal 2-categories, Adv. Math. 121 (1996) 196-244 MR1402727

[3] C Balteanu, Z Fiedorowicz, R Schwänzl, R Vogt, Iterated monoidal categories, Adv. Math. 176 (2003) 277-349 MR1982884

[4] J Bénabou, Introduction to bicategories, from: "Reports of the Midwest Category Seminar", Springer (1967) 1-77 MR0220789

[5] C Berger, Double loop spaces, braided monoidal categories and algebraic 3-type of space, from: "Higher homotopy structures in topology and mathematical physics", Contemp. Math. 227, Amer. Math. Soc. (1999) 49-66 MR1665460

[6] A K Bousfield, D M Kan, Homotopy limits, completions and localizations, Lecture Notes in Mathematics 304, Springer (1972) MR0365573

[7] M Bullejos, A M Cegarra, On the geometry of 2-categories and their classifying spaces, K-Theory 29 (2003) 211-229 MR2028502

[8] M Bullejos, A M Cegarra, Classifying spaces for monoidal categories through geometric nerves, Canad. Math. Bull. 47 (2004) 321-331 MR2072592

[9] P Carrasco, A M Cegarra, (Braided) tensor structures on homotopy groupoids and nerves of (braided) categorical groups, Comm. Algebra 24 (1996) 3995-4058 MR1414569

[10] P Carrasco, A M Cegarra, Schreier theory for central extensions of categorical groups, Comm. Algebra 24 (1996) 4059-4112 MR1414570

[11] P Carrasco, A M Cegarra, A R Garzón, Nerves and classifying spaces for bicategories, Algebr. Geom. Topol. 10 (2010) 219-274 MR2602835

[12] P Carrasco, A M Cegarra, A R Garzón, Classifying spaces for braided monoidal categories and lax diagrams of bicategories, Adv. Math. 226 (2011) 419-483 MR2735766

[13] A M Cegarra, A R Garzón, Homotopy classification of categorical torsors, Appl. Categ. Structures 9 (2001) 465-496 MR1865612

[14] A M Cegarra, E Khmaladze, Homotopy classification of graded Picard categories, Adv. Math. 213 (2007) 644-686 MR2332605

[15] E Cheng, N Gurski, The periodic table of n-categories for low dimensions II: Degenerate tricategories arXiv:0706.2307

[16] J W Duskin, Simplicial matrices and the nerves of weak $n$-categories. I: Nerves of bicategories, Theory Appl. Categ. 9 (2002) 198-308 MR1897816

[17] Z Fiedorowicz, The symmetric bar construction (1998) Available at http:// www.math. osu. edu/ fiedorow/symbar.ps.gz

[18] R Garner, N Gurski, The low-dimensional structures formed by tricategories, Math. Proc. Cambridge Philos. Soc. 146 (2009) 551-589 MR2496344 
[19] P G Goerss, J F Jardine, Simplicial homotopy theory, Progress in Mathematics 174, Birkhäuser, Basel (1999) MR1711612

[20] R Gordon, A J Power, R Street, Coherence for tricategories, Mem. Amer. Math. Soc. 117 (1995) vi+81 MR1261589

[21] A Grothendieck, Catégories fibrées et descente, from: "Séminaire de géométrie algébrique du Bois Marie 1966/67”, (P Berthelot, A Grothendieck, L Illusie, editors), Lecture Notes in Math. 224, Springer (1971) 145-194

[22] N Gurski, Nerves of bicategories as stratified simplicial sets, J. Pure Appl. Algebra 213 (2009) 927-946 MR2498786

[23] N Gurski, Loop spaces, and coherence for monoidal and braided monoidal bicategories, Adv. Math. 226 (2011) 4225-4265 MR2770448

[24] N Gurski, Biequivalences in tricategories, Theory Appl. Categ. 26 (2012) 349-384 MR2972968

[25] N Gurski, Coherence in three-dimensional category theory, Cambridge Tracts in Mathematics 201, Cambridge Univ. Press (2013) MR3076451

[26] K A Hardie, K H Kamps, R W Kieboom, A homotopy bigroupoid of a topological space, Appl. Categ. Structures 9 (2001) 311-327 MR1836257

[27] L Illusie, Complexe cotangent et déformations, II, Lecture Notes in Mathematics 283, Springer (1972) MR0491681

[28] J F Jardine, Supercoherence, J. Pure Appl. Algebra 75 (1991) 103-194 MR1138365

[29] A Joyal, R Street, Braided tensor categories, Adv. Math. 102 (1993) 20-78 MR1250465

[30] A Joyal, M Tierney, Algebraic homotopy types, Handwritten lecture notes (1984)

[31] M M Kapranov, V A Voevodsky, 2-categories and Zamolodchikov tetrahedra equations, from: "Algebraic groups and their generalizations: quantum and infinitedimensional methods", (W J Haboush, B J Parshall, editors), Proc. Sympos. Pure Math. 56, Amer. Math. Soc. (1994) 177-259 MR1278735

[32] S Lack, Icons, Appl. Categ. Structures 18 (2010) 289-307 MR2640216

[33] S Lack, A Quillen model structure for Gray-categories, J. K-Theory 8 (2011) 183-221 MR2842929

[34] S Lack, S Paoli, 2-nerves for bicategories, K-Th. 38 (2008) 153-175 MR2366560

[35] O Leroy, Sur une notion de 3-catégorie adaptée à l'homotopie (1994)

[36] J P May, The spectra associated to permutative categories, Topology 17 (1978) 225228 MR508886

[37] D Quillen, Higher algebraic K-theory, I, from: "Algebraic $K$-theory, I: Higher $K$ theories”, Lecture Notes in Math. 341, Springer (1973) 85-147 MR0338129 
[38] G Segal, Classifying spaces and spectral sequences, Inst. Hautes Études Sci. Publ. Math. (1968) 105-112 MR0232393

[39] G Segal, Categories and cohomology theories, Topol. 13 (1974) 293-312 MR0353298

[40] C Simpson, Homotopy theory of higher categories, New Mathematical Monographs 19, Cambridge Univ. Press (2012) MR2883823

[41] J D Stasheff, Homotopy associativity of $H$-spaces, I, II, Trans. Amer. Math. Soc. 108 (1963) 293-312 MR0158400

[42] D Stevenson, The geometry of bundle gerbes, $\mathrm{PhD}$ thesis, University of Adelaide (2000) Available at http://arxiv.org/abs/math/0004117

[43] R Street, The algebra of oriented simplexes, J. Pure Appl. Algebra 49 (1987) 283-335 MR920944

[44] R Street, Categorical structures, from: "Handbook of algebra, Vol. 1", (M Hazewinkel, editor), Handb. Algebr. 1, Elsevier/North-Holland, Amsterdam (1996) 529-577 MR1421811

[45] R Street, Categorical and combinatorial aspects of descent theory, Appl. Categ. Structures 12 (2004) 537-576 MR2107402

[46] $\mathbf{Z}$ Tamsamani, Sur des notions de $n$-catégorie et $n$-groupoüde non strictes via des ensembles multi-simpliciaux, K-Theory 16 (1999) 51-99 MR1673923

[47] R W Thomason, Homotopy colimits in the category of small categories, Math. Proc. Cambridge Philos. Soc. 85 (1979) 91-109 MR510404

Department of Algebra, Faculty of Sciences

University of Granada, 18071, Granada, Spain

acegarra@ugr.es, baheredia@ugr.es

www.ugr.es/local/acegarra, www.ugr.es/local/baheredia

Received: 4 September 2013 Revised: 5 December 2013 